\title{
THE EFFECTS OF BOTULINUM TOXIN A (BTX-A) ON GAIT IN CHRONIC STROKE
}

By

Alison C. Novak

A thesis submitted to the Centre for Neuroscience Studies in conformity with the requirements for the degree of Master of Science

\author{
Queen's University \\ Kingston, Ontario, Canada \\ August 2007
}

copyright $\odot$ Alison C. Novak, 2007 


\begin{abstract}
Excessive muscle tone or stiffness secondary to stroke frequently involves the ankle plantarflexors and has been associated with decreased mobility and reduced function. Although becoming more common in clinical practice, the effectiveness of botulinum toxin A (BTX-A) injected in the ankle plantarflexors on gait biomechanics is not well established. The primary objective of this study was to describe the kinematic and kinetic changes that occur during walking following BTX-A treatment of the hypertonic ankle plantarflexors. As well, the study explored whether there were clinical characteristics uniquely associated with subjects that exhibited biomechanical improvement. The study was a single group, open label trial with repeated measures, including multiple baseline and three post-intervention time points. Seven chronic hemiparetic stroke subjects with ankle hypertonicity were included in the study. Full lower limb bilateral gait analysis provided joint kinematic and kinetic information throughout stance. As well, clinical measures of ankle range of motion and spasticity were assessed pre and post treatment. Data were analyzed using paired samples t-tests and repeated measures ANOVA with Least Significant Difference adjustment for posthoc analysis as necessary (significance level $\mathrm{p} \leq 0.05$ ). Of the kinematic variables, significant improvements in peak dorsiflexion and plantarflexion and the ankle angle at initial contact were found 10 weeks post-injection relative to baseline. No significant kinetic changes were detected, however 2 subjects showed improved positive work at the ankle post-injection and 5 subjects demonstrated increased positive work at the hip posttreatment. Although subjects were classified as "responders" or "non-responders" based
\end{abstract}


on clinical improvement observed 2 weeks post-injection, there was no observable association between those who responded clinically and those who demonstrated improved gait. The major findings suggest that BTX-A injection results in tone reduction and in some cases improves the biomechanical efficiency of gait. In cases where kinetic variables remained unchanged following treatment, perhaps the increased tone was not the limiting factor of reduced function. 


\section{ACKNOWLEDGEMENTS}

It was my privilege to be a student of Dr. Brenda Brouwer. I would like to extend my deepest sense of gratitude and appreciation for her constant guidance, advice, patience and encouragement throughout the course of this thesis.

I would also like to extend my thanks and appreciation to Dr. Stephen Bagg of St. Mary's of the Lake Hospital for his expertise, guidance, support and patient recruitment.

I would like to thank Allergan Inc. for financial support of this study.

I would like to extend my appreciation to all the volunteers who participated in this study and their families.

I would like to thank Dr. Brendan Joss and Leone Ploeg for all the help (and time) they have given to me throughout my data collection and processing.

I would like to thank all those people in the Motor Performance Lab, School of Rehabilitation Therapy and Centre for Neuroscience Studies who have helped over the past two years.

Lastly, I would like to extend a special thank you to my family for the constant love, support, motivation and patience they have given to me. 


\section{TABLE OF CONTENTS}

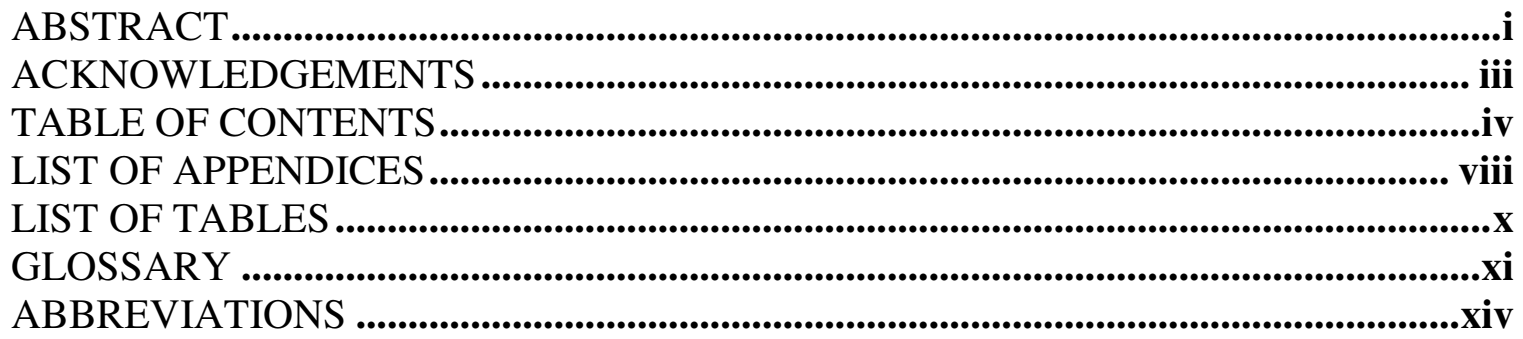

\section{CHAPTER 1}

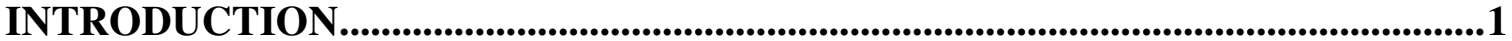

\section{CHAPTER 2}

LITERATURE REVIEW ...........................................................................................4

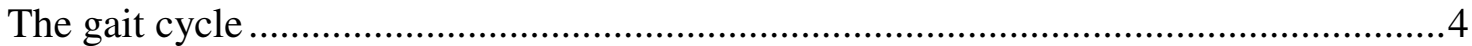

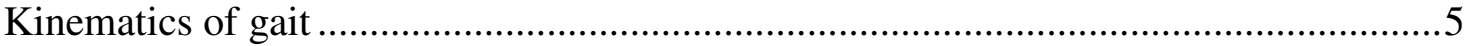

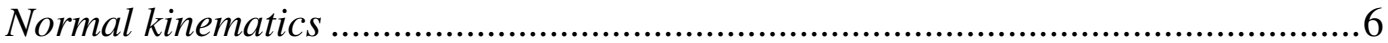

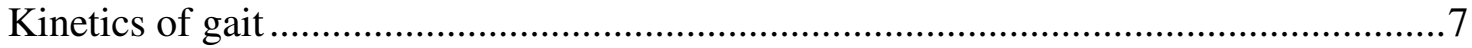

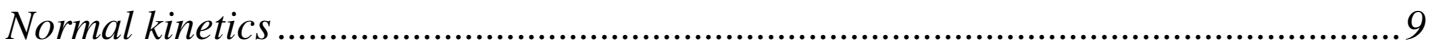

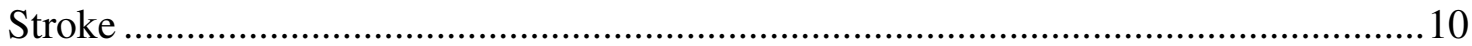

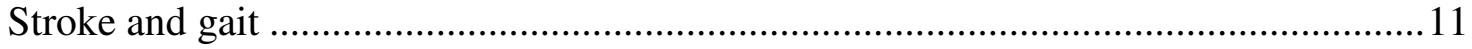

Temporal spatial variables and stroke ............................................................. 11

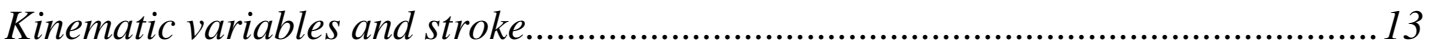

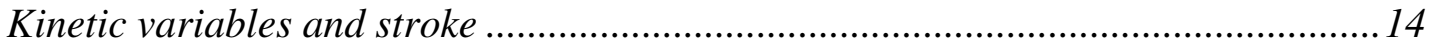

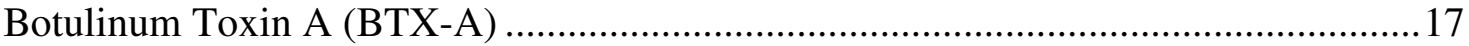




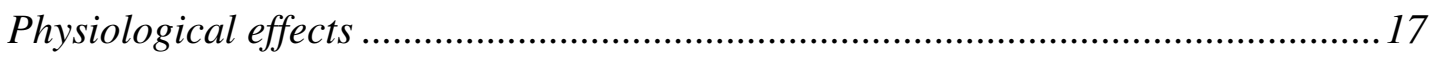

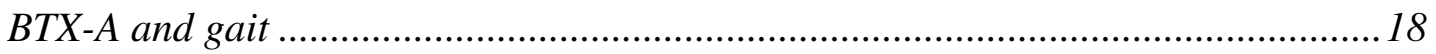

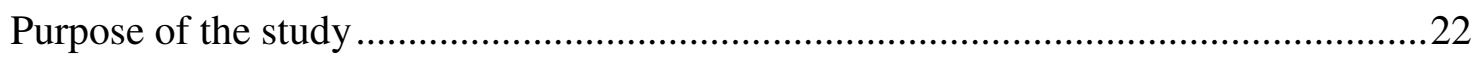

\section{CHAPTER 3}

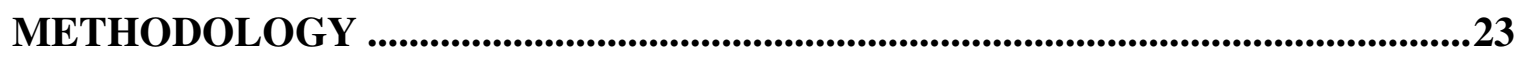

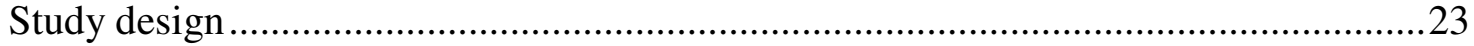

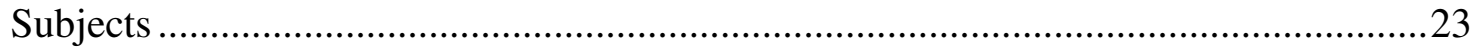

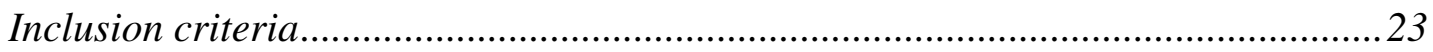

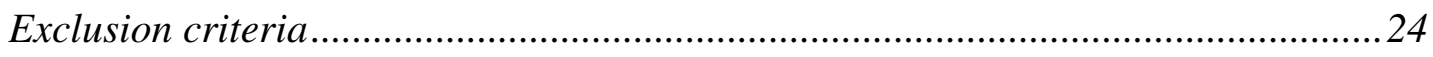

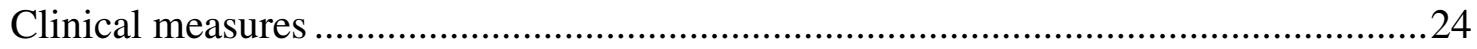

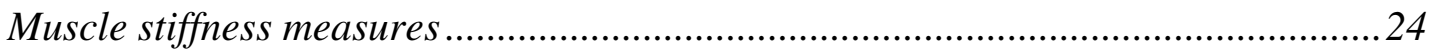

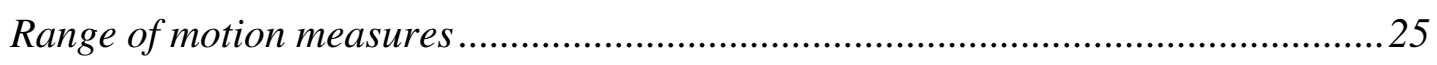

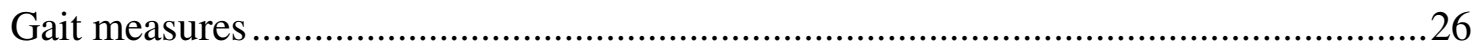

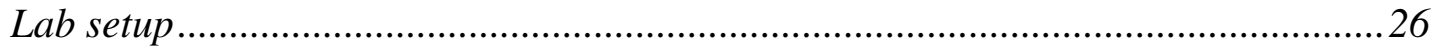

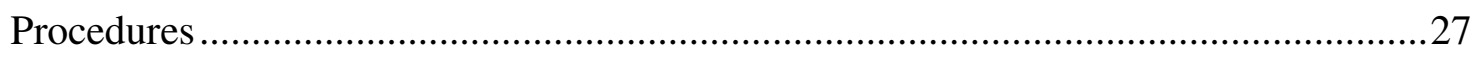

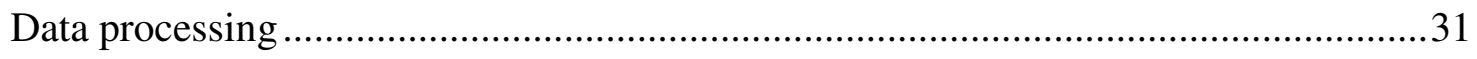

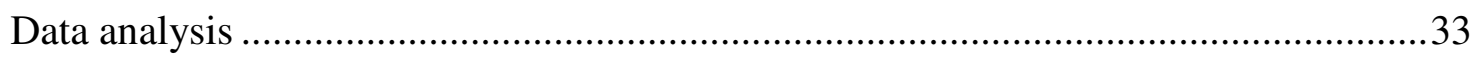

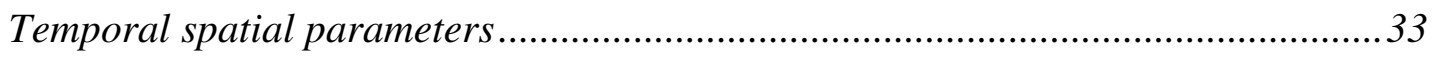

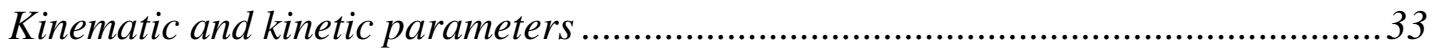

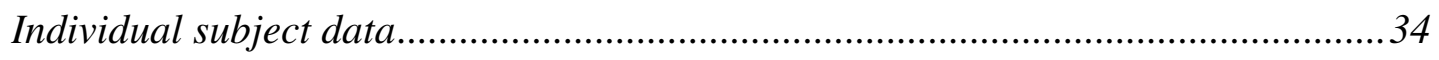

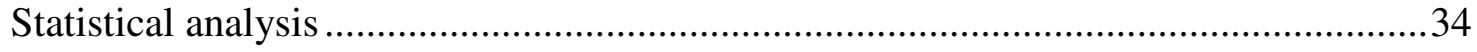




\section{CHAPTER 4}

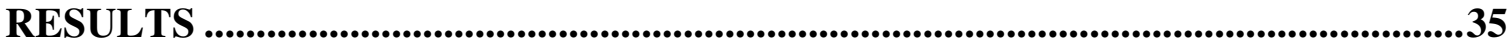

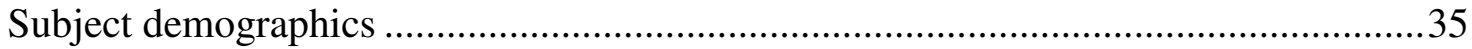

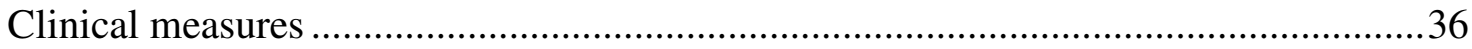

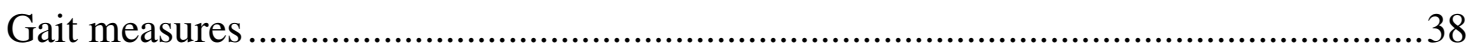

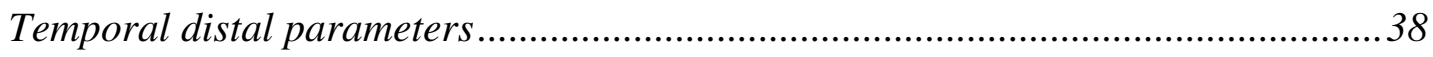

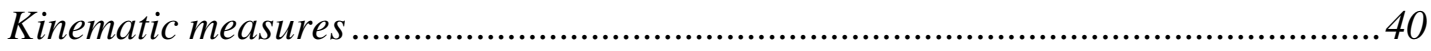

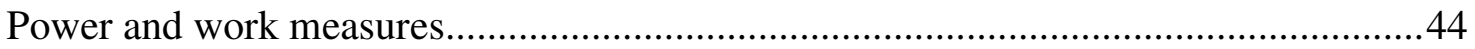

\section{CHAPTER 5}

DISCUSSION ..........................................................................................................................50

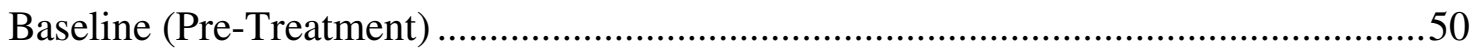

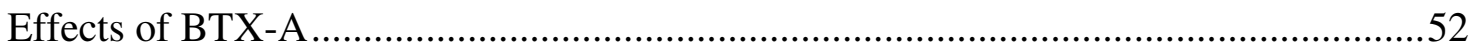

Temporal distal parameters ...............................................................................5

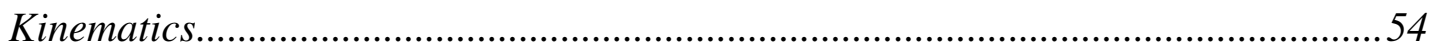

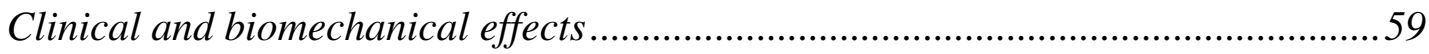

\section{CHAPTER 6}

CONCLUSIONS AND RECOMMENDATIONS.............................................................61

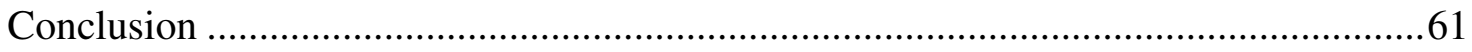

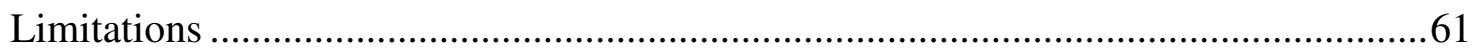


Recommendations for future study...

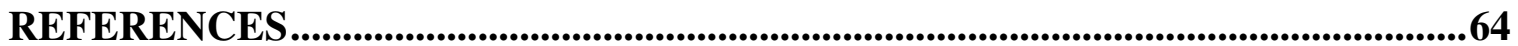

APPENDICES 


\section{LIST OF APPENDICES}

Appendix A Modified Ashworth Scale.....................................73

Appendix B Consent form................................................ 74

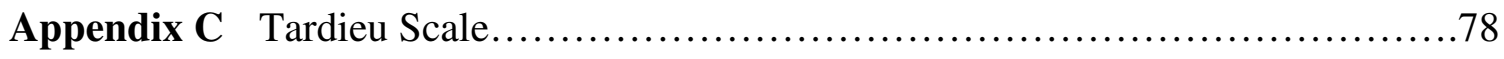

Appendix D BTX-A dosages for individual subjects........................... 79

Appendix E BTX-A dosage and injection guidelines.......................... 80

Appendix F Angular difference between baseline measures......................81

Appendix G Kinematic and kinetic profiles of individual subjects..................83 


\section{LIST OF FIGURES}

Figure 1 Components of the gait cycle.....................................

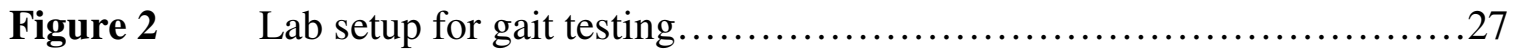

Figure 3 Standard configuration of infrared emitting diodes (IREDs)............29

Figure 4 Illustration of the instrumented probe............................. 31

Figure 5a Kinematics at the ankle, knee and hip of a "responder" at baseline, T1,

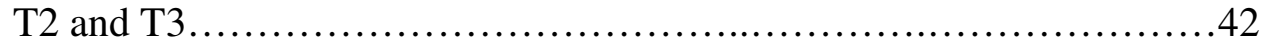

Figure 5b Kinematics at the ankle, knee and hip of a "non-responder" at baseline,

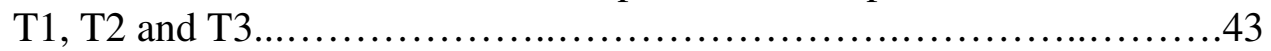

Figure 6a Powers $(\mathrm{W} / \mathrm{kg})$ at the ankle, knee and hip of a "responder" at baseline,

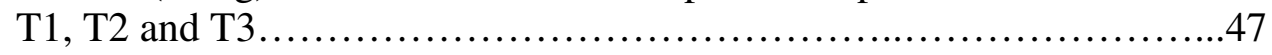

Figure 6b Powers (W/kg) at the ankle, knee and hip of a "non-responder" at baseline, T1, T2 and T3 


\section{LIST OF TABLES}

Table 1 Summary of subject demographics and stroke characteristics...........35

Table 2 Summary of clinical measures at baseline............................ 37

Table 3 Summary of clinical measures obtained on the affected side two weeks

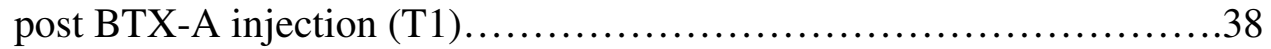

Table 4 Individual subject step length $(\mathrm{m})$, stance time $(\mathrm{sec})$ and gait speed $(\mathrm{m} / \mathrm{s})$

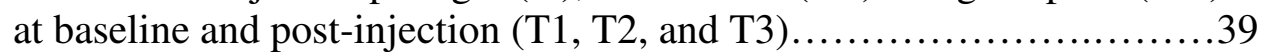

Table 5 Mean and standard deviations of temporal spatial parameters of gait at

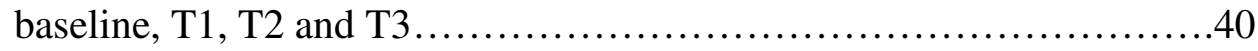

Table 6 Mean and standard deviations of kinematic measures of gait during stance at baseline and post-injection (T1, T2 and T3).

Table 7 Mean and standard deviations of power and work measures at the ankle and hip during stance at baseline and post-injection (T1, T2 and T3).....46

Table 8 Summary of individual clinical, kinematic and kinetic changes over time (T1 and T3) ..................................................49 


\section{GLOSSARY}

(Whittle, 1996; Winter, 1991)

Dorsiflexion: flexion of the foot in an upward direction.

Double support: the period of time during walking, between initial contact of one foot and toe off of the opposite foot, when both feet are in contact with ground.

Foot flat (FF): the instant during the stance phase when the foot is flat on the ground.

Gait: the manner of locomotion by changing location of the feet, with the condition that at least one foot is always in contact with the surface.

Gait cycle: the series of events which occur from initial contact of one foot to the subsequent contact of the same foot.

Gait velocity: the average horizontal speed of the body as it moves through the plane of progression. It is measured in metres/second $(\mathrm{m} / \mathrm{s})$.

Ground reaction force: equal in magnitude and opposite in direction to the force that the body exerts on the supporting surface through the support limb(s).

Heel off: the instant during the stance phase when the heel leaves the ground. The forefoot and toes are normally still in contact with the ground.

Initial contact (IC): the instant during the stance phase when the foot makes contact with the ground, independent of how that contact is made. 
Inverse dynamics link segment modeling: a method of analyzing human motion. In gait, the process begins with the foot in contact with the ground and progressively moves up the chain. Limbs are considered rigid segments and joints frictionless links. Using kinematic data and ground reaction forces, Newtonian laws of motion are applied to compute the net moments, powers and work generated by the muscles crossing a joint. Inverse dynamics analysis does not provide any indication of the contribution of individual muscles, but rather determines the net effect.

Kinematics: the branch of mechanics that refers to the spatial movement of the body, limbs, and joints independent of the forces that cause the movement.

Kinetics: the branch of mechanics that refers to the forces that cause the movement

Mechanical power: is the work performed per unit of time and provides information about the energy generated and absorbed by the muscles about a joint during concentric or eccentric contractions. It is measured in Watts (W).

Midstance: the instant during the stance phase when the body is directly over the supporting limb.

Moment of force: refers to the product of a force acting at a distance about an axis of rotation, causing angular acceleration about that axis. It is measured in Newton-meters (N.m.). 
Negative work: equals the time integral of the joint mechanical power when it is negative (when the muscle is lengthening) and is the work done on an eccentrically contracting muscle. It is measured in Joules ( $\mathrm{J})$.

Plantarflexion: movement that flexes the foot in the downward direction.

Positive work: equals the time integral of the joint mechanical power when it is positive (when the muscle is shortening) and is the work done by a concentrically contracting muscle. It is measured in Joules ( $\mathrm{J}$ ).

Push off (PO): the period of time in late stance when plantarflexion normally occurs and the lower limb is pushing away from the ground.

Single support: the period of time during walking, when only one limb is in contact with the ground, and is exactly equal to the swing phase of the contralateral limb.

Stance phase: the period of time during the gait cycle from initial contact to toe off of the same foot, when the foot is in contact with the ground.

Swing phase: the period of time during the gait cycle, occurring from toe off to initial contact of the same foot, when the foot is not in contact with the ground.

Toe off (TO): the instant during the stance phase when the toe of the foot leaves the ground. Toe off identifies the end of the stance phase and beginning of the swing phase. 


\section{ABBREVIATIONS}

MaxDF: Maximum dorsiflexion angle (degrees)

MaxPF: Maximum plantarflexion angle (degrees)

MaxFlex: Maximum flexion angle (degrees)

MaxExt: Maximum extension angle (degrees)

A: Affected side

UA: Unaffected side

A:UA: Ratio of affected side to unaffected side

UA-A: Difference between the unaffected and affected sides

AROM: Active range of motion

PROM: Passive range of motion

MAS: Modified Ashworth Scale

B1: First baseline test session (baseline 1)

B2: Second baseline test session (baseline 2)

B: Average baseline

T1: Two weeks post-injection test session

T2: Six weeks post-injection test session

T3: Ten weeks post-injection test session

Ankle Max Gen: Maximum ankle power generation (W/kg)

Ankle Max Abs: Maximum ankle power absorption (W/kg)

Total +ve work: Total positive work (total power generation; J/kg)

Total -ve work: Total negative work (total power absorption; $\mathrm{J} / \mathrm{kg}$ ) 


\section{CHAPTER 1}

\section{INTRODUCTION}

Stroke, also known as a cerebrovascular accident, is a sudden loss of brain function caused by an interruption of blood flow to the brain (ischemic stroke) or rupture of the blood vessels in the brain (hemorrhagic stroke) (Nolte, 2002). Stroke is the fourth leading cause of death among Canadians. It is also the leading cause of adult neurological disability and newly affects approximately 50,000 Canadians annually (Heart and Stroke Facts, 2003). Depending on the location and extent of damage to the brain tissue, the effects of stroke are variable and can range from sensory, motor, mental, perceptual and language impairments (O'Sullivan, 1994).

Motor impairment typically involves paresis of the side of the body opposite to the site of the brain lesion. Gait is a major determinant of independent living, therefore, it is not surprising that improvement of walking function is the most commonly stated priority of stroke survivors (Bohannon et al., 1988; Bohannon et al., 1991). Approximately $80 \%$ of stroke survivors achieve this goal (Wade et al., 1987) though the quality of walking performance often limits endurance and quality of life (Niemi et al., 1988; Mayo et al., 1999). Considerable time and resources by both patients and clinicians are invested into rehabilitation focused on the restoration of walking ability following stroke and reducing functional dependence.

Weakness of the plantarflexors is common following stroke (Nadeau et al., 1999; Parvateneni et al., 2007) impairing force generation. As well, hypertonicity of the ankle plantarflexors has been shown to occur in approximately $40 \%$ of those with chronic 
stroke (Watkins et al., 2002), which, if extreme, can restrict joint mobility and further contribute to the reduced capacity to generate force. From a biomechanics perspective, plantarflexor stiffness can limit the ability of the body to rotate over the stance limb due to reductions in dorsiflexion mobility attributable to passive resistance of the plantarflexor muscles. As well, the ability to push-off at the end of stance is affected, resulting in compensatory hip flexion to pull the foot off the ground (Olney et al., 1996). The elevated energy requirements needed to maintain this abnormal gait pattern can be a limiting factor in carrying out activities of daily living, underscoring the importance of reducing impairments causal to mobility limitation.

Botulinum Toxin A (BTX-A) has recently become a common clinical treatment used to reduce hypertonicity following stroke. BTX-A is administered by intramuscular injection to produce dose-related muscle weakness by binding to the cholinergic motor neuron terminals blocking the release of acetylcholine from the presynaptic vesicles at the neuromuscular junction (Blasi et al., 1993; Burgen et al., 1949; Gunderson, 1980). BTXA is shown to be a safe and cost effective method of treating many common forms of muscle overactivity (Esquenazi, 2006; Naumann et al., 2006; Turkel et al., 2006). However, studies evaluating the effectiveness of BTX-A treatment in stroke are limited. Although improvements following BTX-A have been shown, the studies have been quite superficial in terms of the limited outcomes measured. BTX-A has been shown to result in tone reduction as indicated by the Ashworth or modified Ashworth scale (Bergfeldt et al., 2006; Francisco et al., 2002; Mancini et al., 2005), gains in active and passive ankle range of motion (Bayram et al., 2006; Dengler et al., 1992; Pierson et al., 1996), and subjectively, patients report improvement (Bergfeldt et al., 2006). However, the impact of 
these clinical changes on function remains largely unknown and the biomechanical effectiveness of BTX-A treatment in the ankle plantarflexors in chronic stroke has not been well addressed.

Some studies have reported improvements in gait speed and gains in stride length following BTX-A injection of the ankle plantarflexors (Fock et al., 2004; Hesse et al., 1994; Johnson et al., 2004), but the findings are not necessarily repeatable in similar populations (Burbaud et al., 1996; Cioni et al., 2006; Dengler et al., 1992). This may be because the tone reduction was not of a sufficient magnitude to manifest a change in such a general measure of function as gait speed, or that tone was itself not a limiting factor to mobility. In order to appreciate and fully understand the effects of BTX-A treatment on walking performance, detailed biomechanical gait analysis is necessary to quantify the motion patterns of each lower limb joint and the net effect of muscles acting about those joints to produce the overall pattern of walking. In this manner, changes induced at the ankle may be detected and not masked by the insensitivity or lack of resolution of general clinical measures.

The purpose of this study was to describe the gait patterns in subjects prior to and after BTX-A treatment of the ankle plantarflexors. By describing the kinematic and kinetic patterns of walking, the influence of BTX-A on gait efficiency can be determined and the clinical factors that predispose stroke patients to a positive treatment outcome can be more fully understood. 


\section{CHAPTER 2}

\section{LITERATURE REVIEW}

Gait is one of the most common and complex of human movements (Winter, 1991). The ability to walk is generally associated with the achievement of four related tasks: maintenance of upright posture; balance or stance stability during the stance phase of the gait cycle; clearance of the swinging foot during the swing phase of the gait cycle; and the supply of sufficient energy to the body with each stride to ensure efficient forward progression (Olney and Richards, 1996; Winter, 1991). Safe and efficient walking requires appropriate neuromuscular control in order to accomplish the tasks (Winter, 1991). In individuals with impaired motor systems, such as those with stroke, deficiencies in performing these tasks may present significant challenges to mobility, resulting in abnormal and inefficient gait patterns. Gait analysis can provide valuable information for understanding the biomechanics and sourcing the problems causing pathological gait and evaluating the effectiveness of different treatment interventions.

\section{The gait cycle}

The gait cycle is a series of events which occur from initial contact of one foot to the subsequent initial contact of the same foot. It is divided into the stance and swing phases, which represent approximately $60 \%$ and $40 \%$ of the gait cycle, respectively. Winter (1991) describes the phases of the gait cycle in the following manner: the stance phase is the period of time when the foot is in contact with the ground, and is subdivided into initial contact, foot flat, midstance, heel off and toe off. The swing phase begins with 
toe off and is the period during which the foot is not in contact with the ground. During the gait cycle there are two periods of double support where both feet are in contact with the ground, and two periods of single support where only one limb is in contact with the ground such that the body's entire weight rests upon one extremity. Figure 1 illustrates the gait cycle and the relative timing of its components.
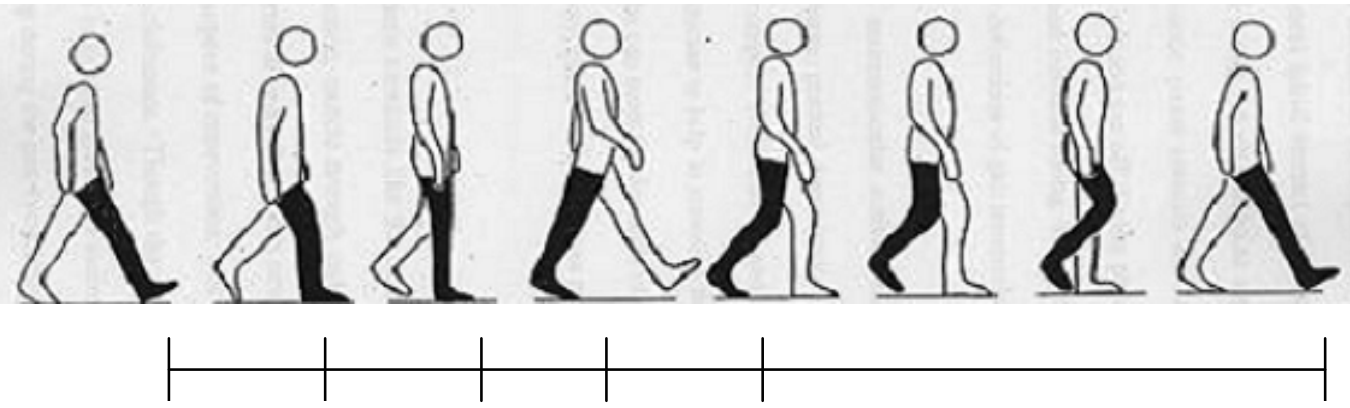

$0 \%$
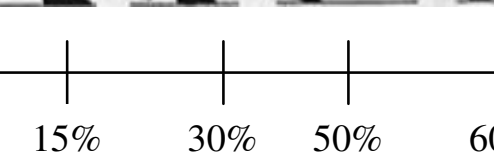
contact

$30 \% \quad 50 \% \quad 60 \%$

$100 \%$

Initial

stance

contact
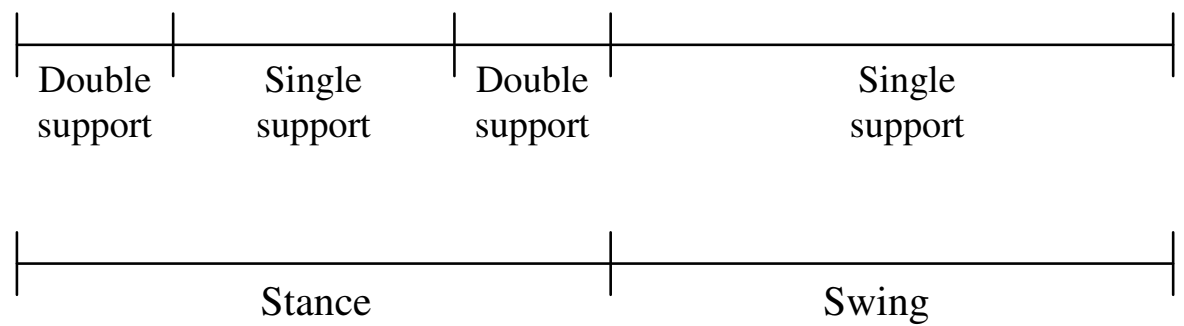

Figure 1 Components of the gait cycle (Modified from Winter, 1987)

\section{Kinematics of gait}

Kinematics refers to the spatial movement of a body or systems of bodies independent of the forces that cause the movement (Winter, 1991). Motions commonly described include linear and angular joint positions, velocities and accelerations. Throughout the gait cycle, the kinematics of a joint provide information about the 
position and direction of movement. The ankle, knee and hip joint angles in the sagittal plane for normal, healthy individuals have been well described as they, in combination, result in forward progression. The trajectories are summarized in the following section.

\section{Normal kinematics}

At initial contact the ankle is typically within a few degrees of the neutral position $\left(90^{\circ}\right.$ angle between the foot and shank). As the foot is lowered to the ground, and the body's weight is transferred onto the limb in early stance (foot flat), the ankle joint achieves about $5^{\circ}$ of plantarflexion and then returns to neutral. The ankle reaches approximately $10^{\circ}$ of dorsiflexion as the tibia rotates forward over the foot. During late stance, the body's weight is transferred to the opposite limb and the ankle plantarflexes to approximately $20^{\circ}$ in order to move the body and limb forward by pushing the leg off of the ground and beginning the swing phase (Winter, 1991).

The knee joint is typically in about $5^{\circ}$ of flexion at initial contact, but rapidly flexes during early stance to approximately $20^{\circ}$. During midstance, as the body rotates over the limb the knee joint angle decreases (extends) from about $20^{\circ}$ of flexion to full extension. The knee then flexes during late stance, reaching a peak of about $65^{\circ}$ during initial swing in order to assist the foot with ground clearance (Winter, 1991).

The hip is normally flexed about $20^{\circ}$ at initial contact. The hip begins to extend during early stance, reaches neutral at midstance, and continues extending until it reaches a peak extension of approximately $12^{\circ}$ of extension just before the end of the stance phase. Hip flexion then occurs to assist with bringing the limb forward through the swing phase of gait (Winter, 1991). 
The patterns of joint excursion are highly reproducible from stride to stride (Winter, 1991) and are consistent across healthy adults walking at a natural, self-selected speed (Winter, 1983) with an average variability over the stride reported to be less than $2^{\circ}$ at all joints (Winter, 1991).

\section{Kinetics of gait}

Kinetics refers to the study of the forces and moments that cause movement. In order to calculate joint kinetics, the ground reaction forces, including both magnitude and direction, are recorded during the gait cycle. The combination of these external forces, anthropometric data (mass, geometry, moment of inertia and centre of gravity for each segment), and the known dynamics of each body or limb segment (kinematics), allows for the calculation of the instantaneous moments of force, joint mechanical powers and work about each joint throughout the gait cycle. Inverse dynamics link segment modeling, ie. calculating these parameters from distal to proximal and treating limbs as rigid segments connected by joints or links, provides the framework to which Newton's laws of motion are applied. The derived information assists in understanding the underlying cause of the movement.

The moment of force (measured in Newton-meters, $\mathrm{Nm}$ ) is the product of a force acting at a distance about an axis of rotation, causing a rotational effect about that axis (Winter, 1991). The moments of force commonly calculated reflect the net effect of all agonist and antagonist muscles acting across a joint and supply information about which muscle group is providing the dominant moment (flexors or extensors). It is important to note that there are many combinations of muscles generating force that can result in 
similar movement goals. This variability provides for the flexibility and adaptability of the neuromuscular system and is very purposeful in pathological gait to compensate for motor deficits (Winter, 1984).

The product of the net muscle moment and the angular velocity quantifies the muscle power (Winter, 1991).

$$
\mathrm{P}_{\mathrm{j}}=\mathrm{M}_{\mathrm{j}} \cdot \omega_{\mathrm{j}}
$$

where $P_{j}$ is the power in watts, $\mathbf{M}_{j}$ is the net joint moment in Newton meters and $\omega_{j}$ is the joint angular velocity in radians per second. Mechanical power provides information about the energy generated and absorbed by the muscles about a joint during concentric or eccentric contractions in order to accomplish work to produce movement. Positive power, or power generation, occurs if the moment acts in the same direction as the angular velocity. Negative power, or power absorption is the opposite; the moment acts in the opposite direction as the angular velocity (eccentric).

Power is the rate of doing work. Therefore, the integral of the power curve over time provides a measure of the work (Joules) performed by a muscle group crossing a particular joint. Positive work is the work done by concentrically contracting muscles. The net positive work done by the muscles crossing a joint is the time integral of the joint mechanical power when it is positive, and represents generated energy transferred from the muscles to the limbs. Similarly, the net negative work is the work done by eccentrically contracting muscles and is equal to the time integral of the joint mechanical power when it is negative. Negative work represents a flow of energy from the limb motion into the muscles (Winter, 2005). The kinetics of movement cause the kinematic and temporal spatial patterns of walking and therefore provide valuable information about 
the control of gait. Examination of the power profiles of walking provides insight of net rate of generating or absorbing energy by all muscles and other connective tissue crossing the joint (Winter, 1991).

\section{Normal kinetics}

The internal moments of force and joint power profiles of healthy adults in the sagittal plane were well described by Winter (1991). He also introduced the concept of support moment, which is the algebraic sum of the instantaneous lower limb joint moments during stance. The support moment must be extensor to maintain an upright posture otherwise the individual would be collapsing. Implicit is that it is important to consider the actions at all lower limb joints to appreciate their collective impact on walking stability.

With the exception of early stance, a plantarflexor (extensor) moment is evident throughout the stance phase and contributes to a major power burst at push off. At the knee the flexors predominate in early stance leaving the hip to provide the supportive extensor moment and power generation. During midstance hip flexors are active while the hip is extending to provide power absorption while the knee extensors contribute to power absorption as the knee flexes. At pushoff, the hip contributes to the power generated as the flexors concentrically contract, termed pull off.

The stereotypical nature of the kinematic and kinetic profiles of young and old adults (Winter, 1991) enables abnormalities to be readily identified. Indeed gait analysis has been instrumental in localizing deficits to a particular joint or muscle group and has 
quantified the impact of training regimens on gait performance in people with disabilities such as stroke (Parvataneni et al., 2007).

\section{Stroke}

A stroke, also known as a cerebrovascular accident (CVA), is a sudden loss of brain function caused by an interruption of blood flow to the brain (ischemic stroke) or rupture of the blood vessels in the brain (hemorrhagic stroke) (Nolte, 2002). Following stroke, a variety of deficits may occur, including sensory, motor, mental, perceptual and language impairments (O'Sullivan, 1994). Stroke, which is the fourth leading cause of death among Canadians, is the leading cause of adult neurological disability and affects approximately 50,000 Canadians annually (Heart and Stroke Facts, 2003).

Regaining the ability to walk is the most common stated priority following stroke (Bohannon et al., 1988; Bohannon et al., 1991) and about $80 \%$ of survivors achieve this goal (Wade et al., 1987). Many, however, require the use of walking aids and have limited efficiency and endurance which impact quality of life and social participation (Jorgensen et al., 1995; Mayo et al, 1999).

From a biomechanics perspective, the ability of stroke survivors to regain normal walking depends on strength, joint mobility, coordination and aerobic capacity (Nadeau et al., 1999; Knuttsson and Richards, 1979; Macko et al., 1997). The challenge is to determine which impairments should be targeted in order to enhance walking performance. To address this, a better understanding of the gait patterns of people with stroke is needed. 


\section{Stroke and gait}

Paresis frequently results from hemispheric stroke and reduces the patient's walking ability (Chen et al., 2005; Goldie et al., 2001). The most commonly stated priority of the individuals following acute stroke is the return of their ability to walk (Bohannon et al., 1988; Bohannon et al., 1991). Reduced mobility contributes to loss of independence (Perry et al., 1995) and reduced quality of life (Suzuki et al., 2002) and therefore the need to characterize and retrain gait following stroke is a major goal of rehabilitation programs.

\section{Temporal spatial variables and stroke}

Many studies have quantified temporal-spatial gait parameters following stroke, including gait velocity, step length, stride length, symmetry ratios, and gait phase durations (Goldie et al., 2001; Kim and Eng, 2004; Lin et al., 2006; Olney et al., 1994). It has been suggested that these variables are useful to clinically assess gait performance and monitor functional recovery or decline following stroke (Andriacchi et al., 1977; Mizrahi et al., 1982; Roth et al., 1997).

Individuals with stroke walk significantly more slowly than their able-bodied counterparts with average speeds ranging from $0.16 \mathrm{~m} / \mathrm{s}$ to $0.86 \mathrm{~m} / \mathrm{s}$ (Chen et al., 2005; Hsu et al., 2003; Huitema et al., 2004; Kim and Eng, 2004; Lehman et al., 1987; Lin et al., 2006; Nadeau et al., 1999; Olney et al., 1986; Olney et al., 1991; Teixeira-Salmela et al., 2001). The range in gait velocity reflects differences in stroke severity with greater severity associated with slower speeds; though even those mildly affected walk slower 
than healthy adults of similar ages $(1.27 \mathrm{~m} / \mathrm{s}$ to $1.39 \mathrm{~m} / \mathrm{s}$ for men and women aged 50 to 70; Bohannon, 1997).

In addition to decreased walking speed, both cadence and step length are lower than observed in able-bodied individuals. Excessive plantarflexor activation elicited by stretching the spastic gastrocnemius during weight transfer to the affected leg causes difficulty in moving the body's centre of gravity forward for the next step, correlating with a shorter step length of the less affected (hereafter referred to as 'unaffected') limb (Lin et al., 2006). Further, the stance phase on the affected side is shorter than on the unaffected side, but both are longer compared to able-bodied individuals and account for an abnormally high proportion of the full gait cycle (stance to swing ratio: 67:33 and 80:20 for the affected and unaffected sides, respectively; normal ratio: 62:38; Peat et al., 1976). The prolonged stance phase has been attributed to difficulty in advancing the leg during swing phase due to diminished strength and decreased power generated at push-off at the ankle (Goldie et al., 2001; Olney and Richards, 1996). The longer period of double support is further indication of the difficulty in transferring body weight to the affected limb due to reduced push-off power, impaired balance, or both (Goldie et al., 2001).

Gait speed is a sensitive and reliable measure in persons with stroke (Richards et al., 1995; Roth et al., 1997). It is an important descriptor of gait performance and increased gait speed relates to improved functional ability and independence (Potter et al., 1995; Teixerira-Salmela et al., 2000), however characterization of the degree of asymmetry as noted by side-to-side differences in both temporal and spatial measures provide insight into the efficiency of walking (Lin et al., 2006). Asymmetry is a hallmark 
of hemiplegic gait (Olney and Richards, 1996) and is independent of gait velocity (Wall and Turnball, 1986; Roth et al., 1997).

\section{Kinematic variables and stroke}

Although hemiplegic gait may vary greatly from individual to individual, there are distinct movement patterns which have been observed and associated with specific physical impairments. In general, there is a decrease in total hip, knee and ankle joint excursions during stance compared to healthy individuals (Knutsson and Richards, 1979; Lehman et al., 1987; Olney et al., 1991). On the affected side, subjects with stroke characteristically show less hip flexion, more knee flexion and less ankle plantarflexion at initial contact compared to healthy individuals though knee extension has also been reported at initial contact (Olney and Richards, 1996; Kerrigan et al., 1999). Knee hyperextension, less hip flexion and more ankle plantarflexion than normal is typical throughout midstance.

These commonly described deviations at the ankle and knee from healthy gait have been associated with increased stiffness and spasticity of the ankle plantarflexors and agonist-antagonist coactivation at the ankle (Knutsson and Richards, 1979; Lamontagne et al., 2002). Abnormalities at the hip may compensate deficits at the ankle (Nadeau et al., 1999; Olney et al., 1996). The smaller than normal angular displacements may also reflect an adaptation to impaired balance or instability as the individual attempts to control their forward progression by reducing mobility (Olney and Richards, 1996). 


\section{Kinetic variables and stroke}

Knowledge of the kinetics of the gait of stroke patients offers insight into the nature of gait deficiencies, beyond what kinematics alone may offer, and helps to provide a richer understanding of the effects of different rehabilitation strategies (Olney et al., 1988; Olney and Richards, 1996). In terms of joint moments, the affected side of 'fast' walkers (mean gait speed: $0.63 \mathrm{~m} / \mathrm{s}$ ) has been shown to generate large hip flexor moments in late stance, a knee moment biased towards extension and either small dorsiflexor or plantarflexor moments at the ankle (Olney et al., 1996). The knee extensor moment contributes to the overall support of the body whereas the hip flexors facilitate limb pulloff, in part to compensate for limited plantarflexor push-off (Nadeau et al., 1999; Olney and Richards, 1996). Both the hip and the knee moment profiles for slow walkers (mean gait speed $0.25 \mathrm{~m} / \mathrm{s}$ ) show exaggerated extensor moments throughout most of stance on the affected side with little contribution from the ankle musculature (Olney et al., 1994).

The joint power profiles generated throughout walking in stroke are quite similar in shape to healthy individuals, but the amplitude on both the affected and unaffected sides are lower and asymmetrical suggesting that the unaffected side may be compensating to some degree (Olney et al., 1996; Parvataneni et al., 2007). In fact, the unaffected side performs about $60 \%$ of the positive work (Olney et al., 1991) which is likely to offset weakness on the affected side (Parvataneni et al., 2007). The ankle joint normally contributes about 80 to $85 \%$ of the total power generated during the entire gait cycle (Winter, 1991). In stroke, the same is true in fast walkers as the pushoff power was the largest contributor to the positive work accomplished on both sides (Olney et al., 
1991; Kim and Eng, 2004; Parvataneni et al., 2007), although in slower walkers the power burst was shown to be barely evident on the affected side (Olney et al., 1991).

The abnormally large burst of ankle power on the unaffected side compared to the affected side may be an adaptation to provide additional power generation for walking to compensate for the low power generation on the affected side (Olney and Richards, 1996) and maximize gait speed (Kim and Eng, 2004). Compensation at the hip occurs in response to the diminished strength of the ankle plantarflexors. Higher than normal hip flexor activity at pull off (late stance) provides additional power to the system to perform the work of walking and compensates for the low contribution of the ankle musculature (Nadeau et al., 1999; Olney and Richards, 1996).

It is well established that the ankle plantarflexors are a major contributor to the work of walking in healthy gait (Winter, 1991) and following stroke (Olney et al., 1991; Kim and Eng, 2004; Parvataneni et al., 2007). The power generated on the affected side by these muscles and the work performed is directly related to the speed of walking (Kim and Eng, 2004; Parvataneni et al., 2007). It follows that interventions aimed at improving plantarflexor function can result in marked improvements in gait.

The plantarflexors are generally very weak following stroke (Kim and Eng, 2003; Nadeau et al., 1999) and coactivation with dorsiflexors further limits torque production (Levin and Hui-Chan, 1994). Additionally, the plantarflexors are hypertonic in about $40 \%$ of those with chronic stroke (Watkins et al., 2002), which, if extreme, can resist joint mobility and further contribute to the reduced capacity to generate force. The factors that contribute to elevated muscle tone include hyper-reflexia (or spasticity), alterations in passive structures that increase stiffness, and alterations to the intrinsic properties of the 
muscle (Dietz et al., 1991; Katz and Rymer, 1989; Rydahl and Brouwer, 2004; Sinkjaer and Magnussen, 1994; Thilmann et al., 1991). Several investigators have demonstrated that non-reflex mediated stiffness, rather than stretch reflex enhancement, is largely responsible for hypertonia (Dietz et al., 1991; Lee et al., 1987; Rydahl and Brouwer, 2004; Sinkjaer and Magnussen 1994). However, despite identification of a primary mechanism of hypertonia, several factors can coexist; they are interdependent and change over time. Some stroke survivors report mobility reducing spasms and painful contractures (O'Dwyer et al., 1996). Immobilization due to hypertonia can lead to further changes in muscle connective tissue as collagen accumulates within the muscle resulting further in increased passive stiffness (Given et al., 1995; Booth et al., 2001).

Chronic hypertonia of the ankle plantarflexors generally is associated with a reduced capacity to generate force, not due to the inability to recruit motor units (a primary deficit following stroke) but altered muscle mechanics. The shortened plantarflexors are less able to form cross bridges thus reducing tension development (Williams and Goldspink, 1978). In addition, the corresponding lengthened dorsiflexors also generate less tension than is possible at their optimal length (Brouwer et al., 1998). The resultant weakness of the ankle musculature impacts negatively on balance, stability and gait. If tone could be normalized this could improve joint mobility and function. Botulinum Toxin A (BTX-A) is a pharmacological agent that reduces reflex mediated and intrinsic muscle stiffness. 


\section{Botulinum Toxin A (BTX-A)}

\section{Physiological effects}

BTX-A is one of seven immunologically distinct forms of botulinum neurotoxin produced by the bacterium, Clostridium botulinum (Pearce et al., 1997). BTX-A is administered by intramuscular injection to produce dose-related muscle weakness by binding with high affinity and specificity to the cholinergic motor neuron terminals blocking the calcium mediated release of acetylcholine from the presynaptic vesicles at the neuromuscular junction (Blasi et al., 1993; Burgen et al., 1949; Gunderson, 1980). In addition to exerting an effect on the alpha-motor neuron innervated extrafusal muscle fibers, evidence indicates that BTX-A affects the gamma-motor neuron innervated intrafusal muscle spindles as well (Rosales et al., 1996; On et al., 1999). This is important since elevated gamma-motor neuron activity has been implicated in spasticity as it increases the excitability of the spindle afferents which in turn increase their sensitivity to stretch (Katz and Rymer, 1989). Reducing the activation of gamma-motor neurons will result in a decrease in spindle excitability to stretch thus resulting in less firing of alphamotor neurons. The reduced reflex activity paired with a decrease in alpha-motor neuron activation can reduce intrinsic stiffness.

Following BTX-A injection, there is a 24 to 72 hour delay before the onset of observable clinical effects, which have been shown to last from 2 to 6 months (Davis and Johnson, 2001), peaking after about 2 weeks to 8 weeks post injection (Corry et al., 1998; Francisco et al., 2002; Hesse et al., 1994; Mancini et al., 2005; Rousseaux et al., 2005; Suputtitada and Suwanwela, 2005). 


\section{BTX-A and gait}

Many studies have quantitatively investigated the clinical and biomechanical effects of BTX-A in children with cerebral palsy (CP). Clinical assessment using the Ashworth scale has shown significant reduction in tone from one month to 4 months post BTX injection (Bottos et al., 2003; Sarioglu et al., 2003) Improvements in observed gait (Koman et al., 2000, Sarioglu et al., 2003), and significant gains in the amount of ankle dorsiflexion at initial contact, and peak ankle dorsiflexion angle achieved in stance and swing have been documented following BTX-A treatment (Boyd et al., 2000; Cosgrove and Graham, 1998; Galli et al., 2001; Papadonikolakis et al., 2003; Sutherland et al., 1998; Zurcher et al., 2001).

Sutherland et al. (1998) quantified kinematic changes in walking performance following BTX-A injection in the gastrocnemius muscle using three dimensional gait analysis in a double-blind control study of 20 ambulatory children with cerebral palsy. They reported significantly increased dorsiflexion at $10 \%$ of the gait cycle (mean increase of $\left.5.4^{\circ}\right)$, significantly increased peak dorsiflexion in stance $\left(12.5^{\circ}\right)$, and peak dorsiflexion in swing $\left(6.2^{\circ}\right)$ in the group that received BTX-A injections 8 weeks prior. They

attributed these improvements to increased compliance of the ankle plantar flexor muscles; no improvements were detected in the control group. Others have shown that at about eight weeks post BTX-A injection of the gastrocnemius muscle, knee extension at initial contact normalized, demonstrating less hyperextension (average decrease of $8.3^{\circ}$ ) (Zurcher et al., 2001).

Only a few studies have reported kinetic changes following BTX-A treatment. Normalization of sagittal plane moment and power patterns at the ankle were 
documented, from 3 to 24 weeks post plantarflexor injection (Boyd et al., 2000; Galli et al., 2001; Zurcher et al., 2001). These changes were attributed to a more neutral position of the ankle in initial stance, resulting in a tendency for a delay in activation of the stretch reflex which tended to occur more appropriately at midstance and contribute to increased forward propulsion (Boyd et al., 2000). In contrast, Bottos et al. (2003) investigated the effects of BTX-A in two groups: one group receiving BTX-A only and another receiving BTX-A followed by casting. Gait analysis was performed pre-treatment and 1, 4 and 12 months post treatment. Although a reduction in tone (as determined by the Ashworth scale) was found in both groups post-treatment, the authors found no kinematic or kinetic changes following treatment. The authors attributed the largely negative findings to a limited number of subjects in both groups and a non ideal selection of children to undergo for gait analysis; height and weight parameters may not have been ideal for 3D gait analysis and differed from those recommended by Boyd, 2000. As well, Bottos et al. (2003) included only children with mild spastic diplegia, whereas Boyd et al. (2000) had included children with spastic hemiplegic or diplegic cerebral palsy who exhibited signs of dynamic equinus affecting gait. It seems that the children in Boyd et al.'s (2000) study were more severely hypertonic and therefore may have been more responsive to the intervention.

The effects of BTX-A on gait in stroke have also been investigated, with findings similar to those in children with cerebral palsy. BTX-A has been shown to be an effective treatment for reducing plantarflexor muscle spasticity following stroke and improving function (Bayram et al., 2006; Bergfeldt et al., 2006; Burbaud et al., 1996; Cioni et al., 2006; Dengler et al., 1992; Dunne et al., 1995; Hesse et al., 1996; Mancini et 
al., 2005; Rousseaux et al., 2005; Pierson et al., 1996). Several studies have shown that BTX-A injections given within one year of the stroke produce clinical improvements such as a reduction in tone as indicated by the Ashworth scale and active gains in ankle dorsiflexion (Burbaud et al., 1996; Rousseaux et al., 2005). Others have also shown improved function in subjects with longer term spasticity or hypertonicity (Dunne et al., 1995; Hesse et al., 1996). It has been suggested that subjects who walk faster benefit more from BTX-A treatment following the study by Cioni et al. (2006). They investigated the temporal spatial gait parameters before and 4 weeks after BTX-A injection in a slow walking group ( 0.18 to $0.49 \mathrm{~m} / \mathrm{s})$ and a medium speed walking group $(0.50$ to $0.99 \mathrm{~m} / \mathrm{s})$. Because the group with higher mobility function (medium walking velocity) exhibited a reduction in the base of support during walking (step width) whereas the other group showed no change they concluded that baseline functional ability was a determinant of BTX-A effectiveness (Cioni et al., 2006). Unfortunately they did not measure tone or joint mobility pre and post intervention as a more direct indication of the drug's action.

Studies evaluating the effectiveness of BTX-A treatment in stroke are limited. Although improvements following BTX-A have been shown, the studies have been quite superficial in terms of the outcomes measured. As expected BTX-A results in tone reduction, as indicated by the Ashworth or modified Ashworth scale (Bergfeldt et al., 2006; Francisco et al., 2002; Mancini et al., 2005), gains in active and passive ankle range of motion (Bayram et al., 2006; Dengler et al., 1992; Pierson et al., 1996), and subjective patient reports of improvement (Bergfeldt et al., 2006). However, the impact of reducing impairments on function remains largely unknown. Detailed investigation incorporating 
measures of specific joint function as well as mobility may provide greater insight into the effects of BTX-A treatment.

Few studies have directly explored the effects of BTX-A treatment on muscle function. Hesse et al. (1996) measured tone and surface electromyography (EMG) of the plantarflexor muscles during gait before BTX-A treatment and four weeks after the injection. In this study, 9 out of 12 subjects showed a reduction in tone of at least one point on the modified Ashworth scale. These same individuals showed increases in gait velocity, stride length and cadence averaging $33.3 \%, 16.9 \%$ and $15.7 \%$, respectively. Further, there was an average of $35.3 \%$ less premature activity of the soleus muscle (defined as the activity of the soleus muscle during terminal swing and initial contact: $90 \%$ to $110 \%$ of the normalized gait cycle) and a reduction muscle activity during midstance by an average of $38.6 \%$. The three non-responders showed no change in Ashworth score, gait velocity, or soleus muscle activity and two of them showed a completely disrupted muscle activation pattern involving several limb muscles as described by Knuttson and Richards, 1979. Perhaps when deficits are widespread, the benefits of interventions targeting a specific joint are masked.

An earlier study by Hesse et al. (1994) examined the trajectories of the vertical ground reaction force vector under the feet. They found that 2 weeks following BTX-A injection the force vector shifted from initial forefoot contact (40\% of the foot length measured from the toe, which is defined as $0 \%)$ to flat foot with the affected leg $(60 \%$ of the foot length), supporting improved body advancement by lengthening stance. Further, qualitatively the vertical force profiles appeared more normal, with higher push off forces on the affected side 2 weeks after the injection. The authors speculated that lower muscle 
tone (lower Ashworth score) might be due to an elevated threshold of the stretch reflex, which may have improved the progression of the body over the support limb and increasing the stride length. The effects diminished after 8 weeks. It is not clear if the changes in vertical ground reaction force profiles corresponded to altered ankle joint kinematics. Like most studies to date measuring joint angles or reaction forces independently restricts the ability of the authors to draw conclusions. To better understand the effectiveness of BTX-A treatment of the ankle plantarflexors a comprehensive approach that links kinematic outcomes to the ability of the muscles to produce the work of gait efficiently is needed.

\section{Purpose of the study}

The purpose of the study was to describe and compare the gait parameters in persons with hypertonia secondary to stroke prior to and after BTX-A injection of the ankle plantarflexors. Specifically, the study describes walking kinematics and kinetics to determine the impact of BTX-A on ankle function in particular and on the gait pattern more generally. As well, the study sought to identify clinical characteristics associated with subjects who responded positively to the treatment in terms of gait performance and those who did not. 


\section{CHAPTER 3}

\section{METHODOLOGY}

\section{Study design}

This study was a single group, open-label trial. A repeated measures design including multiple baseline and three post-intervention time points was adopted to determine the effect of BTX-A in the ankle plantarflexors on gait parameters over time.

\section{Subjects}

For this pilot project, a convenience sample of seven subjects was recruited over an 18 month period by the attending physician (Dr. Stephen Bagg) at the outpatient Stroke Clinic at St. Mary's of the Lake Hospital, Kingston, Ontario.

\section{Inclusion criteria}

All subjects who met the following inclusion criteria were considered for the study:

1) A minimum of 6 months post hemispheric stroke;

2) Ability to walk independently a distance of 10 metres (ankle foot orthoses/canes permitted for entrance into the study although not permitted throughout testing);

3) Have moderate to severe plantarflexor hypertonicity in the affected limb, as determined by a score of 2 or greater on the Modified Ashworth Scale (Appendix A);

4) Stable physical health;

5) Signed consent form (Appendix B). 


\section{Exclusion criteria}

Subjects were excluded if they had any of the following characteristics:

1) A prior stroke affecting the contralateral hemisphere, brainstem or ipsilateral cerebellum;

2) A neurological condition other than stroke;

3) A lower extremity orthopaedic condition, or other medical condition affecting walking ability;

4) Notable cognitive deficits, as determined by physician assessment, that would hinder the ability to understand or follow instructions;

5) Known sensitivity to the study medication;

6) Received a BTX-A injection within four months prior to the clinic visit;

7) Unable to commit to the twelve week study period.

\section{Clinical measures}

The clinical measures of muscle stiffness, active range of motion and passive range of motion were selected based on those currently used in the literature to assess functional improvements post BTX-A injection (Burbaud et al., 1996; Hesse et al., 1994; Pierson et al., 1996; Reiter et al., 1998).

\section{Muscle stiffness measures}

Muscle stiffness of the affected ankle was measured using the Modified Ashworth Scale (Appendix A) and Tardieu scale (Appendix C) before and after BTX-A treatment. The Tardieu scale consists of two parts. The first part of the scale describes the muscle's 
reaction to stretch, by grading the response on a scale of 0 to $4(0=$ no resistance, $1=$ slight resistance, no catch, $2=$ catch and release, $3=$ fatigable clonus, $4=$ infatigable clonus). The second part of the scale measures the angle at which the muscle reacts to stretch. The relative difference between the angle at which resistance is felt in response to a slow velocity stretch (below that which would trigger the stretch reflex, R2) and that associated with a fast velocity stretch (as fast as possible, R1) to elicit a catch due to the stretch reflex was calculated. The two joint angles were measured using a goniometer centered over the lateral malleolus. The Tardieu angle (R2-R1) provides an indication of the severity of spasticity; with larger R2-R1 differences reflecting greater spasticity.

\section{Range of motion measures}

Active and passive range of motion was determined for both the affected and unaffected ankle joints, using a Biodex System 3 Dynamometer (Biodex Medical Systems Inc., Shirley, NY). The subject sat in the Biodex chair with their knee positioned at $120^{\circ}$ $\left(180^{\circ}=\right.$ full extension $)$ and their foot secured in a foot plate. Active range of motion was

determined as the total angular displacement achieved as the subject moved their ankle from maximum plantarflexion to maximum dorsiflexion. Similarly, passive range of motion was determined as the range (in degrees) that the experimenter was able to move the subject's ankle beginning in maximum plantarflexion, to maximum dorsiflexion. 


\section{Gait measures}

\section{Lab setup}

Kinematic and kinetic data were collected using two optoelectric cameras (Optotrak 3020, Northern Digital Inc., Waterloo, ON), placed on either side of an 8 metre long walkway instrumented with two embedded AMTI force platforms (Advanced Mechanical Technologies Inc., Newton, MA) (Figure 2). The Optotrak cameras recorded the spatial coordinates (X, Y and Z) of infrared light emitting diodes (IREDs). The force platforms recorded ground reaction forces in the $\mathrm{X}, \mathrm{Y}$ and $\mathrm{Z}$ directions. The Optotrak and force plate data were sampled at 100 and $200 \mathrm{~Hz}$, respectively and synchronized within the motion analysis software (C-Motion, Inc., Rockville, MD) during data processing. All equipment was calibrated and the location of the forceplates within the field of view was established prior to testing. This ensured the two Optotrak cameras were referenced to the same lab coordinate system and the force platforms were set to zero prior to data collection. 


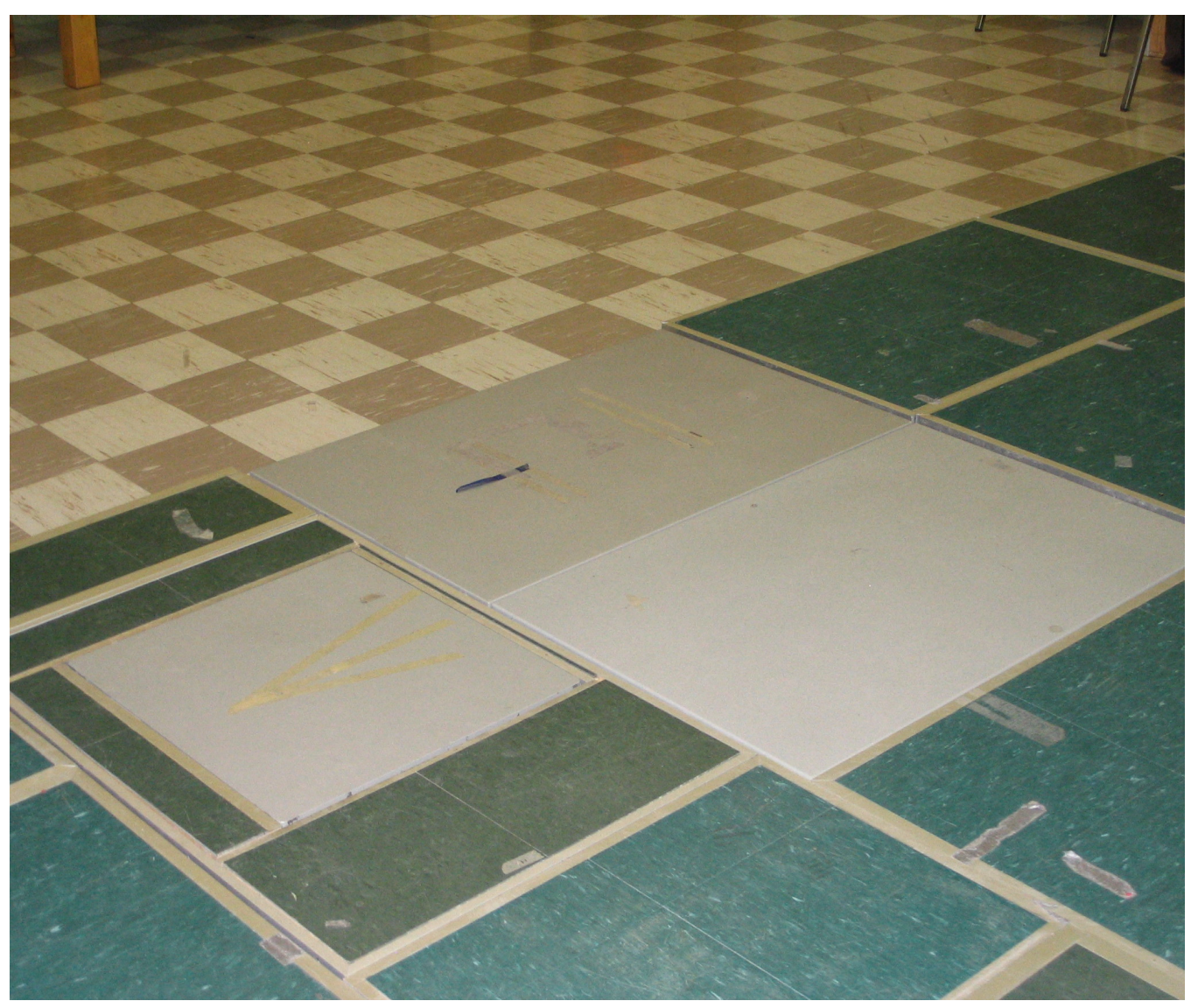

\section{Figure 2 Lab setup for gait testing}

\section{Procedures}

All data were collected in the Motor Performance Laboratory in the School of Rehabilitation Therapy at Queen's University, Kingston, Ontario. Ethics approval for the study was obtained from the Faculty of Health Science Research Ethics Board of Queen's University as well as the Providence Continuing Care Centre Ethics Review Board prior to subject recruitment.

Upon admission into the study, subjects were tested on two separate occasions approximately two weeks apart in order to establish stable baseline (B1 and B2) measurements. Intramuscular injections of BTX-A were then administered in a hospital 
setting by the attending physician (Appendix D). The BTX-A injection sites were determined by the physician based on a combination of visual gait assessment, active and passive range of motion of the ankle, and the Ashworth scale score assessing hypertonia of the plantarflexors. From the clinical impression, injections into any or all of the gastrocnemius (medial and lateral heads), soleus, tibialis posterior, flexor digitorum longus, and flexor hallucis longus muscles was considered. In cases where excessive inversion was present, tibialis anterior was also injected with BTX-A. All injections were guided using electromyography to localize the motor point and dosages delivered via a 26-gauge Teflon coated needle as per standard guidelines from the clinical recommendations of a botulinum toxin expert panel (Appendix E).

In order to assess the peak drug effect and the effect over time, testing was repeated at two weeks post-injection (T1), then at four week intervals after that (T2 and T3) for a total of five testing sessions and a study duration of twelve weeks. Signed informed consent was obtained at the start of the first baseline test session and a copy was provided for the subject's personal records.

Subjects reported to the laboratory wearing loose fitting shorts and shirt. All subjects wore the same pair of comfortable walking shoes at repeated test sessions. IREDs mounted on rigid plates in clusters of three or four were positioned and secured with over the top of the foot and secured with elasticized straps over the lateral aspects of the mid-shank and mid-thigh bilaterally. Clusters were also placed and secured over the pelvis and upper trunk. The clusters of four IRED markers arranged in a non collinear manner on the foot, thigh and pelvis and the clusters of three IRED markers (non 
collinear) on the shank and upper trunk were used to track lower limb and trunk motion during gait. A total of 29 IRED markers were used (Figure 3).

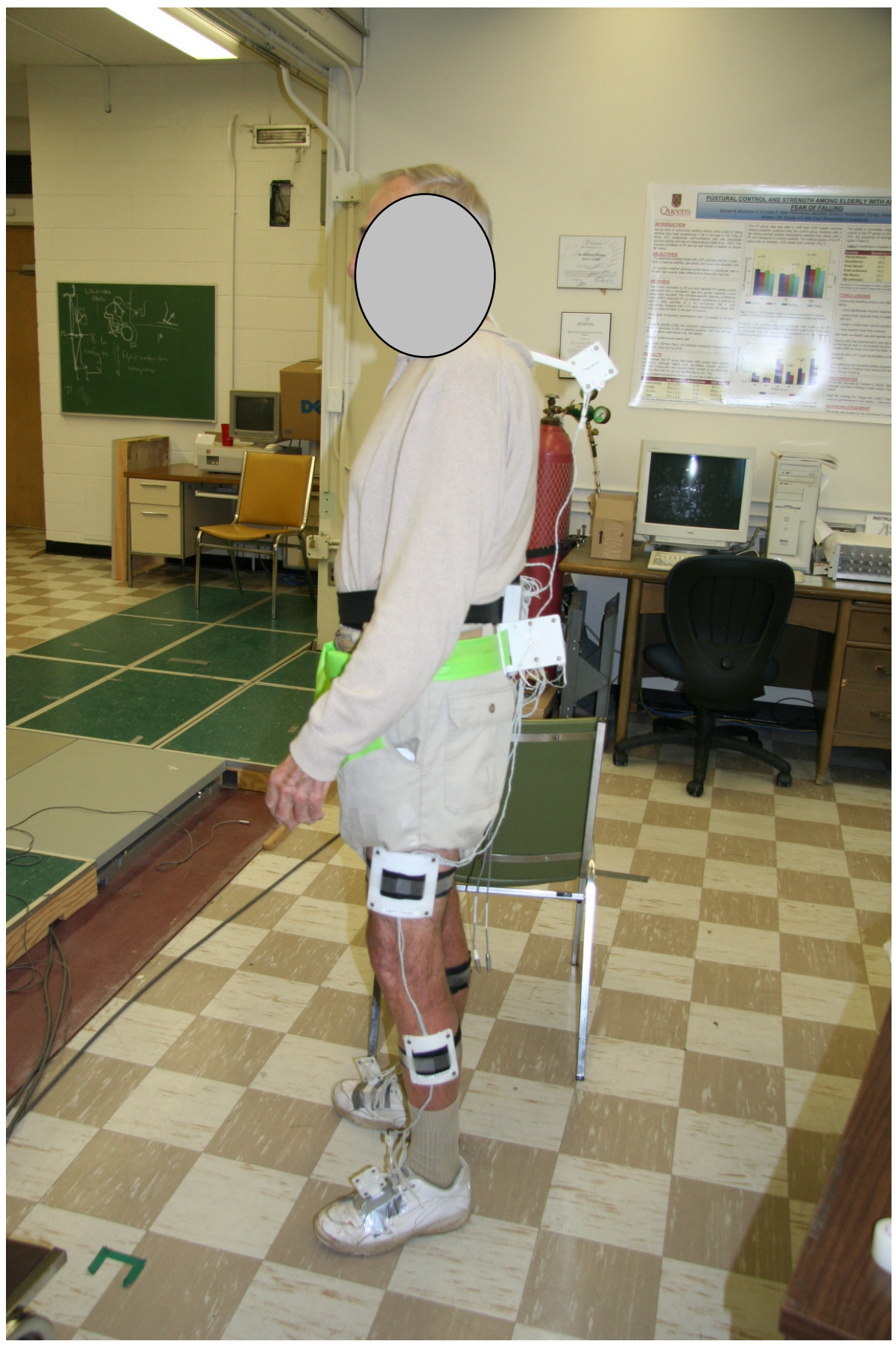

Figure 3 Standard configuration of infrared emitting diodes (IREDs) 
Once instrumented, subjects were instructed to walk along the walkway at their normal walking pace (i.e. self-selected walking speed) until three to five successful trials were acquired. A successful trial was one in which the subject landed with one foot on each forceplate and all IRED markers were visible by the cameras. Subjects did not use any assistive devices or ankle-foot orthoses during testing and all were given time to rest between trials as needed.

Following gait testing it was necessary to define the planes and axes about which motion occurred within the C-Motion motion analysis software. To provide the necessary reference information the tip of a pointed probe instrumented with four IRED markers was used to identify several virtual landmarks bilaterally. These probed landmarks approximated the first and fifth metatarsal heads, the medial and lateral malleoli, the medial and lateral epicondyles, the greater trochanter, a point aligned vertically with the greater trochanter at the level of the anterior superior iliac spine (ASIS), and the acromion process. The IRED markers on the probe were positioned at a fixed location with respect to the tip allowing the spatial coordinates of each virtual landmark to be calculated by transforming the IREDs' location to the tip of the probe (Figure 4). 


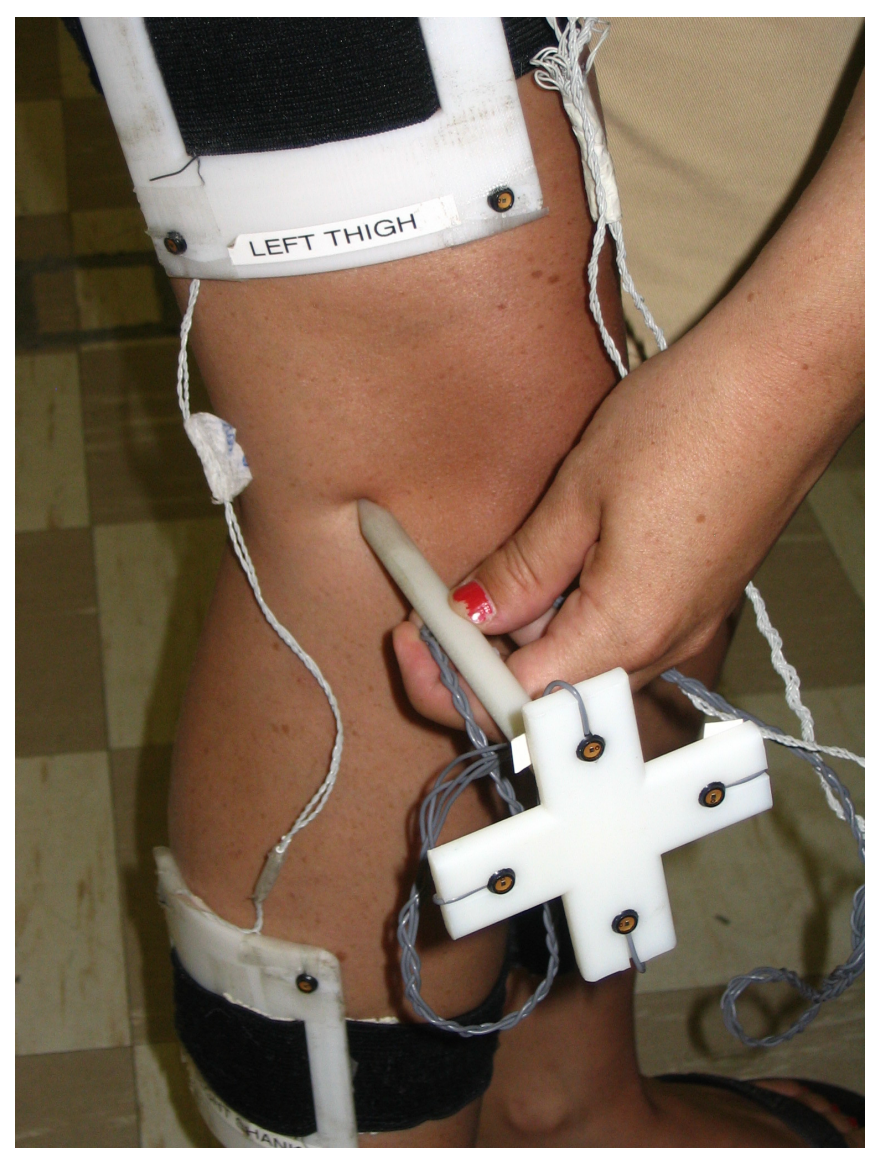

Figure 4 Illustration of the probe instrumented with infrared emitting diodes (IREDs), used to localize virtual landmarks

\section{Data processing}

A three dimensional (3-D) eight segment model was developed. Each segment was defined using the appropriate proximal and distal virtual markers identified with the probe and the marker cluster secured on that segment. The foot segment was defined by the foot cluster, the virtual first and fifth metatarsals and the virtual medial and lateral malleoli markers. The shank segment was defined by the shank cluster, the virtual medial and lateral malleoli and the virtual medial and lateral epicondyles markers. The thigh segment was defined by the thigh cluster, the virtual medial and lateral epicondyles and the virtual greater trochanter positions. The pelvis was defined by the pelvic cluster, the 
virtual greater trochanters and the virtual ASIS marker. Finally, the trunk was defined by the thoracic cluster, the virtual ASIS and the virtual acromion process markers. Within the computational software, a vertical axis about which the pelvis rotated was created by generating a virtual landmark at the level of the probe-defined ASIS landmark. The purpose of the vertical pelvic axis was to improve repeatability of the pelvic segment alignment over the five testing sessions for each subject as there is no suitable anatomical landmark. For the ankle and knee, the joint centre was calculated as the midpoint between the malleoli and epicondyles, respectively (Hamill and Selbie, 2004). For the hip, the joint centre was calculated as a point located at 0.25 times the distance between the two greater trochanter landmarks to the left or right as appropriate to the respective limb (Hamill and Selbie, 2004). Model anthropometrics were based on Dempster's table of segment masses and segment geometry for centres of mass and moments of inertia (Winter, 1987).

Gait events, including foot contact and toe off were identified for all trials. The baseline force recorded during the first 0.01 seconds of each trial (before the subject stepped on the forceplates) was averaged within C-Motion and the average value was then set as 0 Newtons to negate any offset. The foot contact and toe off were then labeled by visual inspection according to the point at which the vertical ground reaction force increased above baseline and decreased to baseline, respectively. Following this, the kinematic and kinetic profiles for the ankle, knee and hip were determined using inverse dynamic analysis for the stance phase (Winter, 1991). All data were filtered with a dual pass Butterworth filter with a cut-off frequency of $5 \mathrm{~Hz}$. All kinetic data were normalized to body mass and $100 \%$ of the stance phase of gait. Data from three to five trials for each 
subject were processed and joint angular displacement, moments and powers were calculated.

\section{Data analysis}

Data were processed for both sides to provide information about the affected and unaffected lower limbs throughout the stance phase of gait. Each outcome measure was calculated for individual trials and then averaged for each testing session. The baseline trials were consistent as evidenced by less than $5^{\circ}$ difference in maximum and minimum angular displacements at all joints in over $90 \%$ of cases (Appendix F). The baseline data were therefore averaged and served as the reference against which outcomes measured at T1, T2 and T3 were compared.

\section{Temporal spatial parameters}

The temporal-spatial parameters of gait speed $(\mathrm{m} / \mathrm{s})$, step length $(\mathrm{m})$, and stance time (s), were calculated from the kinematic data.

\section{Kinematic and kinetic parameters}

Maximum and minimum values as well as total range were calculated for joint angles (degrees) at the ankle, knee and hip for each trial. The total work (J/kg) absorbed and generated was determined as the negative and positive area under the power curves, respectively. BTX-A was expected to exert a direct effect on the ankle plantarflexors and therefore, it was also of interest to document the ankle angle at initial contact and the 
power associated with push off, defined as the maximum ankle power occurring prior to the end of stance phase.

\section{Individual subject data}

Due to the intersubject variability in gait, the kinematic and kinetic measures for individual subjects were described on a case by case basis and according to treatment responsiveness ${ }^{1}$ in order to describe the short term (T1) and longer term (T3) effects of BTX-A on gait. T1 is the point in time associated with the peak effect of the BTX-A and T3 reveals the sustainability of any effect.

\section{Statistical analysis}

The significance of changes in dependent measures over time was determined using an analysis of variance for repeated measures (Baseline, T1, T2 and T3), and a Least Significant Difference (LSD) post hoc analysis was used as appropriate to establish between which time points the differences lay. The significance of the differences between active and passive range of motion at baseline was determined using a paired samples T-test. A probability of $\mathrm{p}<0.05$ was adopted for all analyses.

\footnotetext{
${ }^{1}$ Responsiveness was determined on the basis of clinical measures. A reduction in Ashworth score accompanied by an increase in passive range of motion after BTX-A injection was indicative of a positive response (Burbaud et al., 1996; Hesse et al., 1994; Pierson et al., 1996; Reiter et al., 1998)
} 


\section{CHAPTER 4}

\section{RESULTS}

\section{Subject demographics}

A total of seven subjects (2 females, 5 males) with chronic stroke were recruited for this study. The range and mean age of the subjects were 20 to 83 years and 63.1 years, respectively. Three subjects presented with left hemiparesis and four subjects presented with right hemiparesis. The subjects reported having had their strokes an average of 5 years and 5 months prior to baseline testing, the most recent being 10 months and the longest being 14 years. Demographic and stroke characteristics are presented in Table 1.

Table 1 Summary of subject demographics and stroke characteristics

\begin{tabular}{ccccccc}
\hline Subject & Sex & $\begin{array}{c}\text { Age } \\
\text { (years) }\end{array}$ & $\begin{array}{c}\text { Weight } \\
(\mathbf{k g})\end{array}$ & $\begin{array}{c}\text { Height } \\
(\mathbf{m})\end{array}$ & $\begin{array}{c}\text { Time since } \\
\text { stroke } \\
(\mathbf{m o n t h})\end{array}$ & $\begin{array}{c}\text { Side } \\
\text { affected }\end{array}$ \\
\hline $\mathbf{1}$ & $\mathrm{M}$ & 83 & 80 & 1.78 & 61 & $\mathrm{~L}$ \\
$\mathbf{2}$ & $\mathrm{F}$ & 49 & 72 & 1.79 & 29 & $\mathrm{R}$ \\
$\mathbf{3}$ & $\mathrm{M}$ & 72 & 93 & 1.70 & 169 & $\mathrm{~L}$ \\
$\mathbf{4}$ & $\mathrm{F}$ & 65 & 75 & 1.60 & 155 & $\mathrm{R}$ \\
$\mathbf{5}$ & $\mathrm{M}$ & 80 & 75 & 1.84 & 12 & $\mathrm{R}$ \\
$\mathbf{6}$ & $\mathrm{M}$ & 20 & 90 & 1.90 & 10 & $\mathrm{R}$ \\
$\mathbf{7}$ & $\mathrm{M}$ & 73 & 56 & 1.57 & 19 & $\mathrm{~L}$ \\
\hline Mean & & 63.1 & 77.3 & 174 & 65 & \\
SD & & 22.0 & 12.3 & 12.2 & 68.5 & \\
\hline
\end{tabular}




\section{Clinical measures}

Clinical measures at baseline for individual subjects are presented in Table 2. Measures are reported as the average of baseline 1 (B1) and baseline 2 (B2). All subjects entered the study with moderate to severe ankle hypertonicity as determined by the Modified Ashworth Scale (MAS) (scores $\geq 3$ ). Spasticity, according to the Tardieu scale, was indicated by the presence of ankle clonus (Yes or No) and the difference between the angles at which resistance is felt during rapid dorsiflexion (R1) and passive dorsiflexion end range (R2). Three of the seven subjects had clonus and R2-R1 differences exceeding $10^{\circ}$ as indicated by the Tardieu scale at baseline. Active range of motion (AROM) at the ankle was significantly less than passive range of motion (PROM) on the affected side $(\mathrm{p}<0.001)$ and the unaffected side $(\mathrm{p}<0.001) . \quad A R O M$ and PROM were significantly less on the affected side when compared to the unaffected side $(\mathrm{p}<0.001)$. In combination these findings reflect both weakness and elevated muscle tone restricting joint mobility. The expected PROM and AROM in healthy individuals is about $70^{\circ}$ from full plantarflexion to full dorsiflexion (Hoppenfeld, 1976); PROM values less than this suggests the presence of contracture and large differences between PROM and AROM are indicative of weakness as the muscles are unable to generate the force needed to move the limb through the full range. In this study, all subjects presented with ankle weakness as indicated by the large difference between the AROM and PROM, where subject 3 appears to be the weakest, and subjects 1, 4 and 5 appear to have the strongest ankle muscles (Table 2). 
Table 2 Summary of clinical measures at baseline

\begin{tabular}{|c|c|c|c|c|c|c|c|c|c|}
\hline \multirow[t]{2}{*}{ Subject } & \multicolumn{2}{|c|}{$\operatorname{AROM}\left(^{\circ}\right)$} & \multicolumn{2}{|c|}{$\operatorname{PROM}\left(^{\circ}\right)$} & \multicolumn{2}{|c|}{$\begin{array}{c}\text { Difference } \\
\text { between } \\
\text { AROM \& } \\
\left.\text { PROM ( }{ }^{\circ}\right)\end{array}$} & \multirow[t]{2}{*}{$\begin{array}{l}\text { MAS } \\
\text { Score }\end{array}$} & \multirow[t]{2}{*}{$\begin{array}{c}\text { Clonus } \\
(\mathbf{Y} \text { or } \mathbf{N})\end{array}$} & \multirow[t]{2}{*}{$\begin{array}{c}\text { Tardieu } \\
\text { Angle }\left(^{\circ}\right) \\
\text { R2-R1 }\end{array}$} \\
\hline & $\mathbf{A}$ & UA & $\mathbf{A}$ & $\mathbf{U A}$ & $\mathbf{A}$ & UA & & & \\
\hline 1 & 37.0 & 59.0 & 56.0 & 68.5 & 19.0 & 9.5 & 3 & $Y$ & 13.0 \\
\hline 2 & 24.0 & 50.5 & 48.5 & 68.0 & 26.5 & 19.5 & 3 & $\mathrm{Y}$ & 15.5 \\
\hline 3 & 11.5 & 56.5 & 62.0 & 74.5 & 50.5 & 18.0 & 4 & $\mathrm{~N}$ & 6.0 \\
\hline 4 & 21.0 & 53.0 & 59.0 & 73.0 & 16.0 & 20.0 & 3 & $\mathrm{~N}$ & 6.5 \\
\hline 5 & 31.0 & 48.0 & 48.5 & 62.0 & 17.5 & 18.0 & 3 & $\mathrm{~N}$ & 1.5 \\
\hline 6 & 17.5 & 48.0 & 49.5 & 62.5 & 32.0 & 14.5 & 4 & $\mathrm{~N}$ & 4.5 \\
\hline 7 & 66 & 87.5 & 95.5 & 107.5 & 29.5 & 20.0 & 3 & $\mathrm{Y}$ & 12.0 \\
\hline Mean & $29.7 * \dagger$ & $57.5^{\dagger \mathrm{b}}$ & $59.6^{*^{\mathrm{a}}}$ & $73.7^{\mathrm{ab}}$ & 27.3 & 17.1 & & & 8.4 \\
\hline SD & 18.1 & 13.9 & 16.6 & 15.6 & 12.0 & 3.8 & & & 5.1 \\
\hline $\begin{array}{l}\mathrm{A}=\text { Aff } \\
\text { passive } \\
\text { slow stre } \\
* \dagger \mathrm{ab} p\end{array}$ & $\begin{array}{l}\text { cted sid } \\
\text { nge of } r \\
\text { ch and } r \\
0.05\end{array}$ & $\mathrm{UA}=$ & Unaffec & ted side & $\begin{array}{l}\text { ARO } \\
\text { Ashw } \\
\text { antarl }\end{array}$ & $\begin{array}{l}=\mathrm{A} \\
\mathrm{h} \mathrm{Sc} \\
\text { xors }\end{array}$ & ive ran & $\begin{array}{l}\text { of motic } \\
=\text { Differe }\end{array}$ & $\begin{array}{l}\text { PROM = } \\
\text { e between }\end{array}$ \\
\hline
\end{tabular}

In order to classify "responders" and "non-responders" to the BTX-A treatment the changes observed in clinical measures on the affected side two weeks post-injection (T1) were considered (Table 3). Ankle clonus was no longer present in subject 1 and subject 7 two weeks post-injection (T1). Fatigable clonus (less than 10 seconds) was still present in subject 2 at T1. In terms of PROM, subjects 1, 5, and 6 demonstrated increases greater than $7^{\circ}$ at $\mathrm{T} 1$. Subjects $2,3,4$, and 7 did not show any appreciable increase in PROM at T1 or showed a slight decrease in PROM. Subjects 3, 5, and 6 achieved greater AROM at $\mathrm{T} 1\left(\geq 6^{\circ}\right)$. Based on the overall clinical profiles at T1, subjects 2 and 4 were classified as "non-responders" to the BTX-A treatment and subject 1, 5 and 6 were clear “responders". Subjects 3 and 7 had mixed clinical findings; however, based on reductions in MAS paired with the resolution of clonus (subject 7) or marked gain in AROM (subject 3) both were categorized as "responders". 
Table 3 Summary of clinical measures obtained on the affected side two weeks post BTX-A injection (T1)

\begin{tabular}{|c|c|c|c|c|c|c|c|c|c|}
\hline \multirow[t]{2}{*}{ Subject } & \multicolumn{3}{|c|}{$\operatorname{AROM}\left(^{\circ}\right)$} & \multicolumn{3}{|c|}{$\operatorname{PROM}\left(^{\circ}\right)$} & \multicolumn{2}{|c|}{$\begin{array}{c}\text { Tardieu } \\
\text { Clonus } \\
(\mathbf{Y} \text { or } \mathbf{N})\end{array}$} & \multirow[t]{2}{*}{$\begin{array}{c}\text { MAS } \\
\Delta\end{array}$} \\
\hline & B & T1 & $\Delta$ & B & T1 & $\Delta$ & B & T1 & \\
\hline 1 & 37.0 & 39.0 & 2.0 & 56.0 & 65.0 & 9.0 & $\mathrm{Y}$ & $\mathrm{N}$ & 0 \\
\hline 2 & 24.0 & 18.0 & -6.0 & 48.5 & 45.0 & -3.5 & $\mathrm{Y}$ & $\mathrm{Y}$ & 0 \\
\hline 3 & 11.5 & 20.0 & 8.5 & 62.0 & 59.0 & -3.0 & $\mathrm{~N}$ & $\mathrm{~N}$ & -1 \\
\hline 4 & 21.0 & 17.0 & -4.0 & 59.0 & 60.0 & 1.0 & $\mathrm{~N}$ & $\mathrm{~N}$ & 0 \\
\hline 5 & 31.0 & 37.0 & 6.0 & 48.5 & 56.0 & 7.5 & $\mathrm{~N}$ & $\mathrm{~N}$ & -1 \\
\hline 6 & 17.5 & 30.0 & 12.5 & 49.5 & 68.0 & 18.5 & $\mathrm{~N}$ & $\mathrm{~N}$ & -1 \\
\hline 7 & 66.0 & 68.0 & 2.0 & 95.5 & 91.0 & -4.5 & $\mathrm{Y}$ & $\mathrm{N}$ & -1 \\
\hline Mean & 29.7 & 32.7 & & 59.6 & 63.4 & & & & \\
\hline SD & 18.1 & 18.0 & & 16.6 & 14.2 & & & & \\
\hline
\end{tabular}

$\mathrm{AROM}=$ Active range of motion, $\mathrm{PROM}=$ Passive range of motion; MAS $\Delta=$ difference between Modified Ashworth Scale score at T1 and baseline; B = Average baseline; $\mathrm{T} 1=$ Two weeks post BTX-A injection; $\Delta=$ difference between $\mathrm{T} 1$ and Baseline values.

Shaded rows identify clinically defined "non-responders".

\section{Gait measures}

\section{Temporal distal parameters}

Individual subject temporal distal data are provided in Table 4, with the clinically defined "non-responders" identified. Clinically, subjects 2 and 4 were characterized as "non-responders" yet they were not distinguishable from the other subjects in terms of changes in gait speed or the temporal distance parameters following BTX-A treatment. With the exception of subject 1 who more than tripled his gait speed, all others showed modest or no change relative to baseline. Mean and standard deviations for temporal distal parameters of gait over time relative to BTX-A treatment are listed in Table 5. No significant differences were found for any measures across time on the affected side ( $p>0.240)$, unaffected side $(p>0.489)$, or in terms of interlimb symmetry (ratio of the affected to unaffected side) $(\mathrm{p}>0.193)$. 
Table 4 Individual subject step length (m), stance time (sec) and gait speed (m/s) at baseline and post-injection (T1, T2, and T3)

\begin{tabular}{|c|c|c|c|c|c|c|c|c|c|c|c|c|c|}
\hline & \multirow[t]{2}{*}{ Subject } & \multicolumn{3}{|c|}{ Baseline } & \multicolumn{3}{|c|}{$\begin{array}{c}\text { T1 } \\
\text { (2 weeks post-injection) }\end{array}$} & \multicolumn{3}{|c|}{$\begin{array}{c}\mathrm{T} 2 \\
\text { (6 weeks post-injection) }\end{array}$} & \multicolumn{3}{|c|}{$\begin{array}{c}\mathrm{T3} \\
\text { (10 weeks post-injection) }\end{array}$} \\
\hline & & $\mathbf{A}$ & UA & A:UA & A & UA & A:UA & A & UA & A:UA & A & UA & A:UA \\
\hline \multirow{7}{*}{$\begin{array}{c}\text { Step } \\
\text { length } \\
(\mathbf{m})\end{array}$} & 1 & 0.16 & 0.09 & 1.85 & 0.26 & 0.14 & 1.83 & 0.26 & 0.14 & 1.83 & 0.33 & 0.17 & 1.96 \\
\hline & 2 & 0.65 & 0.48 & 1.37 & 0.62 & 0.52 & 1.21 & 0.66 & 0.50 & 1.32 & 0.66 & 0.51 & 1.29 \\
\hline & 3 & 0.42 & 0.19 & 2.23 & 0.41 & 0.25 & 1.66 & 0.42 & 0.24 & 1.76 & 0.44 & 0.14 & 3.10 \\
\hline & 4 & 0.43 & 0.22 & 1.94 & 0.40 & 0.27 & 1.48 & 0.39 & 0.32 & 1.19 & 0.37 & 0.29 & 1.29 \\
\hline & 5 & 0.58 & 0.48 & 1.20 & 0.59 & 0.40 & 1.47 & 0.53 & 0.43 & 1.21 & 0.52 & 0.42 & 1.24 \\
\hline & 6 & 0.62 & 0.42 & 1.48 & 0.65 & 0.44 & 1.50 & 0.63 & 0.44 & 1.42 & 0.61 & 0.47 & 1.30 \\
\hline & 7 & 0.28 & 0.17 & 1.63 & 0.25 & 0.15 & 1.68 & 0.27 & 0.10 & 2.64 & 0.28 & 0.16 & 1.73 \\
\hline \multirow{7}{*}{$\begin{array}{c}\text { Stance } \\
\text { time } \\
\text { (sec) }\end{array}$} & 1 & 2.03 & 1.94 & 1.05 & 1.17 & 1.24 & 0.94 & 0.83 & 0.93 & 0.89 & 0.87 & 1.01 & 0.86 \\
\hline & 2 & 1.26 & 1.43 & 0.88 & 1.05 & 1.41 & 0.74 & 1.23 & 1.41 & 0.87 & 1.15 & 1.35 & 0.85 \\
\hline & 3 & 1.62 & 2.01 & 0.81 & 2.00 & 2.44 & 0.82 & 1.79 & 2.23 & 0.80 & 1.81 & 2.28 & 0.79 \\
\hline & 4 & 0.94 & 1.21 & 0.77 & 0.90 & 1.15 & 0.78 & 0.98 & 1.14 & 0.86 & 1.01 & 1.26 & 0.80 \\
\hline & 5 & 0.92 & 1.04 & 0.88 & 0.91 & 1.08 & 0.84 & 0.94 & 1.07 & 0.88 & 0.86 & 1.10 & 0.78 \\
\hline & 6 & 0.95 & 1.09 & 0.88 & 0.88 & 1.09 & 0.81 & 0.88 & 1.09 & 0.81 & 0.99 & 1.14 & 0.87 \\
\hline & 7 & 1.00 & 1.31 & 0.77 & 1.05 & 1.39 & 0.76 & 0.97 & 1.29 & 0.75 & 0.98 & 1.29 & 0.76 \\
\hline \multirow{7}{*}{$\begin{array}{c}\text { Gait } \\
\text { speed } \\
(\mathbf{m} / \mathbf{s})\end{array}$} & 1 & \multicolumn{3}{|c|}{0.12} & \multicolumn{3}{|c|}{0.27} & \multicolumn{3}{|c|}{0.33} & \multicolumn{3}{|c|}{0.40} \\
\hline & 2 & \multicolumn{3}{|c|}{0.56} & \multicolumn{3}{|c|}{0.59} & \multicolumn{3}{|c|}{0.60} & \multicolumn{3}{|c|}{0.63} \\
\hline & 3 & \multicolumn{3}{|c|}{0.25} & \multicolumn{3}{|c|}{0.23} & \multicolumn{3}{|c|}{0.25} & \multicolumn{3}{|c|}{0.23} \\
\hline & 4 & \multicolumn{3}{|c|}{0.41} & \multicolumn{3}{|c|}{0.43} & \multicolumn{3}{|c|}{0.45} & \multicolumn{3}{|c|}{0.41} \\
\hline & 5 & \multicolumn{3}{|c|}{0.74} & \multicolumn{3}{|c|}{0.69} & \multicolumn{3}{|c|}{0.64} & \multicolumn{3}{|c|}{0.60} \\
\hline & 6 & \multicolumn{3}{|c|}{0.71} & \multicolumn{3}{|c|}{0.73} & \multicolumn{3}{|c|}{0.72} & & 0.70 & \\
\hline & 7 & & 0.29 & & & 0.24 & & & 0.24 & & & 0.28 & \\
\hline
\end{tabular}

$\mathrm{A}=$ Affected side; $\overline{\mathrm{UA}}=$ Unaffected side; $\mathrm{A}: \mathrm{UA}=$ Ratio of affected to unaffected side; $\mathrm{T} 1$ = Two weeks post-injection; $\mathrm{T} 2$ = Six weeks post-injection; T3 = Ten weeks post-injection 
Table 5 Mean and standard deviations of temporal spatial parameters of gait at baseline, T1, T2 and T3

\begin{tabular}{|c|c|c|c|c|c|}
\hline Parameter & Side & Baseline & T1 & $\mathbf{T} 2$ & T3 \\
\hline Speed $(\mathrm{m} / \mathrm{s})$ & & $0.44 \pm 0.24$ & $0.45 \pm 0.22$ & $0.46 \pm 0.20$ & $0.46 \pm 0.18$ \\
\hline \multirow{3}{*}{$\begin{array}{l}\text { Step length } \\
\text { (m) }\end{array}$} & $\mathbf{A}$ & $0.45 \pm 0.18$ & $0.45 \pm 0.17$ & $0.45 \pm 0.16$ & $0.46 \pm 0.14$ \\
\hline & UA & $0.29 \pm 0.16$ & $0.31 \pm 0.14$ & $0.31 \pm 0.16$ & $0.31 \pm 0.16$ \\
\hline & A:UA & $1.67 \pm 0.36$ & $1.55 \pm 0.20$ & $1.62 \pm 0.51$ & $1.70 \pm 0.67$ \\
\hline \multirow{3}{*}{$\begin{array}{l}\text { Stance time } \\
\quad(\mathrm{sec})\end{array}$} & $\mathbf{A}$ & $1.24 \pm 0.43$ & $1.14 \pm 0.39$ & $1.09 \pm 0.33$ & $1.10 \pm 0.33$ \\
\hline & UA & $1.43 \pm 0.39$ & $1.40 \pm 0.48$ & $1.31 \pm 0.44$ & $1.35 \pm 0.43$ \\
\hline & A:UA & $0.86 \pm 0.10$ & $0.81 \pm 0.07$ & $0.84 \pm 0.05$ & $0.82 \pm 0.04$ \\
\hline
\end{tabular}

\section{Kinematic measures}

Mean and standard deviations for the kinematic measures of gait are presented in Table 6 for baseline and post-injection trials. As a group, significant changes over time were detected at the ankle $(\mathrm{p}<0.05)$. Relative to baseline, there was a significant increase in the maximum dorsiflexion angle achieved at $\mathrm{T} 3$ (10 weeks post-injection) on the affected side $(\mathrm{p}=0.032)$. Subjects also demonstrated decreased maximum plantarflexion at T3 compared to baseline, T1 and T2 $(\mathrm{p}<0.05)$. As well, at T3 the ankle position at initial contact was in less plantarflexion $(\mathrm{p}=0.028)$ than occurred at baseline; the normal being approximately neutral. These changes associated with the treated ankle were not accompanied by any alterations of the unaffected ankle ( $p>0.484)$, or changes at the knee $(\mathrm{p}>0.244)$ or hip $(\mathrm{p}>0.164)$ of either side (Table 6). Figure 5 illustrates an example of the kinematic profiles at baseline, T1, T2 and T3 of a "responder" and "non- 
responder" (as classified by clinical measures) to the BTX-A treatment. Kinematic profiles for individual subjects are found in Appendix G.

Table 6 Mean and standard deviations of kinematic measures of gait during stance at baseline and post-injection (T1, T2 and T3)

\begin{tabular}{|c|c|c|c|c|c|}
\hline Parameter & Side & Baseline & T1 & $\mathbf{T 2}$ & T3 \\
\hline \multirow{2}{*}{$\operatorname{MaxDF}\left({ }^{\circ}\right)$} & $\mathbf{A}$ & $4.61 \pm 5.78^{*}$ & $5.53 \pm 6.05$ & $5.47 \pm 6.11$ & $7.38 \pm 5.68 *$ \\
\hline & $\mathbf{U A}$ & $21.21 \pm 6.67$ & $20.35 \pm 6.94$ & $20.78 \pm 5.44$ & $19.89 \pm 5.59$ \\
\hline \multirow{2}{*}{$\operatorname{MaxPF}\left({ }^{\circ}\right)$} & $\mathbf{A}$ & $-13.79 \pm 2.89$ & $-14.24 \pm 3.61$ & $-13.03 \pm 2.34$ & $-11.18 \pm 3.68 *$ \\
\hline & UA & $-10.75 \pm 9.43$ & $-9.97 \pm 8.73$ & $-10.45 \pm 8.20$ & $-10.36 \pm 7.09$ \\
\hline \multirow{2}{*}{$\begin{array}{c}\text { Ankle range } \\
\left({ }^{\circ}\right)\end{array}$} & $\mathbf{A}$ & $18.40 \pm 4.82$ & $19.78 \pm 4.24$ & $18.49 \pm 4.65$ & $18.56 \pm 3.17$ \\
\hline & UA & $31.96 \pm 10.85$ & $30.32 \pm 8.38$ & $31.22 \pm 10.26$ & $30.24 \pm 8.20$ \\
\hline \multirow{2}{*}{$\begin{array}{c}\text { Knee } \\
\text { MaxFlex }\left(^{\circ}\right)\end{array}$} & $\mathbf{A}$ & $20.69 \pm 13.97$ & $21.41 \pm 16.85$ & $22.69 \pm 13.08$ & $25.57 \pm 12.82$ \\
\hline & UA & $50.47 \pm 10.03$ & $49.62 \pm 10.61$ & $48.68 \pm 10.58$ & $51.23 \pm 10.00$ \\
\hline \multirow{2}{*}{$\begin{array}{c}\text { Knee } \\
\operatorname{MaxExt}\left({ }^{\circ}\right)\end{array}$} & $\mathbf{A}$ & $-6.84 \pm 7.76$ & $-5.61 \pm 7.58$ & $-5.63 \pm 7.55$ & $-3.85 \pm 6.19$ \\
\hline & UA & $7.90 \pm 7.81$ & $7.56 \pm 6.21$ & $4.99 \pm 8.97$ & $7.18 \pm 9.84$ \\
\hline \multirow{2}{*}{$\begin{array}{c}\text { Knee range } \\
\left({ }^{\circ}\right)\end{array}$} & $\mathbf{A}$ & $27.53 \pm 8.74$ & $27.02 \pm 10.87$ & $28.31 \pm 8.50$ & $29.42 \pm 8.42$ \\
\hline & $\mathbf{U A}$ & $42.57 \pm 7.30$ & $42.06 \pm 8.13$ & $43.69 \pm 4.73$ & $44.05 \pm 3.37$ \\
\hline \multirow{2}{*}{$\frac{\text { Hip }}{\text { MaxFlex }\left(^{\circ}\right)}$} & $\mathbf{A}$ & $15.27 \pm 4.18$ & $14.94 \pm 6.53$ & $15.34 \pm 6.36$ & $13.01 \pm 6.46$ \\
\hline & UA & $26.83 \pm 6.78$ & $25.66 \pm 7.52$ & $23.95 \pm 8.14$ & $26.34 \pm 10.51$ \\
\hline \multirow{2}{*}{$\underset{\operatorname{MaxExt}}{\operatorname{Hip}}$} & $\mathbf{A}$ & $-1.73 \pm 5.70$ & $-2.28 \pm 7.33$ & $-2.07 \pm 5.21$ & $-2.50 \pm 6.26$ \\
\hline & $\mathbf{U A}$ & $-7.05 \pm 8.60$ & $-8.07 \pm 6.72$ & $-9.42 \pm 5.28$ & $-11.44 \pm 7.18$ \\
\hline \multirow{2}{*}{$\begin{array}{c}\text { Hip range } \\
\left({ }^{\circ}\right)\end{array}$} & $\mathbf{A}$ & $17.00 \pm 6.98$ & $17.21 \pm 7.70$ & $17.41 \pm 6.64$ & $15.51 \pm 6.67$ \\
\hline & UA & $33.88 \pm 9.59$ & $33.73 \pm 7.28$ & $33.37 \pm 7.45$ & $37.78 \pm 6.60$ \\
\hline \multirow{2}{*}{ Ankle IC $\left(^{\circ}\right)$} & $\mathbf{A}$ & $-9.81 \pm 3.98 *$ & $-8.41 \pm 4.97$ & $-8.60 \pm 4.76$ & $-7.03 \pm 5.04^{*}$ \\
\hline & UA & $2.97 \pm 3.41$ & $2.60 \pm 3.28$ & $3.02 \pm 2.85$ & $4.29 \pm 3.01$ \\
\hline
\end{tabular}

$\mathrm{A}=$ Affected side; $\mathrm{UA}=$ Unaffected side; $\mathrm{MaxDF}=\mathrm{Max}$ imum dorsiflexion angle; MaxPF = Maximum plantarflexion angle; MaxFlex= Maximum flexion angle; MaxExt = Maximum extension angle; Ankle IC = Ankle angle at initial contact; T1 = Two weeks post injection; T2 = Six weeks post-injection; T3 = Ten weeks post-injection $* \mathrm{p}<0.05$ 

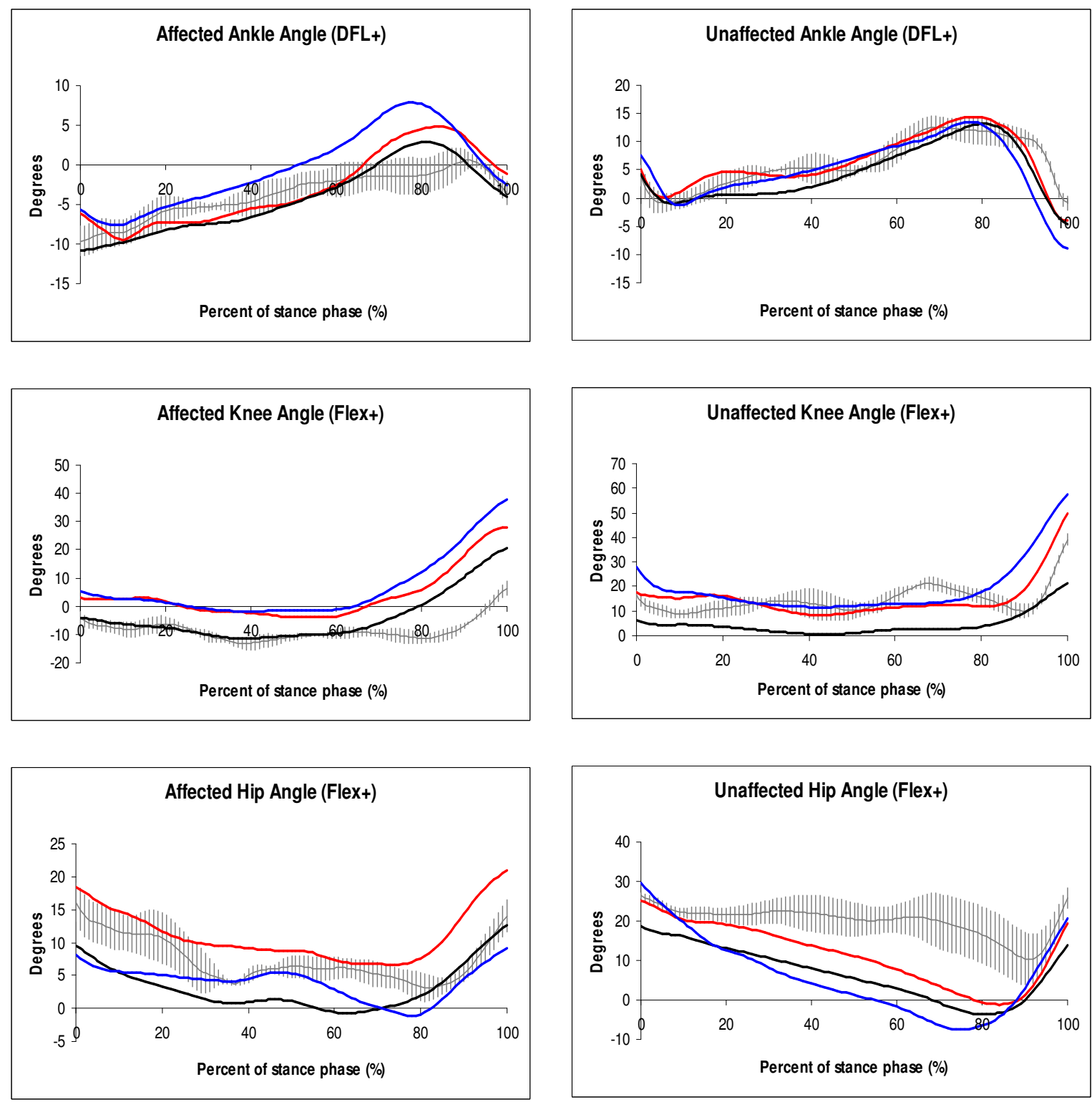

Figure 5a Kinematics at the ankle, knee and hip of a "responder"(subject 1) at Baseline (light grey), T1 (red), T2 (black) and T3 (blue). Shaded area reflects the standard deviation of the two baseline measures. Note the different scales on Y-axis for the affected and unaffected sides. 

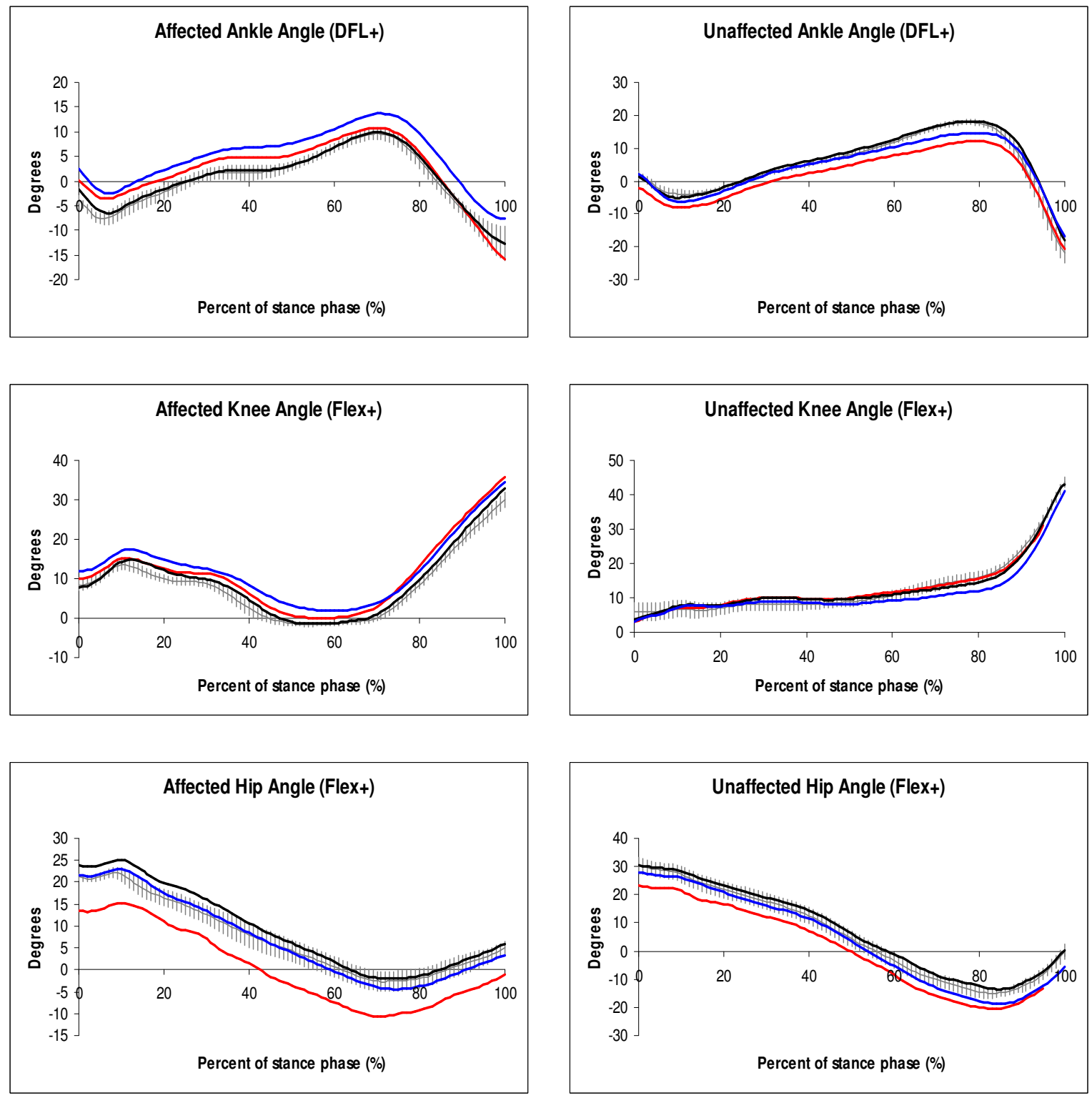

Figure 5b Kinematics at the ankle, knee and hip of a "non-responder"(subject 2) at Baseline (light grey), T1 (red), T2 (black) and T3 (blue). Shaded area reflects the standard deviation of the two baseline measures. Note the different scales on Y-axis for the affected and unaffected sides. 
Examination of individual subject's data revealed that all but subject 7 showed gains in maximum dorsiflexion which were sustained to $\mathrm{T} 3$, though to a variable extent $\left(1^{\circ}\right.$ to $\left.7^{\circ}\right)$. The amount of plantarflexion peak angles tended to be less in all subjects with peak angles lower by $2^{\circ}$ to $5^{\circ}$ in 5 out of 7 subjects and by less than $1^{\circ}$ in the other 2 subjects (subjects 3 and 7). Consistent with this finding was that most subjects (except subject 5) contacted the ground with their affected foot in less plantarflexion 10 weeks following BTX-A treatment. In fact, one subject (subject 2) achieved initial contact in dorsiflexion, something that none could do at baseline.

\section{Power and work measures}

Means and standard deviations of the peak power generated and absorbed at the ankle and total work performed at each joint during walking, calculated relative to the BTX-A treatment, are presented in Table 7. No changes in peak power generation or absorption at the ankle or total positive or negative work on either the affected or unaffected side at any joint were detected $(\mathrm{p} \geq 0.148)$. In terms of work (area above/below the power curves), less negative work was performed by the eccentrically contracting plantarflexors on the unaffected side 2 weeks following BTX-A injection (T1). This was associated with increased symmetry in the peak power absorbed and total negative work performed by the plantarflexors at $\mathrm{T} 1$ relative to baseline $(\mathrm{p}=0.038$ and $\mathrm{p}=0.027$, respectively). This pattern was not sustained beyond 2 weeks post treatment. Of note is that the changes in ankle kinetics were accompanied by progressively greater symmetry of the total work performed by the hip at $\mathrm{T} 1$ and $\mathrm{T} 2$, which reached significance relative to baseline $(\mathrm{p}=0.040)$. Again, this was not sustained. Because of the high variability in 
the data between subjects, change scores relative to baseline were also calculated and analyzed with a repeated measures ANOVA. This failed to identify any changes not already described above.

Examination of the individual data revealed that only 3 of the 7 subjects were able to perform greater work using the plantarflexors at T3, yet 5 of the 7 subjects generated more power from early concentric extension of the hip and later the hip flexors. Two of those subjects showed increases in both plantarflexor and total positive hip work performed (subjects 1 and 2), whereas the other 3 (subjects 3, 6 and 7) showed gains in positive work performed by the hip extensors and flexors but a loss in total plantarflexor work done.

Figure 6 illustrates the power profiles for each joint over time of a clinically defined "responder" (a) and "non responder" (b). The "non-responder" (Figure 6b), in a manner similar to the "responder" (Figure 6a), shows a trend toward improved ankle power kinetics post-injection, but not to the same extent as the responder.

It was expected that normalizing tone in the plantarflexors and promoting greater ankle mobility through BTX-A treatment would result improvements in gait kinematics and kinetics. Although some subjects showed marked improvements in some parameters there was not a readily identifiable clinical profile of those subjects who would respond best in terms of gait performance. However, all reported they were satisfied with the treatment. A summary of the clinical and gait changes observed early post-treatment (T1) and longer term (T3, 10 weeks post-treatment) relative to baseline is presented in Table 8 . 
Table 7 Mean and standard deviations of power and work measures at the ankle and hip during stance at Baseline and post-injection (T1, T2 and T3)

\begin{tabular}{|c|c|c|c|c|c|}
\hline Parameter & Side & Baseline & T1 & T2 & T3 \\
\hline \multirow{5}{*}{$\begin{array}{l}\text { Ankle Max } \\
\text { Gen (W/kg) }\end{array}$} & A & $0.40 \pm 0.38$ & $0.43 \pm 0.44$ & $0.42 \pm 0.42$ & $0.41 \pm 0.39$ \\
\hline & UA & $1.82 \pm 0.75$ & $1.36 \pm 1.11$ & $1.75 \pm 0.72$ & $1.78 \pm 0.96$ \\
\hline & UA-A & $1.42 \pm 0.51$ & $1.63 \pm 1.00$ & $1.33 \pm 0.44$ & $1.37 \pm 0.72$ \\
\hline & $\Delta \mathbf{A}$ & - & $-0.02 \pm 0.16$ & $0.02 \pm 0.17$ & $0.01 \pm 0.18$ \\
\hline & $\Delta \mathbf{U A}$ & - & $-0.17 \pm 0.35$ & $-0.07 \pm 0.22$ & $-0.04 \pm 0.55$ \\
\hline \multirow{5}{*}{$\begin{array}{l}\text { Ankle Max } \\
\text { Abs (W/kg) }\end{array}$} & A & $-0.36 \pm 0.32$ & $-0.41 \pm 0.37$ & $-0.41 \pm 0.31$ & $-0.45 \pm 0.31$ \\
\hline & UA & $-0.63 \pm 0.27$ & $-0.50 \pm 0.23$ & $-0.58 \pm 0.23$ & $-0.61 \pm 0.33$ \\
\hline & UA-A & $-0.26 \pm 0.23^{*}$ & $-0.19 \pm 0.19 *$ & $-0.17 \pm 0.19$ & $-0.16 \pm 0.28$ \\
\hline & $\Delta \mathbf{A}$ & - & $-0.01 \pm 0.09$ & $-0.05 \pm 0.10$ & $-0.08 \pm 0.13$ \\
\hline & $\Delta \mathbf{U A}$ & - & $0.15 \pm 0.12$ & $-0.08 \pm 0.23$ & $0.02 \pm 0.17$ \\
\hline \multirow{5}{*}{$\begin{array}{l}\text { Ankle total } \\
\text { +ve work } \\
(\mathrm{J} / \mathbf{k g})\end{array}$} & $\mathbf{A}$ & $4.95 \pm 4.54$ & $4.95 \pm 5.21$ & $4.81 \pm 4.86$ & $4.74 \pm 4.44$ \\
\hline & UA & $19.80 \pm 10.85$ & $18.56 \pm 12.54$ & $19.96 \pm 10.14$ & $20.62 \pm 13.25$ \\
\hline & UA-A & $14.85 \pm 7.12$ & $13.80 \pm 8.20$ & $15.15 \pm 6.31$ & $15.89 \pm 10.75$ \\
\hline & $\Delta \mathbf{A}$ & - & $-0.70 \pm 1.69$ & $-0.14 \pm 2.00$ & $-0.21 \pm 2.32$ \\
\hline & $\Delta \mathbf{U A}$ & - & $-2.01 \pm 2.43$ & $0.17 \pm 3.72$ & $0.83 \pm 8.07$ \\
\hline \multirow{5}{*}{$\begin{array}{c}\text { Ankle total } \\
\text {-ve work } \\
\text { (J/kg) }\end{array}$} & $\mathbf{A}$ & $-10.51 \pm 9.51$ & $-11.20 \pm 11.05$ & $-11.50 \pm 10.76$ & $-12.33 \pm 9.65$ \\
\hline & UA & $-15.17 \pm 6.96^{*}$ & $-11.84 \pm 4.37 *$ & $-14.40 \pm 5.63$ & $-15.86 \pm 8.40$ \\
\hline & UA-A & $-4.65 \pm 5.73 *$ & $-2.28 \pm 7.58^{*}$ & $-2.91 \pm 6.86$ & $-3.54 \pm 5.42$ \\
\hline & $\Delta \mathbf{A}$ & - & $0.61 \pm 2.92$ & $-0.98 \pm 3.77$ & $-1.81 \pm 2.39$ \\
\hline & $\Delta \mathbf{U A}$ & - & $4.89 \pm 3.14$ & $0.76 \pm 2.86$ & $-0.69 \pm 4.69$ \\
\hline \multirow{5}{*}{$\begin{array}{l}\text { Knee total } \\
\text { +ve work } \\
\quad(\mathrm{J} / \mathrm{kg})\end{array}$} & $\mathbf{A}$ & $4.45 \pm 1.90$ & $3.92 \pm 2.47$ & $4.81 \pm 1.77$ & $4.59 \pm 2.49$ \\
\hline & UA & $3.94 \pm 2.62$ & $3.25 \pm 3.34$ & $3.02 \pm 1.73$ & $2.68 \pm 1.86$ \\
\hline & UA-A & $-0.51 \pm 3.38$ & $-0.20 \pm 3.88$ & $-1.79 \pm 2.50$ & $-1.91 \pm 3.02$ \\
\hline & $\Delta \mathbf{A}$ & - & $0.08 \pm 0.78$ & $0.36 \pm 0.60$ & $0.14 \pm 1.76$ \\
\hline & $\Delta \mathbf{U A}$ & - & $0.42 \pm 1.77$ & $-0.93 \pm 1.25$ & $-1.26 \pm 2.66$ \\
\hline \multirow{5}{*}{$\begin{array}{c}\text { Knee total } \\
\text {-ve work } \\
(\mathrm{J} / \mathrm{kg})\end{array}$} & $\mathbf{A}$ & $-6.40 \pm 2.31$ & $-5.45 \pm 3.57$ & $-6.73 \pm 2.21$ & $-7.48 \pm 2.70$ \\
\hline & UA & $-11.19 \pm 6.41$ & $-9.91 \pm 7.62$ & $-9.83 \pm 4.68$ & $-9.22 \pm 4.45$ \\
\hline & UA-A & $-4.79 \pm 7.87$ & $-5.97 \pm 7.59$ & $-3.10 \pm 5.42$ & $-1.74 \pm 5.19$ \\
\hline & $\Delta \mathbf{A}$ & - & $0.33 \pm 0.60$ & $-0.32 \pm 1.44$ & $-1.08 \pm 1.95$ \\
\hline & $\Delta \mathbf{U A}$ & - & $1.14 \pm 5.55$ & $1.36 \pm 4.90$ & $1.97 \pm 6.37$ \\
\hline \multirow{5}{*}{$\begin{array}{l}\text { Hip total +ve } \\
\text { work }(J / k g)\end{array}$} & $\mathbf{A}$ & $8.37 \pm 6.64$ & $9.29 \pm 9.14$ & $8.87 \pm 6.10$ & $8.39 \pm 6.17$ \\
\hline & $\mathbf{U A}$ & $17.21 \pm 5.81$ & $12.92 \pm 3.35$ & $10.59 \pm 5.23$ & $17.71 \pm 6.51$ \\
\hline & UA-A & $8.84 \pm 8.13^{*}$ & $4.84 \pm 12.96$ & $1.72 \pm 8.10^{*}$ & $9.33 \pm 10.34$ \\
\hline & $\Delta \mathbf{A}$ & - & $0.05 \pm 3.54$ & $0.50 \pm 2.49$ & $0.01 \pm 4.44$ \\
\hline & $\Delta \mathbf{U A}$ & - & $-5.78 \pm 6.45$ & $-6.62 \pm 7.07$ & 0.5011 .55 \\
\hline \multirow{5}{*}{$\begin{array}{l}\text { Hip total -ve } \\
\text { work }(\mathrm{J} / \mathrm{kg})\end{array}$} & A & $-1.38 \pm 1.18$ & $-0.79 \pm 0.50$ & $-0.69 \pm 0.53$ & $-1.27 \pm 0.74$ \\
\hline & UA & $-2.03 \pm 1.33$ & $-2.26 \pm 1.63$ & $-2.05 \pm 1.82$ & $-2.30 \pm 2.35$ \\
\hline & UA-A & $-0.65 \pm 1.52$ & $-1.63 \pm 1.96$ & $-1.37 \pm 2.04$ & $-1.03 \pm 2.51$ \\
\hline & $\Delta \mathbf{A}$ & - & $0.72 \pm 1.00$ & $0.69 \pm 1.34$ & $0.11 \pm 1.59$ \\
\hline & $\Delta \mathbf{U A}$ & - & $-0.97 \pm 1.54$ & $-0.02 \pm 2.61$ & $-0.27 \pm 2.70$ \\
\hline
\end{tabular}

$\mathrm{A}=$ Affected side; $\mathrm{UA}=$ Unaffected side; $\mathrm{UA}-\mathrm{A}=$ Difference between unaffected and affected sides; $\Delta$ = Change scores relative to baseline; Max Gen = Maximum generation; Max Abs = Maximum absorption; Total +ve work $=$ Total positive work (total power generation); Total - ve work $=$ Total negative work (total power absorption); $\mathbf{T} 1=\mathrm{Two}$ weeks post-injection; $\mathrm{T} 2=$ Six weeks post-injection; $\mathrm{T} 3=$ Ten weeks post-injection $* \mathbf{p}<\mathbf{0 . 0 5}$ 

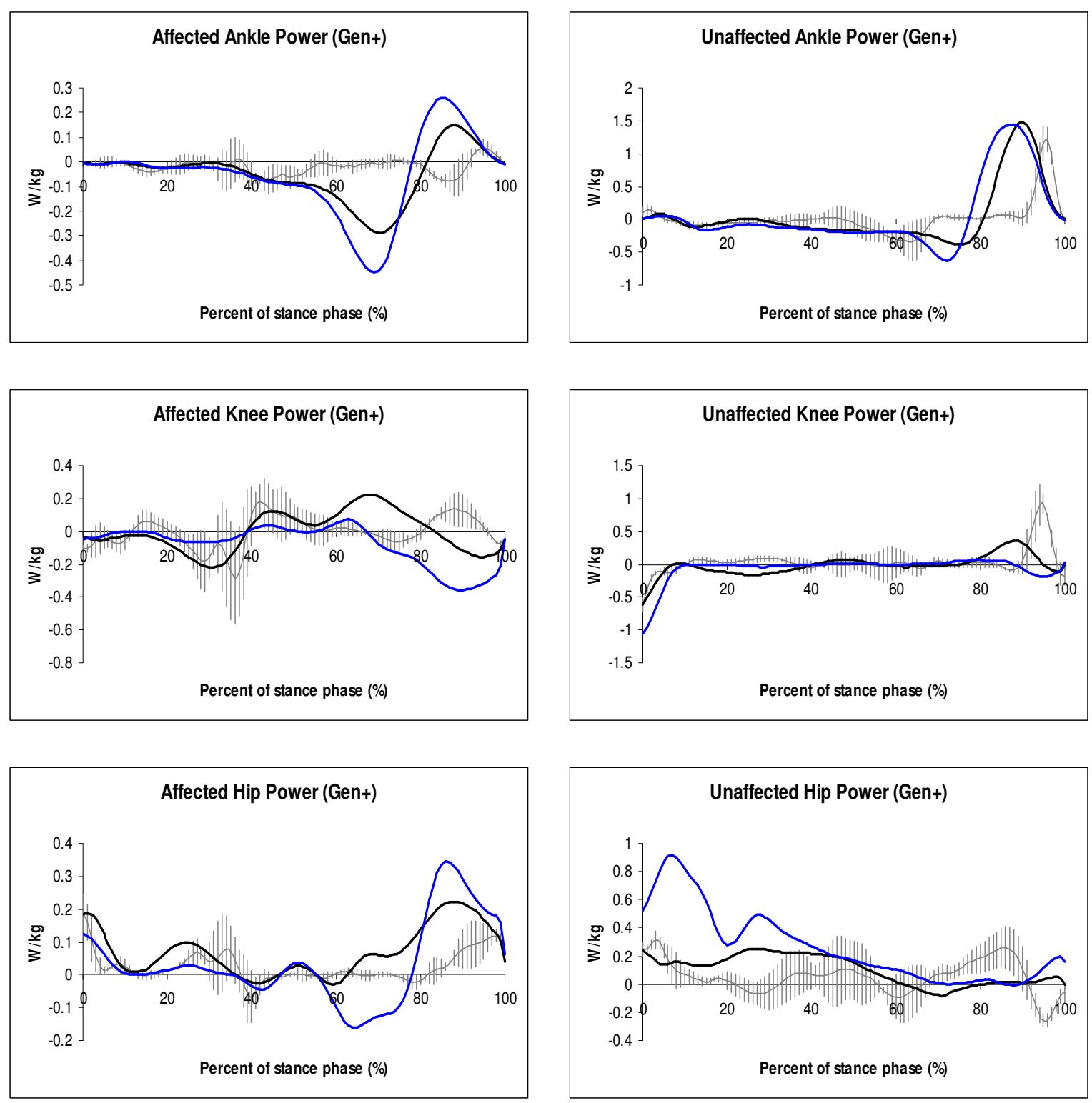

Figure 6a Powers (W/kg) at the ankle, knee and hip of a "responder" (subject 1) at Baseline (light grey), T1 (red), T2 (black) and T3 (blue). Shaded area reflects the standard deviation of the two baseline measures. Note the different scales on Y-axis for the affected and unaffected sides. Data at T1 were not available for subject 1. 

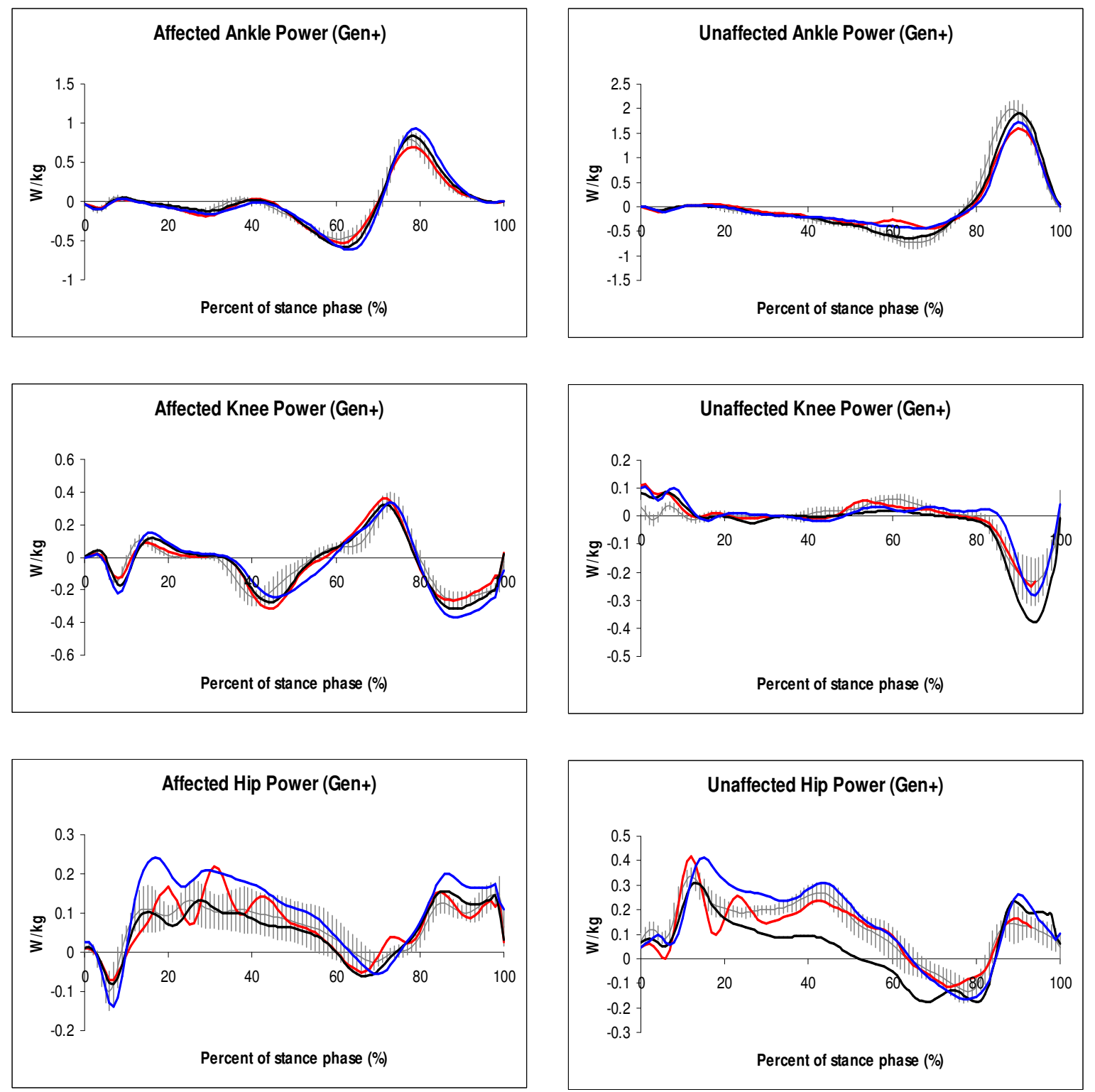

Figure 6b Powers (W/kg) at the ankle, knee and hip of a "non-responder" (subject 2) at Baseline (light grey), T1 (red), T2 (black) and T3 (blue). Shaded area reflects the standard deviation of the two baseline measures. Note the different scales on $\mathbf{Y}$-axis for the affected and unaffected sides. 
Table 8

Summary of individual clinical, kinematic and kinetic changes over time (T1 and T3)

\begin{tabular}{|c|c|c|c|c|c|c|c|}
\hline \multirow{2}{*}{ Subject } & \multirow{2}{*}{ Time } & \multirow{2}{*}{ Clinical } & \multirow{2}{*}{$\begin{array}{c}\text { Gait } \\
\text { speed }\end{array}$} & \multicolumn{2}{|c|}{ Ankle mobility } & \multirow{2}{*}{$\begin{array}{c}\text { Ankle } \\
\text { plantarflexor } \\
\text { work } \\
\text { Affected side }\end{array}$} & \multirow{2}{*}{$\begin{array}{c}\text { Positive hip } \\
\text { work } \\
\text { Affected } \\
\text { side }\end{array}$} \\
\hline & & & & $\begin{array}{c}\text { Max } \\
\text { DF }\end{array}$ & $\begin{array}{c}\text { Max } \\
\text { PF }\end{array}$ & & \\
\hline \multirow{2}{*}{1} & T1 & + & ++ & ++ & - & N/A & N/A \\
\hline & T3 & & ++ & ++ & ++ & ++ & ++ \\
\hline \multirow{2}{*}{2} & $\mathbf{T 1}$ & - & - & ++ & -- & - & + \\
\hline & $\mathbf{T 3}$ & & ++ & ++ & ++ & ++ & ++ \\
\hline \multirow{2}{*}{3} & $\mathbf{T 1}$ & + & - & ++ & + & -- & ++ \\
\hline & T3 & & - & ++ & - & -- & ++ \\
\hline \multirow{2}{*}{4} & T1 & - & + & -- & -- & -- & -- \\
\hline & T3 & & - & ++ & ++ & ++ & -- \\
\hline \multirow{2}{*}{5} & $\mathbf{T 1}$ & + & - & - & + & -- & - \\
\hline & $\mathbf{T 3}$ & & -- & + & ++ & -- & -- \\
\hline \multirow{2}{*}{6} & $\mathbf{T 1}$ & + & + & - & + & ++ & ++ \\
\hline & $\mathbf{T 3}$ & & - & ++ & ++ & - & ++ \\
\hline \multirow{2}{*}{7} & T1 & + & -- & -- & + & -- & -- \\
\hline & T3 & & - & -- & + & -- & ++ \\
\hline
\end{tabular}

'+' = Improvement (positive change); ' - '= No improvement (negative or no change); T1 = Two weeks post-injection; ' $++{ }^{\prime}=\geq 10 \%$ improvement over baseline; ' $--{ }^{\prime}=\leq 10 \%$ decline over baseline; T3 = Ten weeks post-injection; Max DF = Maximum dorsiflexion angle; $\mathrm{Max} \mathrm{PF}=$ Maximum plantarflexion angle; $\mathrm{N} / \mathrm{A}=$ Data not available 


\section{CHAPTER 5}

\section{DISCUSSION}

Currently there is a paucity of research describing the biomechanics of gait in association with BTX-A treatment in persons with stroke. As the primary objective of the current study, the kinematic and kinetic variables of gait have been described prior to and after BTX-A injection of the hypertonic plantarflexor muscles. A secondary objective of the study was to identify clinical characteristics of those individuals who present with biomechanical improvements post-injection.

The main findings of the current study were a general improvement in ankle kinematics in the group of subjects following BTX-A treatment. Greater dorsiflexion was observed during stance, and at initial contact the foot was approaching a neutral position compared to baseline. These changes, however, were not accompanied by any detectable alterations in gait kinetics nor changes in temporal distal parameters of gait posttreatment. Given the small sample size and the heterogeneity of the cohort it is conceivable that analyses of the pooled data may have masked important individual changes in gait patterns; for this reason individual data were evaluated in order to detect changes post-injection.

\section{Baseline (Pre-Treatment)}

Gait speed is a reflection of stroke severity and severity of impairment (Kramers de Quervain et al., 1996). Subjects in the current study walked an average of $0.44 \pm 0.24$ $\mathrm{m} / \mathrm{s}$ indicating moderate severity, with subjects 1 and 3 walking at a slower speed (0.12 to 
$0.24 \mathrm{~m} / \mathrm{s})$ and subjects 2,5 and 6 walking faster $(0.56$ to $0.74 \mathrm{~m} / \mathrm{s})$. The observed range of gait speeds is in accordance with other studies on stroke gait (Kim and Eng, 2004; Kramers de Quervain et al., 1996; Olney et al., 1991; Wall and Turnball, 1986) and clearly illustrates the extent to which these individuals' walking ability is compromised relative to similarly aged healthy subjects $(1.27 \mathrm{~m} / \mathrm{s}$ to $1.39 \mathrm{~m} / \mathrm{s}$ for men and women aged 50 to 70 ; Bohannon, 1997).

From a clinical standpoint, all subjects initially presented with hypertonicity of the ankle plantarflexors that limited ankle mobility. The increased tone was accompanied by abnormal movement patterns during gait including initial contact in excessive plantarflexion and limited plantarflexor push-off. Similar observations have been made by others (Boyd and Graham, 1997; Knutsson and Richards, 1979; Olney and Richards, 1996). Knutsson and Richards (1979) have described abnormal muscle activity during gait in persons with hemiparesis and classified individuals based on the nature of the abnormality recorded using electromyography, EMG (Type I: premature activation of calf musculature; Type II: extremely low activity of the calf muscles; Type III: coactivation of the plantar- and dorsiflexors). Type I and Type II muscle activation patterns were associated with knee hyperextension during stance and initial contact in plantarflexion rather than neutral (Knutsson and Richards, 1979). In this study, all subjects demonstrated an abnormal degree of ankle plantarflexion at initial contact along with excessive maximum knee extension on the affected side during stance at baseline compared with healthy individuals (Winter, 1991). While these patterns may be secondary to impaired activation of the plantarflexors as described as Type I and Type II above, in the absence of recording EMG it is not possible to attribute kinematic findings 
to neuromuscular sources with any certainty. Of note, however is that Olney and Richards (1996) have also linked hypertonic ankle plantarflexors with several kinematic abnormalities including knee hyperextension and exaggerated ankle plantarflexion during stance. Given the baseline biomechanical gait profiles of the subjects in this study, a treatment targeting the high tone plantarflexors should reasonably be expected to result in improved walking performance.

\section{Effects of BTX-A}

Gait analysis was performed at three time intervals post-injection two- (T1), six(T2) and ten (T3) weeks post-injection though discussion is limited to T1 in order to capture the peak treatment effect and T3 in order to assess the sustainability of the treatment once the paralytic effects of the drug have subsided. T2 measurements provided an interim evaluation which may have proved valuable had the time course revealed unexpected changes. As it was, any gait alterations were progressive with the T2 observations supplying little additional information.

\section{Clinical Measures}

The effect of BTX-A is to block muscle activation at the neuromuscular junction rendering the muscle less able to produce force (weakness). In a hypertonic muscle with elevated intrinsic stiffness as frequently occurs in chronic stroke (Rydahl and Brouwer, 2004) this would effectively reduce the resistance to passive stretch and allow the dorsiflexors to better exert their influence of active joint mobility. In subjects with hyperreflexia, BTX-A should limit the responsiveness to stretch, but 2 subjects (subject 2 and 
4) showed no clinical improvements in ankle mobility, reduced resistance to plantarflexor stretch (MAS) and the resolution of clonus. In the subjects who failed to exhibit any clinical sign of improvement following BTX-A it is possible that the injection site was suboptimal although with EMG guidance this seems unlikely. Perhaps it is the clinical measures themselves that lack the resolution to detect small magnitudes of change which may nonetheless yield functional gains.

\section{Temporal distal parameters}

From the reported research, some authors have noted significant improvement in gait velocity (2 to 12 weeks) after BTX-A treatment (Hesse et al., 1994; Johnson et al., 2004; Reiter et al., 1998) whereas, others found no change (Burbaud et al., 1996; Cioni et al., 2006). In the current study, overall the group of subjects did not walk any faster following treatment. Individually though, two subjects (subject 1 and 2) showed sustained improvements after 10 weeks. Subject 1 more than tripled his gait speed from baseline and subject 2 walked $12 \%$ faster. Interestingly, from a clinical standpoint, subject 1 was considered a "responder" since he showed gains in ankle mobility (active and passive) following BTX-A injection and also no longer exhibited clonus. In contrast, subject 2 showed no clinical improvement after treatment.

Other temporal distal parameters of gait, such as step length and stance time, have been shown to improve post BTX-A injection as well (Hesse et al., 1994; Reiter et al., 1998). In this study, subject 1 increased his step length, however no significant changes in step length or stance time on either the affected or unaffected side were seen for the group. This was not because the changes were masked by the variability within the 
sample since examination of the change scores also failed to identify any improvement. The discrepancy between the current findings and others (Hesse et al., 1994; Reiter et al., 1998) may be related to the use of assistive devices. In the current study subjects did not use canes or AFO's during testing, although 5 out of the 7 subjects in the study used assistive devices daily. Without them subjects may have walked more slowly and conservatively than they normally would with their cane. In Hesse et al.'s (1994) study subjects who routinely used assistive devices also used them during testing.

\section{Kinematics}

In accordance with the literature (Corry and Graham, 1998; Hesse et al., 1994; Sutherland et al., 1999) significant improvements in ankle kinematics were seen at ten weeks post-injection. When hypertonicity of the ankle plantarflexors substantially restricts passive movement, rotation of the ankle during stance is limited and contact is

often made on the forefoot instead of the heel (Perry et al., 1978). Following BTX-A treatment, subjects were able to achieve increased dorsiflexion range and the excursion in plantarflexion was reduced. It is likely that the increased compliance of the plantarflexor muscles allowed rotation of the leg over the foot during stance to occur therefore resulting in greater dorsiflexion (see also Sutherland et al., 1999). This effect was evident at 2 weeks but was significantly improved at T3 (ten weeks post-injection) suggesting that gains in muscle dispensability were sustained.

The ankle position at initial contact post-injection changed from plantarflexion at baseline toward a neutral position at T3 in all but one subject. This finding is consistent with the results of Hesse et al. (1994) and may reflect the enhanced ability of the 
dorsiflexor to mobilize the ankle joint without having to counter the hypertonic plantarflexors. Only subject 5 failed to exhibit any improvement at initial contact even though he was classified as a "responder" clinically. Interestingly, this subject was the only individual to reduce his gait speed (by $0.14 \mathrm{~m} / \mathrm{s}$ ) at $\mathrm{T} 3$ relative to pre-treatment. This may reflect a prolonged paresis affecting both plantar- and dorsiflexors such that the ability of the dorsiflexors to position the ankle optimally prior to initial contact remained compromised. Without measures of strength however, the legitimacy of this explanation cannot be confirmed.

Hesse et al. (1994) suggested that shift from initial forefoot contact to a more neutral position supports the body's progression through stance, and this reflects improved kinetics. Premature activity of the plantarflexors, with onset in terminal swing, is an important reason for inappropriate plantarflexion in early stance (Perry et al., 1978). Hesse et al. (1996) have shown a reduction in the activity of calf muscles in terminal swing and early stance following BTX-A treatment. Normalization of muscle activation patterns may account for the improved ankle position at initial contact in the current study though confirmation with EMG data is needed.

It is noteworthy that significant kinematic changes were not evident until T3 although changes appeared to be progressive from the early post-treatment. It is possible that following BTX-A treatment subjects needed time to adapt to the altered plantarflexor tone and sequelae in order to regain a sense of stability while walking. Considering that the magnitude of the neuromuscular blockade begins to diminish after about two weeks to twelve weeks, the synergist and antagonist muscles would necessarily be adapting in 
response to alterations in plantarflexor tone, with these adaptations stabilizing only after a greater amount of time.

Perhaps not unexpectedly, the angular kinematics associated with the hip and knee were stable for subjects over the study period with one exception. Subjects who showed improved position at initial contact also, on visual inspection showed less knee hyperextension in stance (e.g. Figure 5).

\section{Powers and work}

Power and work both lend insight into muscle performance. From a gait efficiency viewpoint the ability of the plantarflexors, hip extensors and hip flexors to generate power and perform work is particularly important as these muscles groups are major contributors (Olney et al., 1991) and enhance forward progression. Furthermore, in stroke, reduced plantarflexor power at push-off is generally compensated by increased hip flexor power generation to move the limb through swing (Nadeau et al., 1999; Olney et al., 1996). Therefore examining the work performed and the power generated by the ankle and hip muscles provides a means of evaluating the effectiveness of BTX-A treatment. Although no significant changes in power or work were seen for the group as a whole at T3, subjects 3, 6 and 7 performed less work by the plantarflexors and more work by the hip extensors and flexors 10 weeks after treatment. These results may be explained in terms of muscle weakness. Following stroke, hypertonicity occurs as only one part of the upper motor neuron syndrome. Muscle weakness is also a common consequence of hemispheric stroke and has been implicated as a factor underlying most limitations in physical task performance including gait deficits (Bohannon and Andrews, 
1990). As shown in children with cerebral palsy, spasticity and strength are not linked causally; spasticity does not cause muscle weakness (Rose and Engsberg, 2002). The difficulty is in distinguishing the effects of one over the other (hypertonicity and weakness) on physical performance since in many cases they coexist. Furthermore, distal muscles are generally more affected than proximal muscles (Brouwer and Ashby, 1991; Brouwer, 2000). It follows that decreasing plantarflexor tone with BTX-A would allow the full extent of plantarflexor weakness to manifest; thus in subjects 3,6 and 7 the hip extensors and flexors were required to generate sufficient power to compensate. For the hip extensors, that power increase is functionally important as it is associated with moving the trunk forward over the stance foot, therefore providing the hip flexors with a better mechanical advantage to perform the work of pulling off the limb in terminal stance (Teixeira-Salmela et al., 2001).

Others have also concluded that weakness of the ankle muscles is compensated by greater use of the hip flexors in terminal stance to pull the limb through swing and maintain gait speed (Nadeau et al., 1999). Strength training in conjunction with BTX-A treatment may have been optimally beneficial in these subjects in order to maximize benefits of tone reduction on mobility and develop plantarflexor strength. Krishnan (2006) has noted that although BTX-A relieves spasticity, it does not bring about motor recovery on its own. It can however, play a role as a "neuromotor relearning tool" (pg. 135) and improvement in function can be maximized when BTX-A is accompanied by activity based relearning.

Subject 5 did not show any changes in the ability of the ankle or hip muscle to produce work following treatment. This is consistent with the absence of any alterations 
in kinematic measures. In fact, the ability to generate power at the ankle and hip were somewhat less post-treatment than baseline. This likely accounts for the reduction in gait speed observed in this subject. Hesse et al., (1996) have also noted subjects who did not benefit from BTX-A treatment, and characterized them as displaying an EMG Type III pattern, in accordance with Knutsson and Richards (1979); that is a completely disrupted activation pattern of several or all limb muscles. In such instances, modifying the contractility of only one muscle group would be unlikely to result in any demonstrable gain if the other limb muscles showed abnormal recruitment and derecruitment patterns (Levin and Hui-Chan, 1994).

Subjects 1 and 2 increased the positive work performed by the ankle plantarflexors and the hip extensors/flexors post-injection and subject 4 increased the work output of the ankle plantarflexors only. Intuitively, the reduction in plantarflexor tone by blocking the development of active tension should result in a reduction in pushoff power. However, by T3 the effects of BTX-A have subsided so in cases where a reduction in muscle stiffness is sustained allowing for increased passive dorsiflexion as the leg rotates over the foot, the plantarflexors are mechanically advantaged to produce greater push-off (Boyd et al., 2000). In addition, the normalized ankle kinematics could delay activation of the stretch reflex until terminal stance thereby contributing to the power generation at push-off (Boyd et al., 2000; Hesse et al., 1996). The combined benefit of increased work capacity of the plantarflexors and hip extensors/flexors likely contributed to the increased gait speed observed in subjects 1 and 2 . 


\section{Clinical and biomechanical effects}

It is important to identify the clinical characteristics that differentiate those subjects who show biomechanical improvements following BTX-A from those who do not in order to better select suitable candidates. Based on clinical measures of impairment that have been shown to be responsive to the effects of BTX-A post-stroke, the subjects in this study were classified as either "responders" or "non-responders" to the treatment. If ankle mobility improved and spasticity or resistance to passive stretch decreased, then the individual was classed as a "responder". Subjects 2 and 4 were considered "nonresponders" yet they did not appear different from others at baseline. Furthermore, in contrast with the "non-responder" classification, subject 2 showed both kinematic and kinetic improvements post-treatment. Subject 4 demonstrated only modest improvements in kinematic and kinetic measures at the ankle and hip. In contrast, subject 5, who was clinically considered a "responder" did not improve his ankle kinematics or ability to generate power or perform work. Indeed this subject reduced his gait speed following treatment. At baseline this subject had above average active ROM and below average passive ROM, but not to an extent that indicated a fixed contracture.

From the clinical measurements recorded in this study there are no prognostic indicators of who would or would not benefit from BTX-A treatment. Perhaps testing the strength of ankle musculature would provide a means of selecting those most likely to respond to treatment as those with weaker plantar- and dorsiflexors may be less able to generate power even if tone is reduced (Teixeira-Salmela et al., 2001).

In summary, some subjects showed marked improvements in gait performance however there was not a clear clinical profile identifying those subjects who would 
respond best or not respond at all. A more comprehensive clinical profile may be required in order to better target BTX-A treatments. 


\section{CHAPTER 6}

\section{CONCLUSIONS AND RECOMMENDATIONS}

\section{Conclusion}

The present study has demonstrated that BTX-A treatment of the hypertonic ankle plantarflexors improved ankle kinematics, and the ability of the ankle and hip muscles to perform work to improve gait speed and efficiency in select subjects.

The major findings of the current study are summarized below.

- Significant gains in ankle dorsiflexion during stance for the stroke group as a whole

- Major kinematic changes were observed at T3 (ten weeks post-injection) relative to baseline including improved ankle position at initial contact in the group as a whole.

- In select subjects, marked increases in gait speed in association with increased dorsiflexion, and decreased plantarflexion during stance, more neutral ankle angle at initial contact, and increases in plantarflexor and hip extensor and flexor positive work performed during stance were observed.

- There was an absence of a clinical profile identifying those subjects who benefit most from BTX-A treatment in terms of walking performance.

- Subjects who were identified as "responders" or "non-responders" based on clinical measures were not necessarily those who showed (or did not show) biomechanical changes in gait suggesting a dissociation between the these evaluation methods..

\section{Limitations}

The following were limitations of the study: 
- The small sample size $(n=7)$ paired with subject heterogeneity limited the power to detect changes over time following treatment. The generalizability of the findings to the chronic stroke population is also limited.

- The lack of EMG data to accompany the kinematic and kinetic data made it difficulty to determine the mechanisms accounting for the power and work profiles observed pre and post BTX-A treatment. EMG would have been valuable in exploring the muscle contributions associated with observed changes at the ankle in particular.

- The injection sites and dosage of BTX-A were not standardized for all subjects. As this was an open label clinical trial it was important that the treatment was delivered in a manner consistent with clinical practice. A single physician was responsible for all clinical decision making with respect to BTX-A treatment.

\section{Recommendations for future study}

It is recommended that:

- A larger sample of people with plantarflexor hypertonicity due to stroke be recruited for testing

- EMG data be incorporated into the gait analysis

- Effects of BTX-A be investigated over a longer period of time. Significant kinematic effects were first seen at 10 weeks post-injection therefore a longer time period may reveal further trends

- The effects of BTX-A in conjunction with strength training be explored in addition to BTX-A in isolation 
- More comprehensive clinical evaluation be introduced (e.g. measures of strength) in order to improve prognosis of who will most likely benefit from treatment 


\section{REFERENCES}

1. Andriacchi TP, Ogle JA, Galante JO. Walking speed as basis for normal and abnormal gait measurements. Journal of Biomechanics 1977; 10: 261-268.

2. Bayram S, Sivrioglu K, Karli N, Ozcan O. Low dose botulinum toxin with short term electrical stimulation in poststroke spastic drop foot: a preliminary study. American Journal of Physical Medicine and Rehabilitation 2006; 85: 75-81.

3. Bergfeldt U, Borg K, Kullander K, Julin P. Focal spasticity therapy with botulinum toxin: effects on function, activities of daily living and pain in 100 adult patients. Journal of Rehabilitative Medicine 2006; 38: 166-171.

4. Blasi J, Chapman ER, Link E, Binz T, Yamasaki S, De Camilli P, Sudhof TC, Niemann H, Jahn R. Botulinum neurotoxin A selectively cleaves the synaptic protein SNAP-25. Nature 1993; 365: 160-163.

5. Bohannon RW, Smith MB. Interrater reliability of a modified Ashworth Scale for muscle spasticity. Journal of the American Physical Therapy Association 1987; 67: 206-207.

6. Bohannon RW, Andrews AW, Smith MB. Rehabilitation goals of patients with hemiplegia. International Journal of Rehabilitation Research 1988; 11: 181-182.

7. Bohannon RW, Andrews A. Correlation of knee extension torque and spasticity with gait speed in patients in stroke. Archives of Physical Medicine and Rehabilitation 1990; 71: 330-333.

8. Bohannon RW, Horton MG, Wikholm Jb. Importance of 4 variables of walking to patients with stroke. International Journal of Rehabilitation Research 1991; 14: 246-250.

9. Bohannon RW. Comfortable and maximum walking speed of adults aged 20-79 years: reference values and determinants. Age and Ageing 1997; 26: 15-19.

10. Booth CM, Cortina-Borja MJ, Theologis TN. Collagen accumulation in muscles of children with cerebral palsy and correlation with severity of spasticity. Developmental Medicine and Child Neurology 2001; 43: 314-320.

11. Bottos M, Benedetti MG, Salucci P, Gasparroni V, Giannini S. Botulinum toxin with and without casting in ambulant children with spastic diplegia: a clinical and functional assessment. Developmental Medicine and Child Neurology 2003; 45: 758-762. 
12. Boyd R, Graham HK. Botulinum toxin A in the management of children with cerebral palsy: indications and outcome. European Journal of Neurology 1997; 4: S15-S22.

13. Boyd R, Pliatsios V, Starr R, Wolfe R, Graham HK. Biomechanical transformation of the gastroc-soleus muscle with botulinum toxin A in children with cerebral palsy. Developmental Medicine and Child Neurology 2000; 42: 32-41.

14. Brouwer B. Strength training in long term stroke survivors. BioMechanics 2000; 7:61-70.

15. Brouwer B, Ashby P. Altered corticospinal projections to lower limb motoneurons in subjects with cerebral palsy. Brain 1991; 114: 1395-1407

16. Brouwer B, Wheeldon RK, Stradiotto-Parker N. Reflex excitability and isometric force production in cerebral palsy: the effect of serial casting. Developmental Medicine and Child Neurology 1998; 40: 168-175.

17. Burbaud P, Wiart L, Dubos JL, Gaujard E, Debelleix X, Joseph PA, Mazaux JM, Bioulac B, Barat M, Lagueny A. A randomized, double blind, placebo controlled trial of botulinum toxin in the treatment of spastic foot in hemiparetic patients. Journal of Neurology, Neurosurgery and Psychiatry 1996; 61: 265-269.

18. Burgen ASV, Dickens F, Zatman LJ. The action of botulinum toxin on the neuromuscular junction. Journal of Physiology 1949; 109: 10-24.

19. Chen G, Patten C, Kothari DH, Zajac FE. Gait differences between individuals with post-stroke hemiparesis and non-disabled controls at matched speeds. Gait and Posture 2005; 22: 51-56.

20.Cioni M, Esquenazi A, Hirai B. Effects of botulinum toxin A on gait velocity, step length and base of support of patients with dynamic equinovarus foot. American Journal of Physical Medicine and Rehabilitation 2006; 85: 600-606.

21. Corry IS, Cosgrove AP, Duffy CM, McNill A, Taylor TC, Graham HK. Botulinum toxin A compared with stretching casts in the treatment of spastic equines: a randomized prospective trial. Journal of Pediatric Orthopaedics 1998; 18: 304-311.

22. Cosgrove AP, Corry IS, Graham HK. Botulinum toxin A in the management of the lower limb in cerebral palsy. Developmental Medicine and Child Neurology 1994; 36: 386-396.

23. Davis EC, Johnson GR. The use of botulinum toxin in spasticity. In: Upper motor neurone syndrome and spasticity: clinical management and neurophysiology. Cambridge, United Kingdom: Cambridge University Press. 2001.

24.Dengler R, Neyer U, Wohlfarth K, Bettig U, Janzik HH. Local botulinum toxin in the treatment of spastic drop foot. Journal of Neurology 1992; 239: 375-378. 
25. Dietz V, Trippel M, Berger W. Reflex activity and muscle tone during elbow movements in patients with spastic paresis. Annals of Neurology 1991; 30: 767779.

26. Dunne JW, Heye N, Dunne SL. Treatment of chronic limb spasticity with botulinum toxin A. Journal of Neurology, Neurosurgery and Pyschiatry 1995; 58: 232-235.

27.Esquenazi A. Improvements in healthcare and cost benefits associated with botulinum toxin treatment of spasticity and muscle overactivity. European Journal of Neurology 2006; 13: 27-34.

28. Fock J, Galea MP, Stillman BC, Rawicki B, Clark M. Functional outcome following Botulinum toxin $\mathrm{A}$ injection to reduce spastic equinus in adults with traumatic brain injury. Brain Injury 2004; 18: 57-63.

29. Francisco GE, Boake C, Vaughn A. Botulinum toxin in upper limb spasticity after aquired brain injury: a randomized trial comparing dilution techniques. American Journal of Physical Medicine and Rehabilitation 2002; 81: 355-363.

30. Galli M, Crivelline M, Santambrogio GC, Fazzi E, Motta F. Short-term effects of "botulinum toxin A" as treatment for children with cerebral palsy: kinematic and kinetic aspects at the ankle joint. Functional Neurology 2001; 16: 317-323.

31. Goldie PA, Matyas TA, Evans OM. Gait after stroke: initial deficit and changes in temporal patterns for each gait phase. Archives of Physical Medicine and Rehabilitation 2001; 82: 1057-1065.

32. Gordon AM, Huxley AF, Julian FJ. The variation in isometric tension with sarcomere length in vertebrate muscle fibres. Journal of Physiology 1966; 184: 170-192.

33. Gracies JM, Marosszeky JE, Renton R, Sandanam J, Gandevia SC, Burke D. Short term effects of dynamic Lycra splints on upper limb in hemiplegic patients. Archives of Physical Medicine and Rehabilitation 2000; 81: 1547-1555.

34. Gunderson CB. The effects of botulinum toxin on the synthesis, storage and release of acetylcholine. Progress in Neurobiology 1980; 14: 99-119.

35. Hamill J, Selbie WS. Three-dimensional kinetics. In: Robertson DGE, Caldwell GE, Hamill J, Kamen G, Whittlesey SN (ed). Research methods in biomechanics. Windsor: Human Kinetics 2004; 145-160.

36. Heart and Stroke Foundation of Canada. Just the Facts: 2002/2003 Edition. 2002

37. Hesse S, Lucke D, Malezic M, Bertelt C, Friedrich H, Gregoric M, Mauritz KH. Botulinum toxin treatment for lower limb extensor spasticity in chronic 
hemiparetic patients. Journal of Neurology, Neurosurgery, and Psychiatry 1994; 57: 1321-1324.

38. Hesse S, Krajnik J, Luecke D, Jahnke MT, Gregoric M, Mauritz KH. Ankle muscle activity before and after botulinum toxin therapy for lower limb extensor spasticity in chronic hemiparetic patients. Stroke 1996; 27: 455-460.

39. Hoppenfeld S. Physical examination of the spine and extremities. Prentice-Hall: Norwalk, Connecticut. 1976.

40.Hsu AL, Tang PF, Jan MH. Analysis of impairments influencing gait velocity and asymmetry of hemiplegic patients after mild to moderate stroke. Archives of Physical Medicine and Rehabilitation 2003; 84: 1185-1193.

41.http://www.wemove.org/spa/

42. Huitema RB, Hof AL, Mulder T, Brouwer WH, Dekker R, Postema K. Functional recovery of gait and joint kinematics after right hemispheric stroke. Archives of Physical Medicine and Rehabilitation 2004; 85: 1982-1988.

43.Johnson CA, Burridge JH, Strike PW, Wood DE, Swain ID. The effect of combined use of botulinum toxin type A and functional electric stimulation in the treatment of spastic drop foot after stroke: a preliminary investigation. Archives of Physical Medicine and Rehabilitation 2004; 85: 902-909

44.Jorgensen HS, Nakayama H, Raaschou HO, Olsen TS. Recovery of walking function in stroke patients: the Copenhagen Stroke Study. Archives of Physical Medicine and Rehabilitation 1995; 76: 27-32.

45.Katz RT, Rymer WZ. Spastic hypertonia: mechanisms and measurement. Archives of Physical Medicine and Rehabilitation 1989; 70: 144-155.

46. Kerrigan CD, Frates EP, Rogan S, Riley PO. Spastic paretic stiff-legged gait. American Journal of Physical Medicine and Rehabilitation 1999; 4: 354-360.

47. Kim CM, Eng JJ. The relationship of lower extremity muscle torque to locomotor performance in people with stroke. Physical Therapy 2003; 83: 49-57.

48. Kim CM, Eng JJ. Magnitude and pattern of 3D kinematic and kinetic gait profiles in persons with stroke: relationship to walking speed. Gait and Posture 2004; 20: 140-146.

49. Koman LA, Mooney JF, Smith BP, Walker F, Leon JM. Botulinum toxin type A neuromuscular blockade in the treatment of lower extremity spasticity in cerebral palsy: a randomized, double-blind, placebo-controlled trial. Journal of Pediatric Orthopaedics 2000; 20: 108-115. 
50. Knutsson E, Richards C. Different types of disturbed motor control in gait of hemiparetic patients. Brain 1979; 102: 405-430.

51. Kramers de Quervain IA, Simon SR, Leurgans S, Pease WS, McAllister D. Gait pattern in the early recovery period after stroke. Journal of Bone and Joint Surgery 1996; 78: 1506-1514.

52. Krishan RV. Relearning toward motor recovery in stroke, spinal cord injury, and cerebral palsy: a cognitive neural systems perspective. International Journal of Neuroscience 2006; 116: 127-140.

53.Lamontagne A, Malouin F, Richards CL, Dumas F. Mechanisms of disturbed motor control in ankle weakness during gait after stroke. Gait and Posture 2002; 15: 244-255.

54.Lance JW, McLeod JG. A physiological approach to clinical neurology: $3^{\text {rd }}$ Edition. London: Butterworths. 1981.

55.Lee WA, Boughton A, Rymer WZ. Absence of stretch reflex gain enhancement in voluntarily activated spastic muscle. Experimental Neurology 1987; 98: 317-335.

56.Lehman JF, Condon SM, Price R, deLateur BJ. Gait abnormalities in hemiplegia: their correction by ankle-foot orthoses. Archives of Physical Medicine and Rehabilitation 1987; 68: 763-771.

57.Levin MF, Hui-Chan CW. Ankle spasticity is inversely correlated with antagonist voluntary contraction in hemiparetic subjects. Electromyography and Clinical Neurophysiology 1994; 34: 415-425.

58. Lin PY, Yang YR, Cheng SJ, Wang RY. The relation between ankle impairments and gait velocity and symmetry in people with stroke. Archives of Physical Medicine and Rehabilitation 2006; 87: 562-568.

59. Macko RF, DeSouza CA, Tretter LD, Silver KH, Smith GV, Anderson PA, Tomoyasu N, Gorman P, Dengel DR. Treadmill aerobic exercise training reduces the energy expenditure and cardiovascular demands of hemiparetic gait in chronic stroke patients. A preliminary report. Stroke 1997; 28: 326-330.

60. Mancini F, Sandrini G, Moglia A, Nappi G, Pacchetti C. A randomized, doubleblind, dose-ranging study to evaluate efficacy and safety of three doses of botulinum toxin type A (Botox) for the treatment of spastic foot. Neurological Science $2005 ; 26: 26-31$.

61. Mayo NE, Wood-Dauphinee S, Ahmed S, Gordon C, Higgins J, McEwen S, Salbach N. Disablement following stroke. Disability and Rehabilitation 1999; 21: 258-268. 
62. Mizrahi J, Susak Z, Heller L, Najenson T. Variation of time-distance parameters of the stride as related to clinical gait improvement in hemiplegics. Scandinavian Journal of Rehabilitation Medicine 1982; 14: 133-140.

63. Nadeau S, Arsenault AB, Gravel D, Bourbonnais D. Analysis of the clinical factors determining natural and maximal gait speeds in adults with a stroke. American Journal of Physical Medicine and Rehabilitation 1999; 78: 123-130.

64. Nadeau S, Gravel D, Arsenault B, Bourbonnais D. Plantarflexor weakness as a limiting factor of gait speed in stroke subjects and the compensating role of hip flexors. Clinical Biomechanics 1999; 14:125-135.

65. Naumann M, Albanese A, Heinen F, Molenaers G, Relja M. Safety and efficacy of botulinum toxin type A following long-term use. European Journal of Neurology 2006; 13: 35-40.

66. Niemi ML, Laaksonen R, Kotila M, Waltimo O. Quality of life 4 years after stroke. Stroke 1988; 19: 1101-1107.

67. Nolte, J. The human brain: an introduction to its functional anatomy: $5^{\text {th }}$ edition. St. Louis: Mosby Inc. 2002.

68. O'Dwyer NJ, Ada L, Neilson PD. Spasticity and muscle contracture following stroke. Brain 1996; 119: 17378-1749.

69. Olney SJ, Monga TN, Costigan PA. Mechanical energy of walking of stroke patients. Archives of Physical Medicine and Rehabilitation 1986; 67: 92-98.

70. Olney SJ, Jackson VG, George SR. Gait re-education guidelines for stroke paitents with hemiplegia using mechanical energy and power analyses. Physiotherapy Canada 1988; 40: 242-248.

71. Olney SJ, Griffin MP, Monga TN, McBride I. Work and power in gait of stroke patients. Archives of Physical Medicine and Rehabilitation 1991;72: 309-314.

72. Olney SJ, Griffin MP, McBride ID. Temporal, kinematic and kinetic variables related to gait speed in subjects with hemiplegia: a regression approach. Physical Therapy 1994; 74: 872-885.

73. Olney SJ, Richards C. Hemiparetic gait following stroke. Part I: Characteristics. Gait and Posture 1996; 4; 136-148.

74. On AY, Kirazli Y, Kismali B, Aksit R. Mechanisms of action of phenol block and botulinus toxin type A in relieving spasticity: electrophysiologic investigation and follow-up. American Journal of Physical Medicine and Rehabilitation 1999; 78 : 344-349. 
75. O'Sullivan SB, Schmitz TJ. Physical rehabilitation: assessment and treatment: $3^{\text {rd }}$ edition. Philadelphia: F.A. Davis Company. 1994.

76. Papadonikolakis AS, Vekris MD, Korompilias AV, Kostas JP, Ristanis SE, Soucacos PN. Botulinum A toxin for treatment of lower limb spasticity in cerebral palsy. Acta Orthopaedica Scandinavica 2003; 74: 749-755.

77.Parvataneni K, Olney SJ, Brouwer B. Changes in muscle group work associated with changes in gait speed of persons with stroke. Clinical Biomechanics 2007; 22: $813-820$.

78. Pearce LB, First ER, MacCallum RD, Gupta A. Pharmocologic characterization of Botulinum toxin for basic science and medicine. Toxixon 1997; 35: 1373-1412.

79.Peat M, Dubo HIC, Winter DA, Quanbury AO, Steinke T, Grahame R. Electromyographic temporal analysis of gait: hemiplegic locomotion. Archives of Physical Medicine and Rehabilitation 1976; 57: 421-425.

80.Perry J, Waters RL, Perrin T. Electromyographic analysis of equinovarus following stroke. Clinical Orthopaedics 1978; 131: 47-53.

81.Perry J, Garret M, Gronley JK, Mulroy SJ. Classification of walking handicap in the stroke population. Stroke 1995; 26: 982-989.

82. Pierson SH, Katz DI, Tarsy D. Botulinum toxin A in the treatment of spasticity: functional implications and patient selection. Archives of Physical Medicine and Rehabilitation 1996; 77: 717-721.

83. Potter JM, Evans Al, Duncan G. Gait speed and activities of daily living function in geriatric patients. Archives of Physical Medicine and Rehabilitation 1995; 76: 997-999.

84. Reiter F, Danni M, Lagalla G, Ceravolo G, Provinciali L. Low-dose botulinum toxin with ankle taping for the treatment of spastic equinovarus foot after stroke. Archives of Physical Medicine and Rehabilitation 1998; 79: 532-535.

85. Richards CL, Malouin F, Dumas F, Tardif D. Gait velocity as an outcome measure of locomotor recovery after stroke. In: Craik, RL, Oatis CA (Eds), Gait analysis. Mosby, Toronto. 1995.

86. Rosales RL, Arimura K, Takenaga S, Osame M. Extrafusal and intrafusal muscle effects in experimental botulinum toxin A injection. Muscle and Nerve 1996; 19: 488-496.

87. Rose SA, Engsberg JR. Relation between spasticity and strength in individuals with spastic diplegic cerebral palsy. Developmental Medicine and Child Neurology 2002; 44: 148-157. 
88. Roth EJ, Merbitz C, Mroczek K, Dugan SA, Suh WW. Hemiplegic gait. Relationships between walking speed and other temporal parameters. American Journal of Physical Medicine and Rehabilitation 1997; 76: 128-133.

89. Rousseaux M, Compere S, Launay MJ, Kozlowski O. Variability and predictability of functional efficacy of botulinum toxin injection in leg spastic muscles. Journal of the Neurological Sciences 2005; 232: 51-57.

90. Rydahl SJ, Brouwer BJ. Ankle stiffness and tissue compliance in stroke survivors: a validation of myotonometer measurements. Archives of Physical Medicine and Rehabilitation 2004; 85: 1631-1637.

91. Sarioglu B, Serdaroglu G, Tutuncuoglu S, Ozer EA. The use of botulinum toxin type A treatment in children with spasticity. Pediatric Neurology 2003; 29: 299301.

92. Sinkjaer T, Magnussen I. Passive, intrinsic, and reflex-mediated stiffness in the ankle extensors of hemiparetic patients. Brain 1994; 117: 355-363.

93. Suputtitada A, Suwanwela NC. The lowest effective dose of botulinum A toxin in adult patients with upper limb spasticity. Disability and Rehabilitation 2005; 18 : 176-184.

94. Sutherland DH, Kaufman KR, Wyatt MP, Chambers HG, Mubarak SJ. Doubleblind study of botulinum A toxin injections into the gastrocnemius muscle in patients with cerebral palsy. Gait and Posture 1999; 10: 1-9.

95. Suzuki S, Ohyama N, Yamada K, Kanamori M. The relationship between fear of falling, activities of daily living and quality of life among elderly individuals. Nursing and Health Science 2002; 4: 155-161.

96. Tardieu C, Huet de la Tour E, Bret MD, Tardieu G. Muscle hypoextensibility in children with cerebral palsy: I. Clinical and experimental observations. Archives of Physical Medicine and Rehabilitation 1982; 63: 97-102

97. Teixeira-Salmela LF, Nadeau S, McBride I, Olney SJ. Effects of muscle strengthening and physical conditioning training on temporal, kinematic and kinetic variables during gait in chronic stroke survivors. Journal of Rehabilitation Medicine 2001; 33: 53-60.

98. Thilmann AF, Fellows SJ, Garms E. The mechanism of spastic muscle hypertonus: variation in reflex gain over the time course of spasticity. Brain 1991; 114: 233244.

99. Turkel CC, Bowen B, Liu J, Brin MF. Pooled analysis of the safety of botulinum toxin type A in the treatment of poststroke spasticity. Archives of Physical Medicine and Rehabilitation 2006; 87: 786-792. 
100.Wade DT, Wood VA, Heller A, Maggs J, Hewer RL. Walking after stroke. Scandanavian Journal of Rehabilitation Medicine 1987; 19: 25-30.

101.Wall JC, Turnball GI. Gait asymmetries in residual hemiplegia. Archives of Physical Medicine and Rehabilitation 1986; 67: 550-553.

102.Watkins CL, Leathley MJ, Gregson JM, Smith TL, Sharma AK. Prevalence of spasticity post stroke. Clinical Rehabilitation 2002; 16: 515-522.

103.Whittle MW. Gait Analysis an introduction: second edition. Jordan Hill, Oxford: Butterworth-Heinemann. 1996.

104.Williams PE, Goldspink GE. Changes in sarcomere length and physiological properties in the immobilized muscle. Journal of Anatomy 1978; 127: 459-468.

105.Winter DA. Biomechanical motor patterns in normal walking. Journal of Motor Behaviour 1983; 15: 302-330.

106.Winter DA. Kinematic and kinetic patterns of human gait: variability and compensating effects. Human Movement Science 1984; 3: 51-76.

107.Winter DA. The biomechanics and motor control of human gait. Waterloo, Canada: University of Waterloo Press. 1987.

108. Winter DA. The biomechanics and motor control of human gait: normal, elderly and pathological. Waterloo, Canada: University of Waterloo Press. 1991.

109.Winter DA, Eng P. Kinetics: our window into the goals and strategies of the central nervous system. Behavioural Brain Research 1995; 67: 111-120.

110.Winter DA. The biomechanics and motor control of human gait: third edition. Hoboken, New Jersey: John Wiley \& Sons, Inc. 2005.

111. Zurcher AW, Molenaers G, Desloovere K, Fabry G. Kinematic and kinetic evaluation of the ankle after intramuscular injection of botulinum toxin A in children with cerebral palsy. Acta Orthopaedica Belgica 2001; 67: 475-479. 


\section{Appendix A}

\section{Modified Ashworth Scale}

$0 \quad$ No increase in muscle tone

1 Slight increase in muscle tone, manifested by catch and release or minimal resistance at the end of the motion when the affected part(s) is moved in flexion or extension

1+ Slight increase in muscle tone, manifested by a catch, followed by minimal resistance throughout the remainder (less than half) of the range of movement (ROM)

2 More marked increase in muscle tone through most of the ROM, but affected part(s) easily moved

3 Considerable increase in muscle tone, passive movement difficult

$4 \quad$ Affected part(s) rigid in flexion or extension

(Bohannon and Smith, 1987) 


\section{Appendix B}

\section{Consent form}

TITLE OF PROJECT: The Effects of Botulinum Toxin A (Btx-A) on

Plantarflexor Spasticity, Stiffness and Gait in Chronic Stroke

CO-INVESTIGATORS: Dr. Brenda Brouwer, School of Rehabilitation Therapy, Dr. Stephen Bagg, Providence Continuing Care Centre, St. Mary's of the Lake Hospital

\section{BACKGROUND INFORMATION}

You are being invited to participate in a research study because you have been identified as a candidate for Botulinum Toxin A (Btx-A) treatment for your stiff calf muscles (plantarflexors). Btx-A is a drug used to treat spasticity or excessive muscle stiffness and in some patients has been associated with improvements in ankle movement and reduction of pain. This study will evaluate the extent to which the appropriate dosage of Btx-A is associated with changes in muscle tone, walking ability, endurance and activity level. We are also interested in your opinion of the treatment.

One of the investigators will read through this consent form with you, describe the study procedures in detail and answer any questions you may have. Funding for the project is provided by an Unrestricted Education Research Fund from Allergan.

\section{DETAILS OF THE STUDY}

The purpose of this study is to determine the effectiveness of Btx-A treatment of the stiff calf muscles in people who have had a stroke. We will examine whether Btx-A treatment leads to changes in muscle tone, the ability to generate power from the calf muscles in isolation and during walking and if physical endurance and activity levels change after treatment. The information we obtain will help us to understand why some patients do better than others.

You are being considered for the study because you have had a stroke over 6 months ago, you are able to walk a distance of at least 10 metres (with or without a cane or orthotic device) and you have been seen by Dr. Bagg in the Stroke Out-Patient Clinic at St. Mary's of the Lake Hospital and he has identified you as someone who might benefit from receiving Btx-A treatment.

\section{Description of visits and tests to be performed as part of the study}

The study will require a total of 5 visits to the Motor Performance Laboratory in the School of Rehabilitation Therapy over a total period of twelve weeks. The first two visits will be scheduled two weeks apart and will provide baseline information relating to all 
outcome measures described below. After your second visit (week 2) you will receive the Btx-A treatment at St. Mary's of the Lake Hospital. At week 4 you will be asked to return for your third visit. Visits 4 and 5 will take place at four week intervals; that is week 8 and week 12.

During each visit the following measurements will be obtained.

1) We will measure muscle stiffness by moving your ankle and feeling the resistance to movement and measuring how much flexion and extension movement there is. In addition we will push a metal probe against the skin overlying your calf muscles while you are relaxed and also while you contract your muscles as hard as you can (three repetitions for each condition). The probe measures how much tissue displacement occurred.

2) We will measure how strong your ankle muscles are by having you sit on a comfortable chair with your foot attached to a footplate which in turn is attached to instruments that measure force output. You will be asked to push and pull as hard and as fast as you can by alternately pointing your toes and bringing them up toward your knee. The stiffness and strength measures will be obtained from both legs.

3) We will measure walking performance by placing a number of light emitting diodes (markers) on your skin at various landmarks on your left and right legs, hips and shoulders. This allows us to track the movement of your limbs as you walk on a walkway. You will be asked to walk at your comfortable speed along the 8 metre walkway several times while cameras detect the position of the markers. Rest breaks will be provided as needed.

4) We will measure your physical activity level by having you complete a questionnaire that asks about physical activities that you are doing or that you have stopped doing.

During visits 3-5 only, you will be asked about your satisfaction with the Btx-A treatment.

The total time required for each testing session is approximately 2.5 hours.

In addition to the information obtained through the testing described above, we will also require details about your stroke such as the type of stroke, structures affected by the stroke, previous stroke history and when your last stroke occurred. This information will be obtained from your medical chart by Dr. Bagg.

\section{Risks/Side-Effects:}

We have been conducting all the tests described above in the laboratory with many different patient groups (including people who have had strokes) and also in control subjects who have no physical impairments. There are no associated risks with the testing though occasionally after maximally contracting the muscles people report some activityrelated soreness within 48 hours after the testing. This is usually avoided by having you warm-up before testing and having you stretch and cool down afterwards. You may feel somewhat fatigued after testing depending on how active you normally are. This should not be excessive but just the result of having done some physical activity. 
In terms of the Btx-A treatment, clinical trials have shown that side effects are rare and predictable. Excessive muscle weakness may be experienced in high dosage and rarely has mild flu-like illness appeared about 7-10 days post injection. The single, controlled dosage used in the proposed study is not expected to result in any adverse reaction. Any pain associated with the injection itself typically subsides within 24 hours.

\section{Benefits}

You will not benefit directly from participating in this study. However, the information gained from your participation will help identify the nature of any physical benefits associated with the treatment, whether they are sustained for a period of time and if there are specific characteristics that indicate which individuals are most likely to experience benefit from the treatment. These findings are valuable for clinical decision making.

\section{Exclusions}

If you have any mobility limiting disorder other than stroke you will be excluded from the study.

\section{Treatment coverage}

The cost of the Btx-A treatment may be covered through your insurance or a request for approval for treatment will be made through the Ministry of Health. Btx-A used for study participants will be covered by the funding provided for this study. You will be eligible to receive the Btx-A treatment whether you chose to participate or not.

\section{Confidentiality}

All information obtained during the course of this study is strictly confidential and your anonymity will be protected at all times. You will be identified by a code based on the date of entry into the study and a project identifier. Data will be stored in locked files and in electronic format in a password protected location with access only to the investigators associated with this study. You will not be identified in any publication, presentation or report.

Voluntary nature of study/Freedom to withdraw or participate

Your participation in this study is voluntary. You may withdraw from this study at any time for any reason and your withdrawal will not affect present or future care.

\section{Withdrawal of subject by principal investigator or co-investigators}

A study investigator may decide to withdraw you from this study if for whatever reason you are unable to report for a scheduled laboratory visit and are unable to reschedule within a reasonable time frame.

\section{Liability}

In the unlikely event that you are injured as a result of the study procedures, health care will be provided to you until resolution of the problem.

By signing this consent form, you do not waive your legal rights nor release the investigators from their legal and professional responsibilities. 


\section{Payment}

We appreciate your involvement in this study. We will cover your travel expenses to the laboratory for testing purposes (mileage or taxi fare). Parking will be provided if needed in front of the School of Rehabilitation Therapy building (Louise D. Acton building).

\section{SUBJECT STATEMENT AND SIGNATURE SECTION:}

I have read and understand the consent form for this study. I have had the purposes, procedures and technical language of this study explained to me. I have been given sufficient time to consider the above information and to seek advice if I chose to do so. I have had the opportunity to ask questions which have been answered to my satisfaction. I am voluntarily signing this form. I will receive a copy of this consent form for my information.

If at any time I have further questions, problems or adverse events, I can contact:

$\begin{array}{llll}\begin{array}{l}\text { Dr. Brenda Brouwer, Co-Principal Investigator } \\ \text { or }\end{array} & \text { at } & 533-6087 \\ \begin{array}{l}\text { Dr. Stephen Bagg, Co-Principal Investigator } \\ \text { or }\end{array} & \text { at } & 544-4210 \\ \begin{array}{l}\text { Dr. David Walker, Dean of the } \\ \text { at }\end{array} & 533-2544\end{array}$

Faculty of Health Sciences

If I have questions regarding my rights as a research subject I can contact

Dr. Albert Clark, Chair, Research Ethics Board at $\quad$ 533-6081

By signing this consent form, I am indicating that I agree to participate in this study.

$\overline{\text { Signature of Subject }} \quad \overline{\text { Date }}$

Signature of Witness Date

\section{STATEMENT OF INVESTIGATOR:}

I, or one of my colleagues, have carefully explained to the subject the nature of the above research study. I certify that, to the best of my knowledge, the subject understands clearly the nature of the study and demands, benefits, and risks involved to participants in this study.

Signature of Principal Investigator

Date 


\section{Appendix C}

\section{Tardieu Scale}

\section{Quality of muscle reaction $(\mathrm{X})$ :}

$0 \quad$ No resistance throughout the course of the passive movement.

1 Slight resistance throughout the course of the passive movement, with no clear catch at a precise angle.

2 Clear catch at a precise angle, interrupting the passive movement, followed by release.

3 Fatigable clonus ( $<10$ seconds when maintaining pressure) occurring at a precise angle.

$4 \quad$ Infatigable clonus ( $>10$ seconds when maintaining pressure) occurring at a precise angle.

\section{Velocity of stretch:}

$\mathrm{R} 1$ : As fast as possible (faster than the rate of the natural drop of the limb segment under gravity).

R2: As slow as possible (minimizing stretch reflex).

Angle of muscle reaction (Spasticity angle): Difference between R1 and R2; measured relative to the position of minimal stretch of the muscle (corresponding to angle 0 ) for all joints except hip, where it is relative to the resting anatomic position.

Grading is always performed in a constant position of the body for a given limb. For each muscle group, reaction to stretch is rated at a specified stretch velocity with 2 parameters, $\mathrm{X}$ and $\mathrm{Y}$.

(Gracies et al., 2000) 


\section{Appendix D}

BTX-A dosages and injection sites of individual subjects

\begin{tabular}{ccccc}
\hline Subject & Gastrocnemius & Soleus & $\begin{array}{c}\text { Tibialis } \\
\text { Posterior }\end{array}$ & $\begin{array}{c}\text { Flexor } \\
\text { Digitorum } \\
\text { Longus }\end{array}$ \\
\hline 1 & 125 & 100 & 75 & --- \\
2 & 125 & 100 & 75 & --- \\
3 & 100 & 100 & 100 & 100 \\
4 & 125 & 100 & 100 & 75 \\
5 & 100 & 100 & 100 & 100 \\
6 & 100 & 100 & 100 & --- \\
7 & 100 & 100 & 100 & -- \\
\hline
\end{tabular}




\section{Appendix E}

\section{Botulinum Toxin A (BTX-A) standard clinical dosage guidelines}

\begin{tabular}{|c|c|c|c|}
\hline \multicolumn{4}{|c|}{ Suggested Pediatric Dosing* } \\
\hline Clinical pattern & $\begin{array}{c}\text { Potential muscles } \\
\text { involved }\end{array}$ & $\begin{array}{l}\text { BOTOX dose* } \\
\text { units/kg }\end{array}$ & $\begin{array}{c}\text { Number of } \\
\text { injection sites }\end{array}$ \\
\hline \multirow[t]{6}{*}{ Equinovarus foot } & $\begin{array}{c}\text { Gastrocnemius } \\
\text { medial/lateral }\end{array}$ & $3-6$ & $1-4$ \\
\hline & Soleus & $2-3$ & $1-2$ \\
\hline & Tibialis posterior & $1-2$ & 1 \\
\hline & Tibialis anterior & $1-3$ & 1 \\
\hline & $\begin{array}{l}\text { Flexor digitorum } \\
\text { brevis/longus }\end{array}$ & $1-2$ & 1 \\
\hline & $\begin{array}{l}\text { Flexor hallucis } \\
\text { longus }\end{array}$ & $1-2$ & 1 \\
\hline Striatal toe & $\begin{array}{l}\text { Extensor hallucis } \\
\text { longus }\end{array}$ & $1-2$ & 1 \\
\hline
\end{tabular}

*Adult dosing recommendations should be substituted for children heavier than $60 \mathrm{~kg}$

(modified from http://www.wemove.org/spa/) 


\section{Appendix F}

Angular difference (degrees) between baseline 1 (B1) and baseline 2 (B2) kinematic measures, from the affected (A) and unaffected (UA) sides for each subject 
Kinematic differences (degrees) between B1 and B2 at the ankle, knee and hip

\begin{tabular}{|c|c|c|c|c|c|c|c|}
\hline \multirow{2}{*}{ Subject } & \multirow{2}{*}{ Side } & \multicolumn{2}{|c|}{ Ankle } & \multicolumn{2}{|c|}{ Knee } & \multicolumn{2}{|c|}{ Hip } \\
\hline & & MaxDF & MaxPF & MaxFlex & MaxExt & MaxFlex & MaxExt \\
\hline 1 & A & \multicolumn{6}{|c|}{ No Baseline 1 Measures } \\
\hline \multirow{2}{*}{2} & $\mathbf{A}$ & 2.1 & 2.4 & 1.7 & 0.6 & 1.8 & 3.1 \\
\hline & UA & 0.3 & 4.2 & 2.0 & 4.0 & 4.4 & 3.1 \\
\hline \multirow{2}{*}{3} & $\mathbf{A}$ & 0.1 & 0.1 & 3.0 & 1.8 & 0.1 & 0.5 \\
\hline & UA & 1.3 & 2.4 & 3.9 & 3.1 & 2.3 & 3.3 \\
\hline \multirow{2}{*}{4} & $\mathbf{A}$ & 2.3 & 2.0 & 1.8 & 4.9 & 5.1 & 0.5 \\
\hline & UA & 3.1 & 0.1 & 3.8 & 0.7 & 4.9 & 6.0 \\
\hline \multirow{2}{*}{5} & $\mathbf{A}$ & 1.3 & 1.0 & 0.1 & 0.4 & 5.7 & 2.8 \\
\hline & UA & 2.1 & 0.6 & 8.2 & 1.9 & 3.4 & 4.6 \\
\hline \multirow{2}{*}{6} & $\mathbf{A}$ & 2.1 & 1.2 & 3.2 & 0.3 & 1.7 & 0.6 \\
\hline & UA & 0.8 & 1.3 & 2.9 & 2.2 & 2.5 & 3.5 \\
\hline \multirow{2}{*}{7} & $\mathbf{A}$ & 1.1 & 1.9 & 5.5 & 2.7 & 1.9 & 1.6 \\
\hline & $\mathbf{U A}$ & 3.5 & 2.1 & 7.5 & 3.7 & 0.7 & 2.7 \\
\hline
\end{tabular}

$\mathrm{A}=$ Affected side; $\mathrm{UA}=\mathrm{Unaffected}$ side; $\mathrm{MaxDF}=\mathrm{Maximum}$ dorsiflexion angle; MaxPF $=$ Maximum plantarflexion angle MaxFlex = Maximum flexion angle; MaxExt $=$ Maximum extension angle 


\section{Appendix G}

Kinematic and kinetic profiles of individual subjects during stance at baseline (light grey), two weeks (T1, red), six weeks (T2, black) and ten weeks (T3, blue) post BTX$A$ injection. The light shaded grey area represents standard deviation of baseline 1 and baseline 2 


\section{Subject 1 Kinematics $\left({ }^{\circ}\right)$ \\ Affected side: Left}
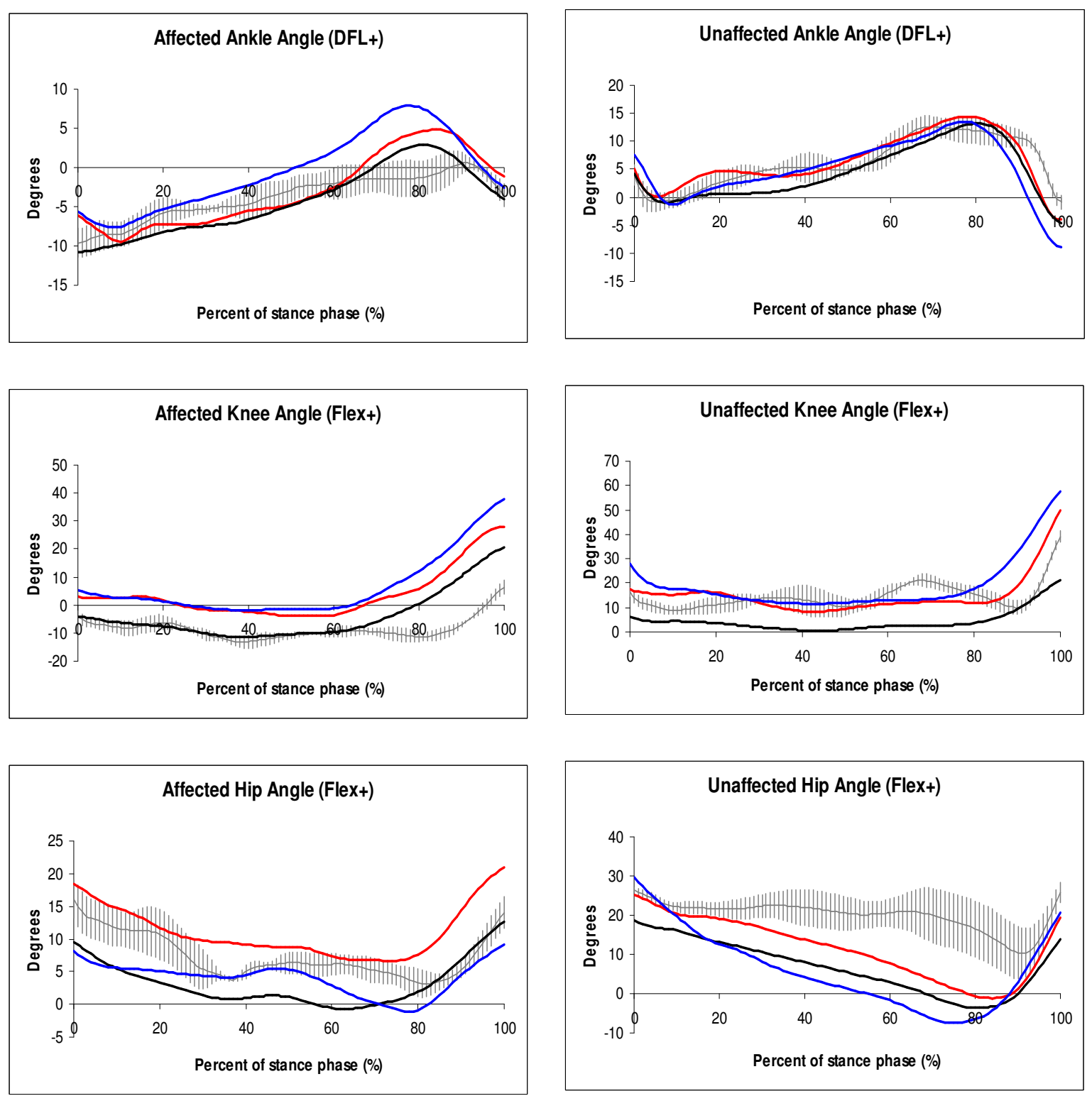


\section{Affected side: Left}
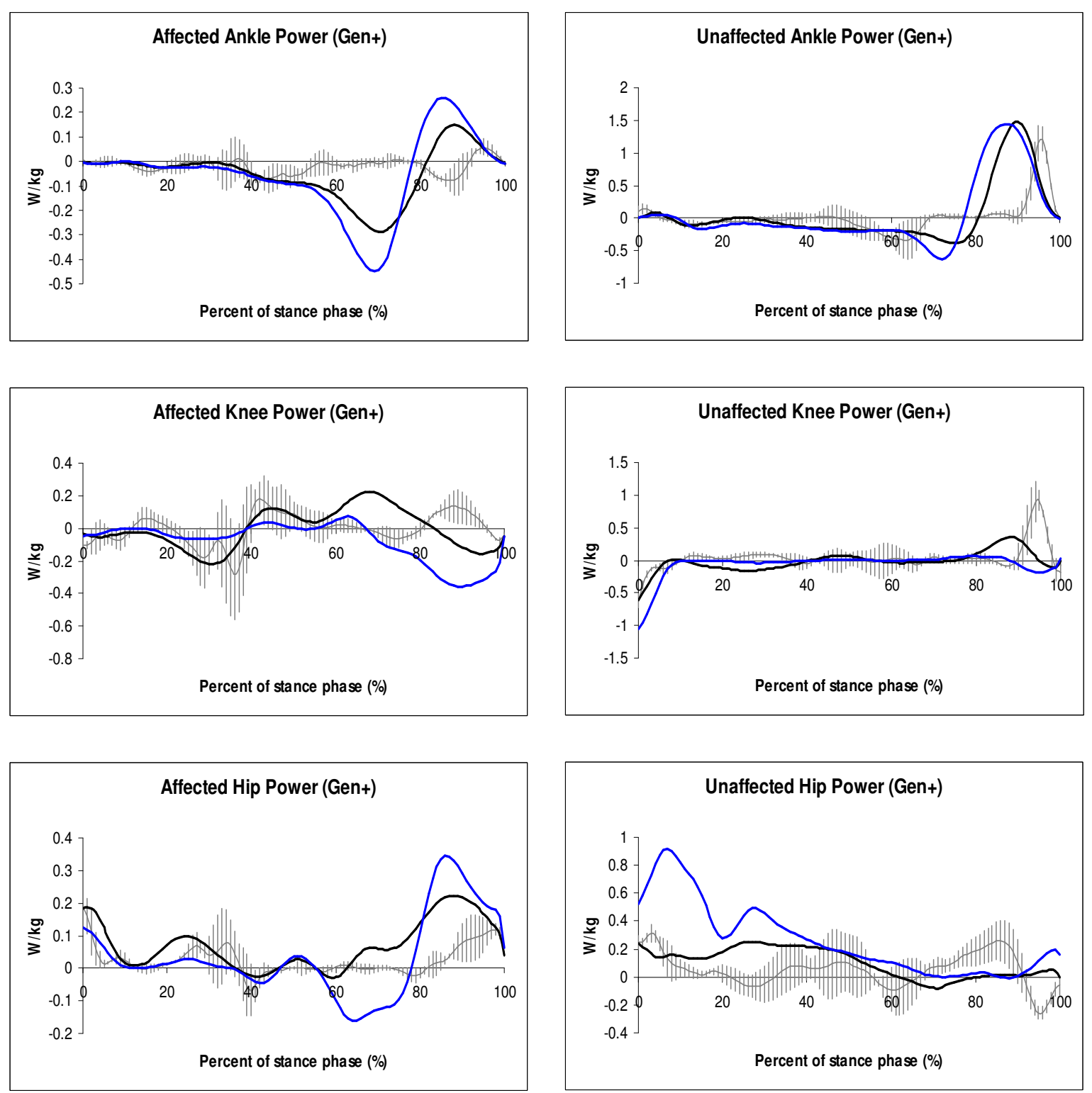


\section{Subject 2 Kinematics $\left({ }^{\circ}\right)$ Affected side: Right}
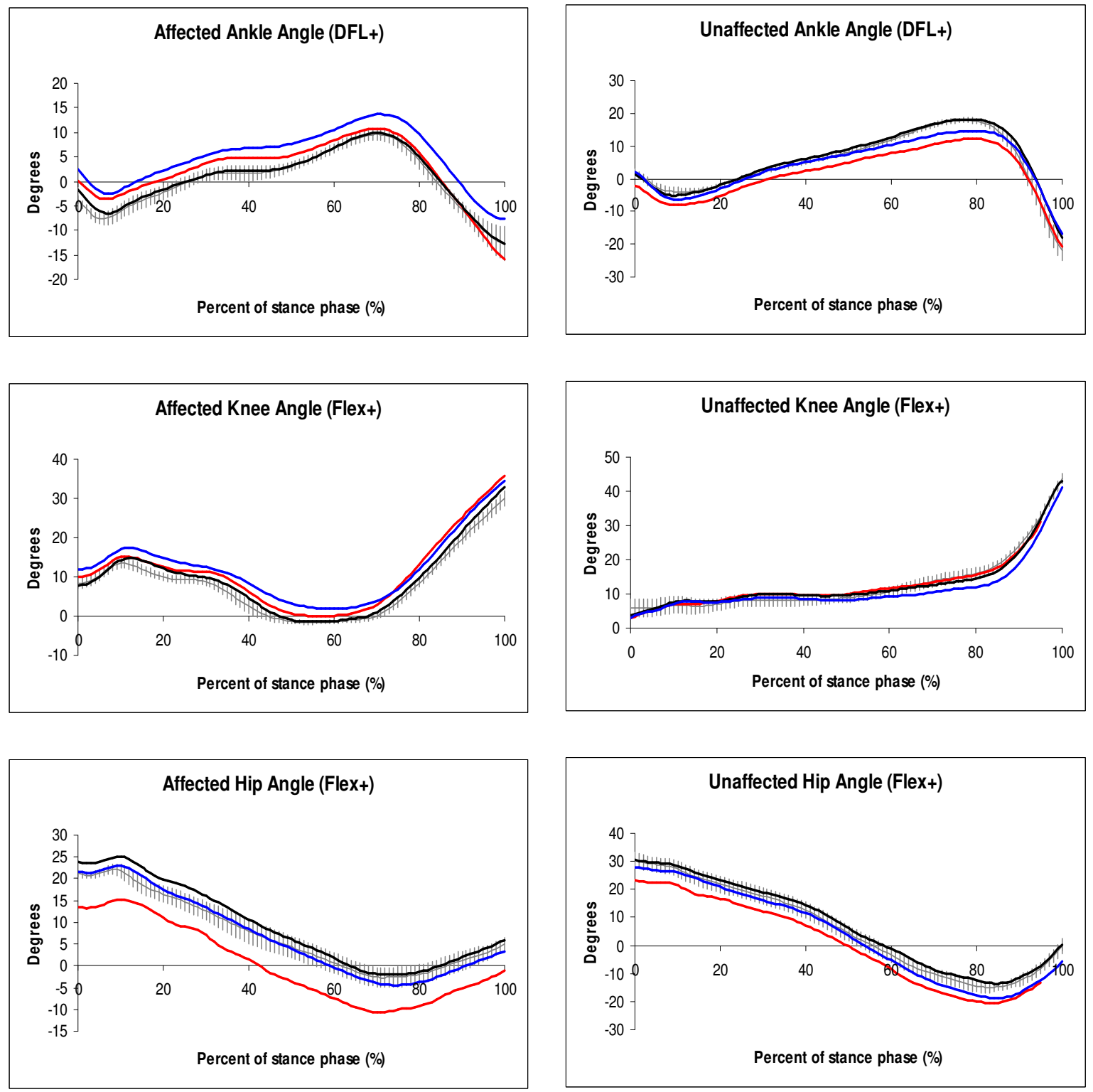
Subject 2 Powers (W/kg)

Affected side: Right
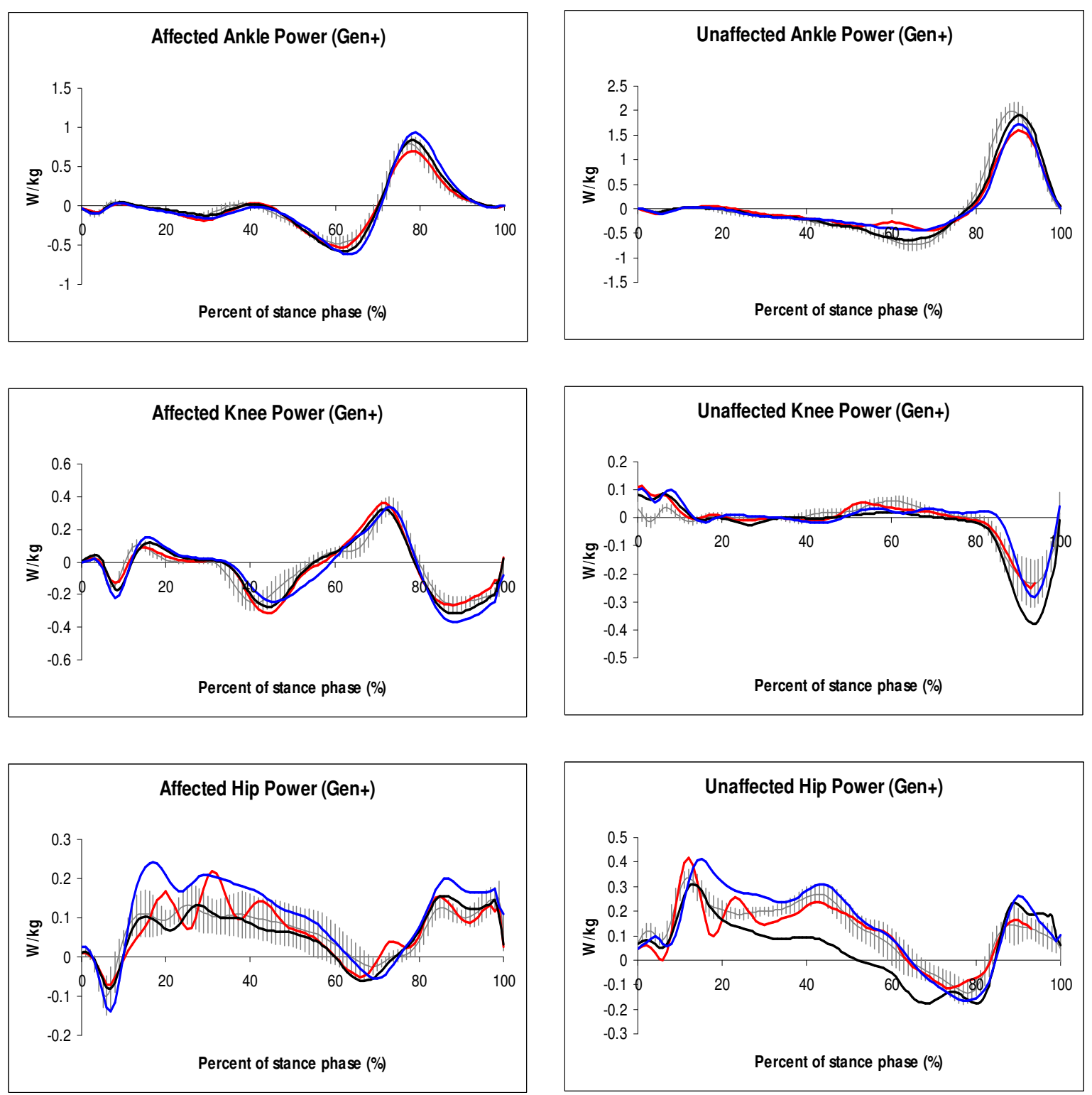


\section{Subject 3 Kinematics $\left({ }^{\circ}\right)$ \\ Affected side: Left}
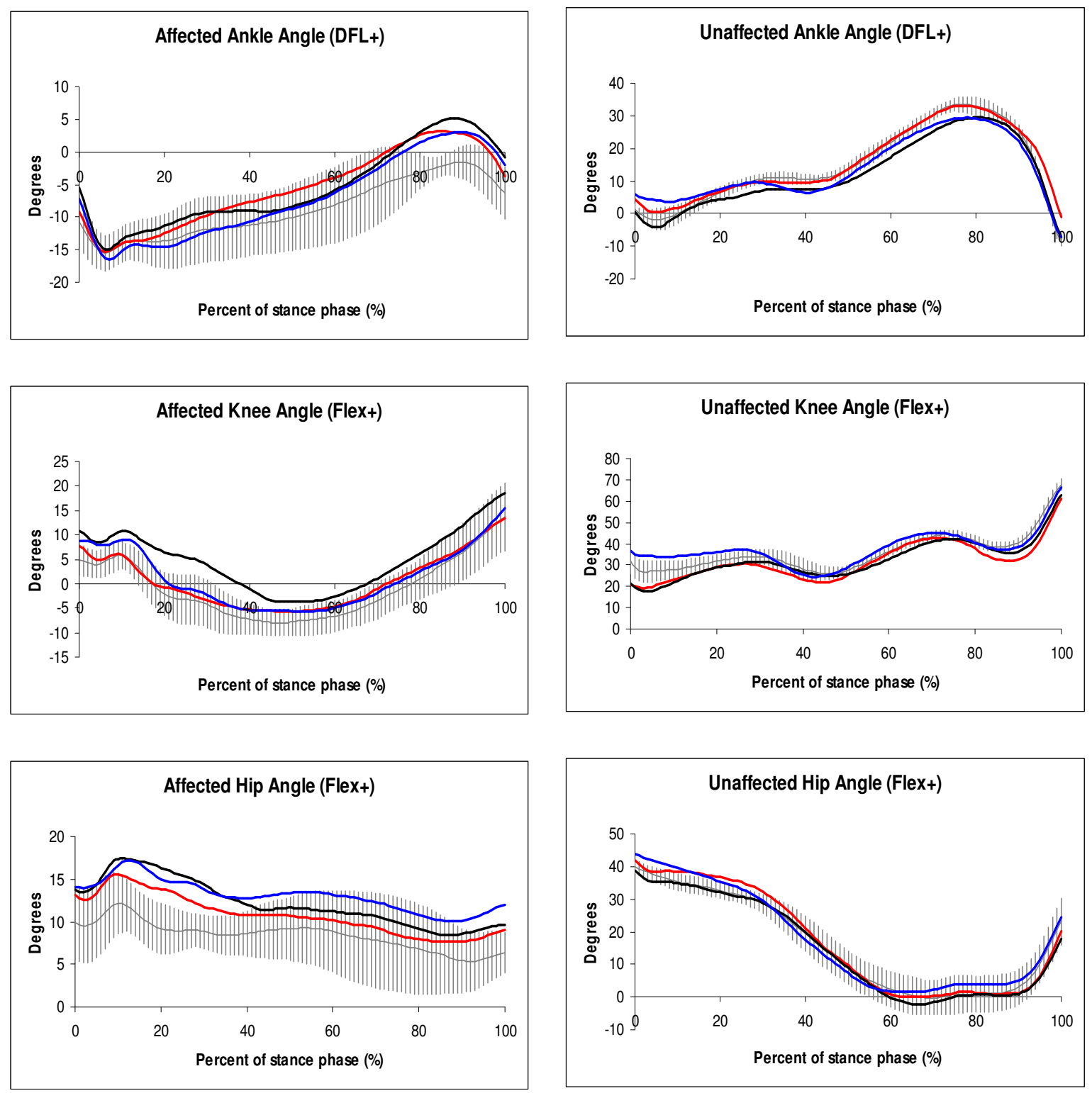
Subject 3 Powers ( $W /$ kg)

\section{Affected side: Left}
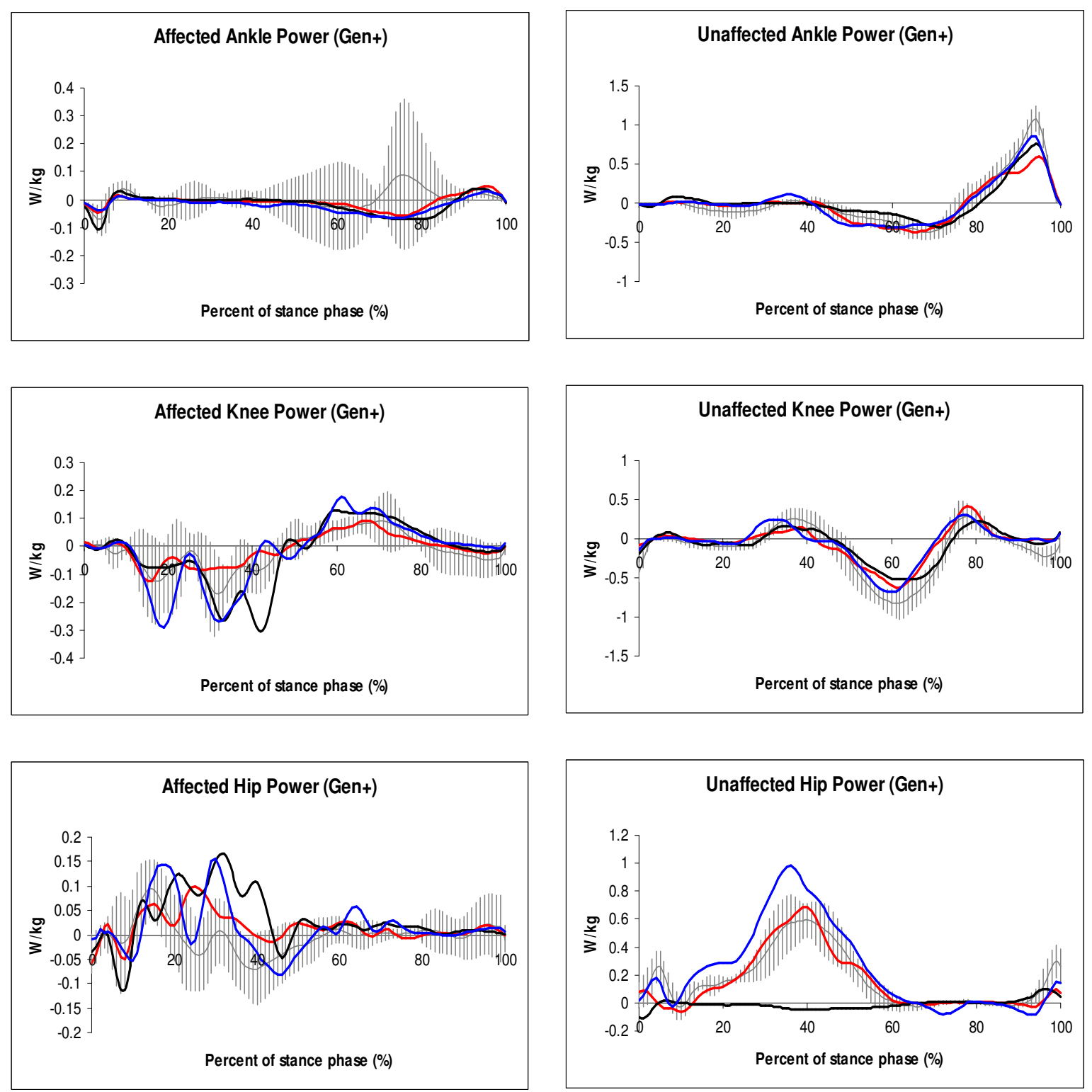


\section{Subject 4 Kinematics $\left({ }^{\circ}\right)$ Affected side: Right}
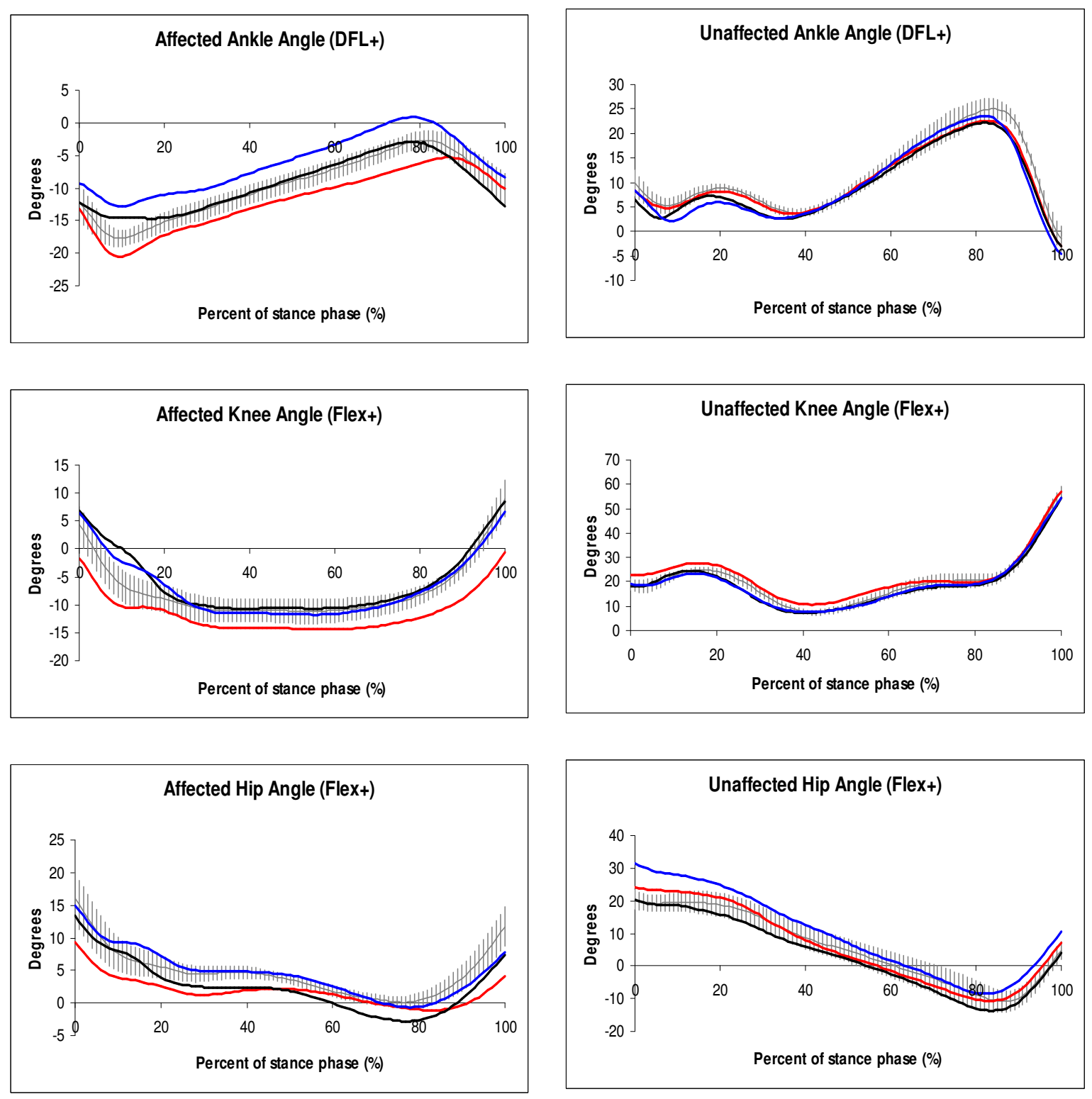

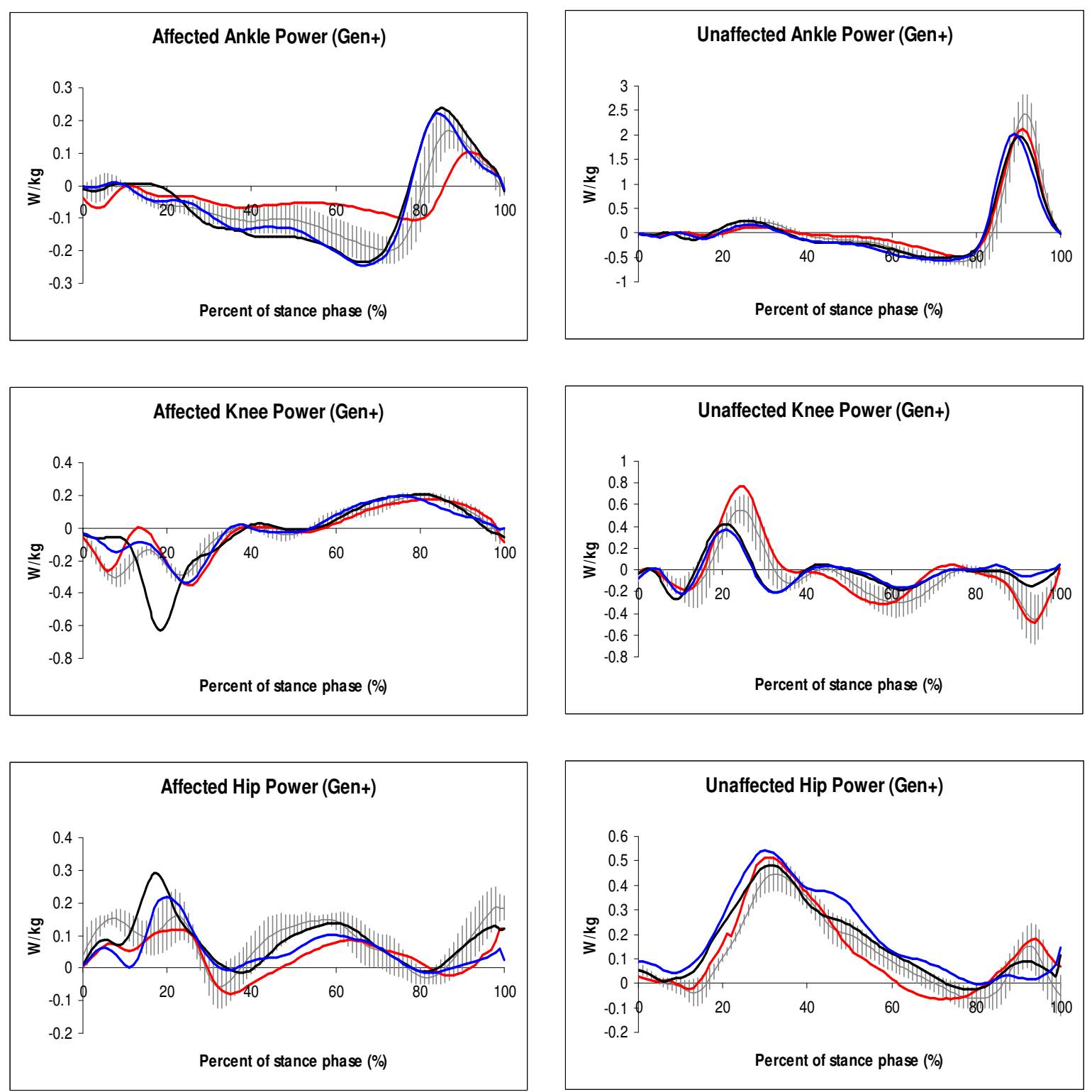


\section{Subject 5 Kinematics $\left({ }^{\circ}\right)$ Affected side: Right}
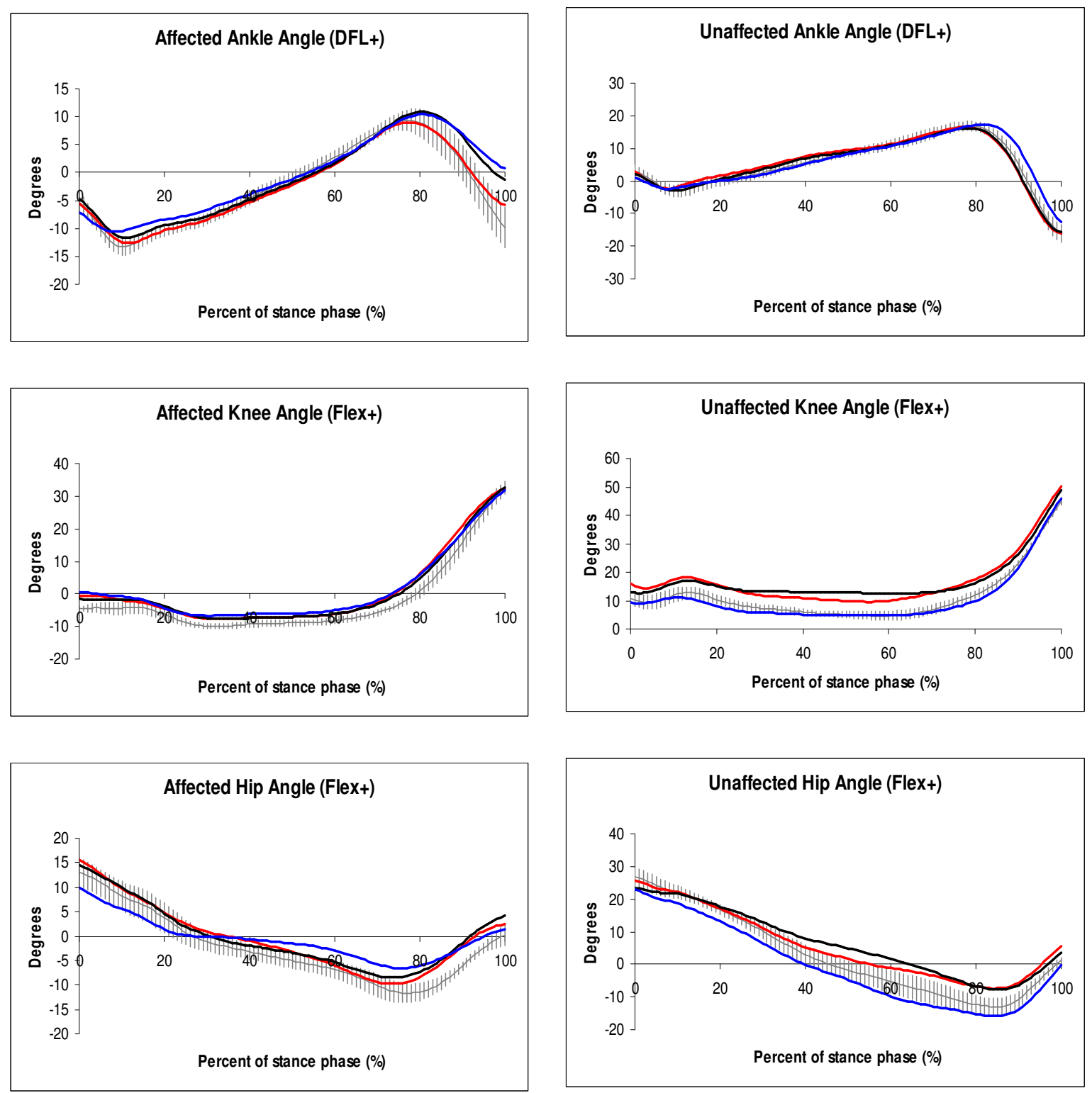
Subject 5 Powers (W/kg)

Affected side: Right
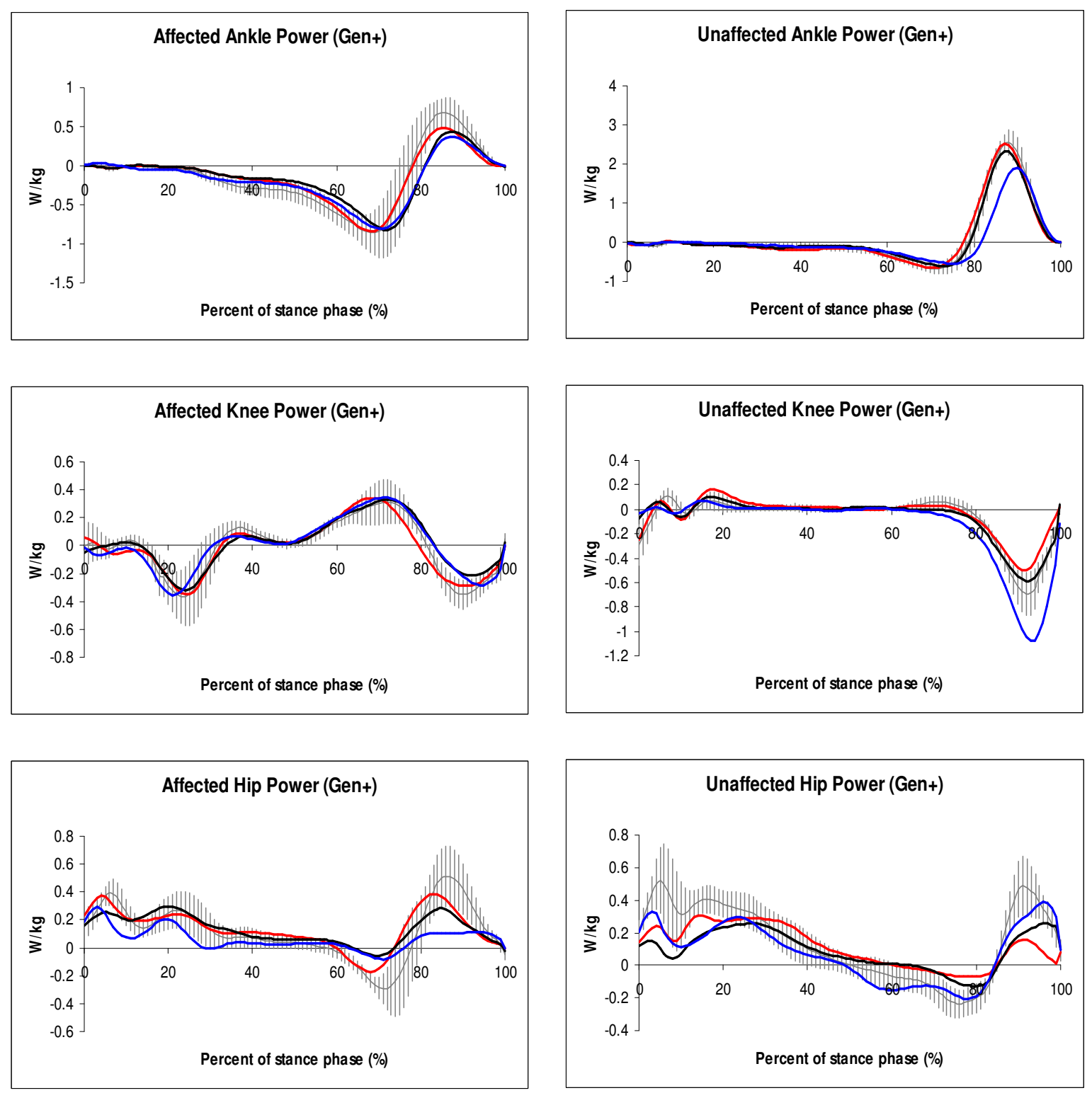


\section{Subject 6 Kinematics $\left({ }^{\circ}\right)$ \\ Affected side: Right}
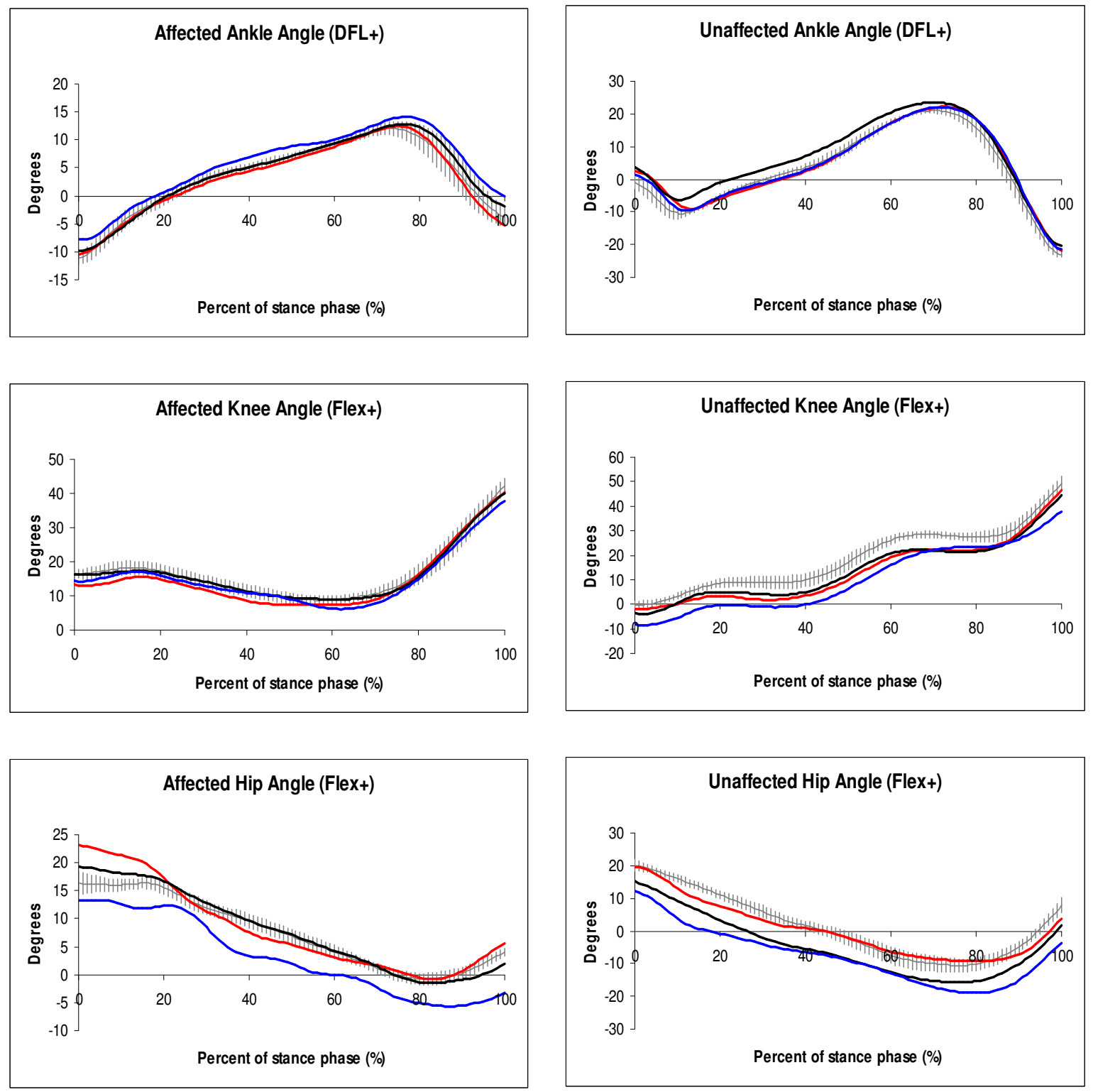

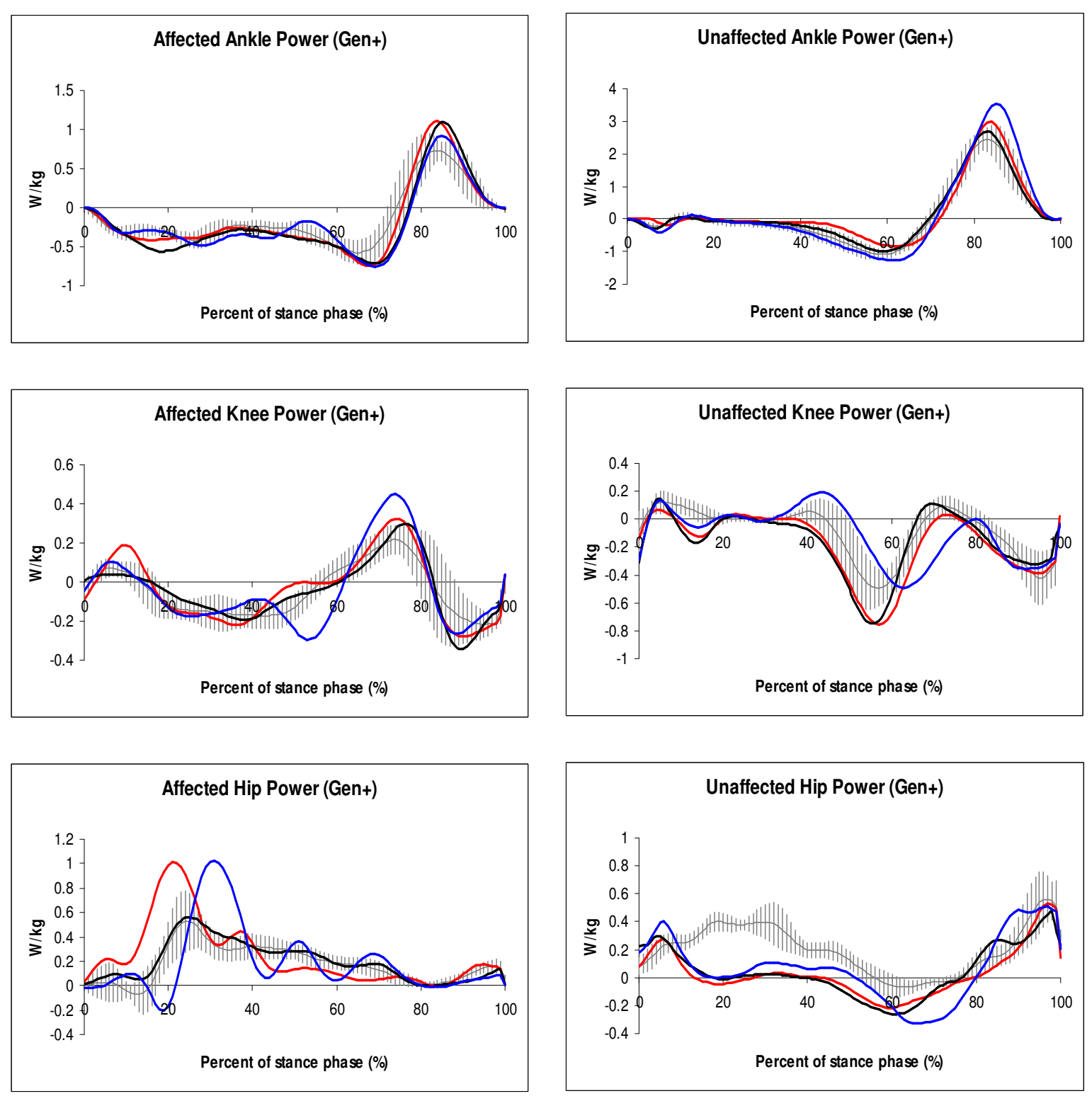


\section{Subject 7 Kinematics $\left({ }^{\circ}\right)$ Affected side: Left}
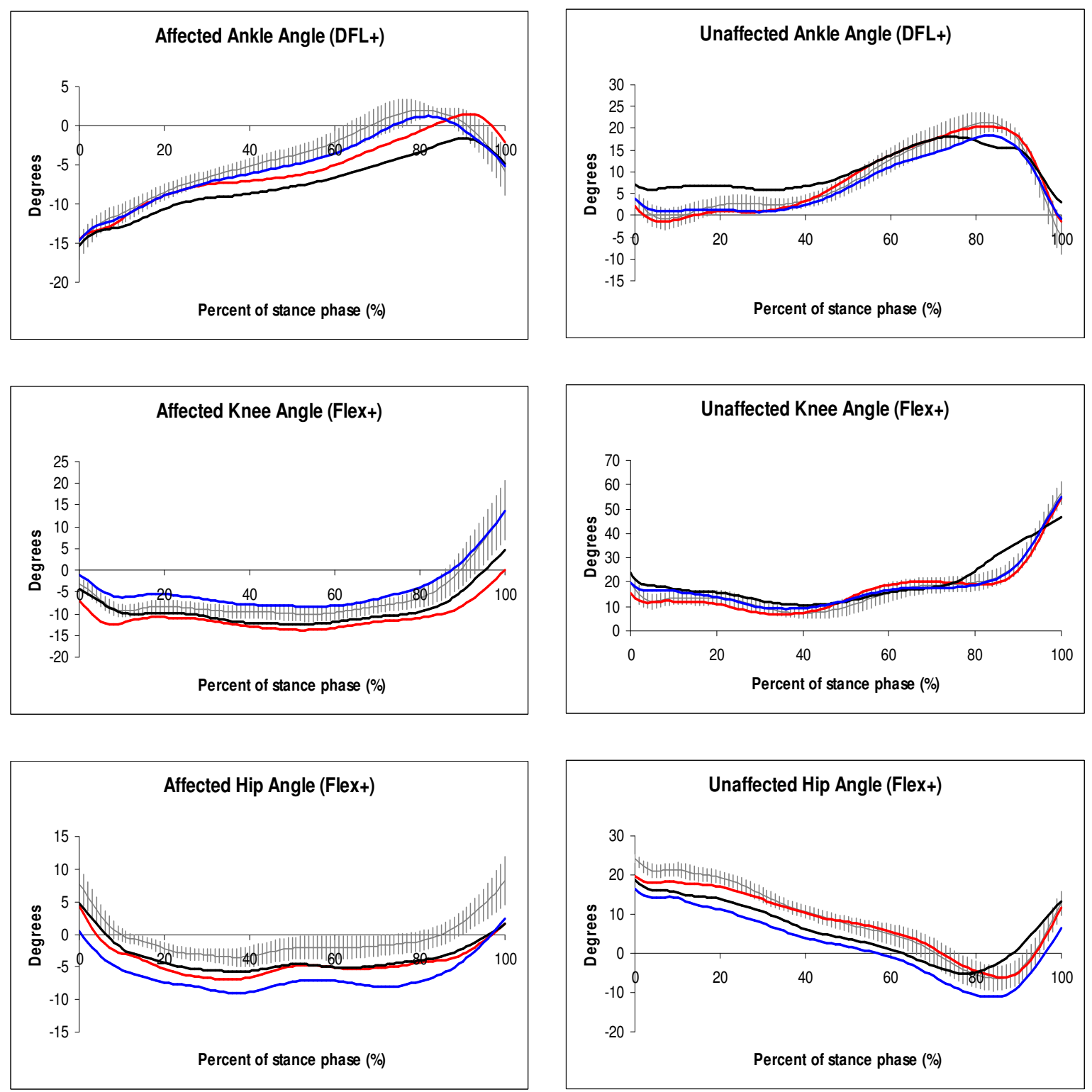


\section{Subject 7 Powers (W/kg) \\ Affected side: Left}
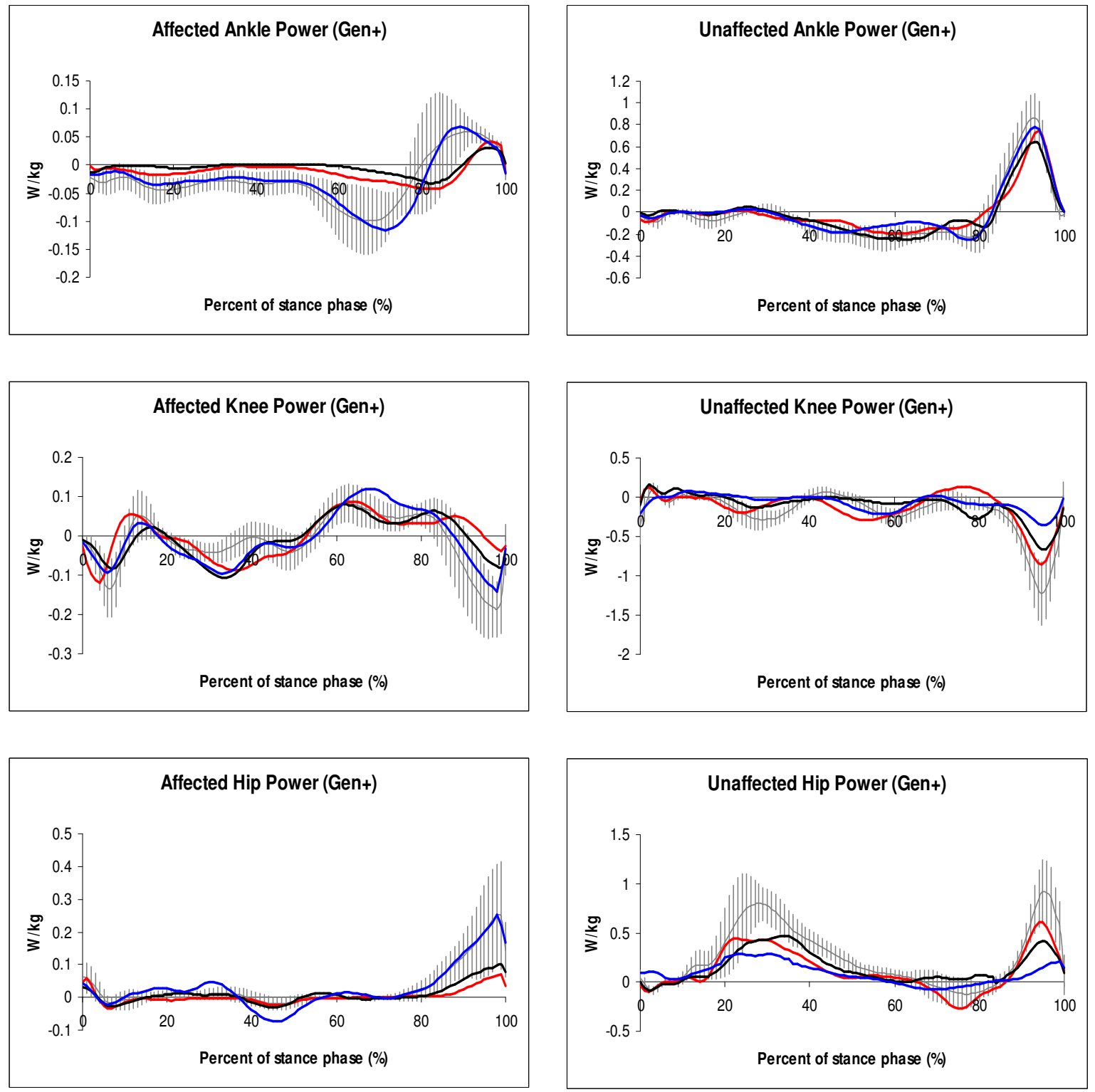